\title{
Deep WFPC2 and Ground-based Imaging of a Complete Sample of 3C Quasars and Galaxies ${ }^{1}$
}

\author{
Susan E. Ridgway ${ }^{2,3,4}$ and Alan Stockton ${ }^{3,4}$ \\ Institute for Astronomy, University of Hawaii, 2680 Woodlawn Drive, Honolulu, Hawaii \\ 96822 \\ Electronic mail: s.ridgway1@physics.oxford.ac.uk, stockton@uhifa.ifa.hawaii.edu
}

\begin{abstract}
We present the results of an HST and ground-based imaging study of a complete $3 \mathrm{C}$ sample of $z \sim 1$ sources, comprising 5 quasars and 5 radio galaxies. We have observed all of the sample in essentially line-free bands at rest-frame $0.33 \mu \mathrm{m}$ with WFPC2 and in rest-frame $1 \mu \mathrm{m}$ images from the ground; we have also observed most of the sample in narrow-band filters centered on [O II]. We resolve continuum structure around all of our quasars in the high-resolution WFPC2 images, and in four of the five ground-based $K^{\prime}$ images. All of the quasars have some optical continuum structure that is aligned with the radio axis. In at least 3 of these cases, some of this optical structure is directly coincident with a portion of the radio structure, including optical counterparts to radio jets in 3C 212 and $3 \mathrm{C} 245$ and an optical counterpart to a radio lobe in $3 \mathrm{C} 2$. These are most likely due to optical synchrotron radiation, and the radio and optical spectral indices in the northern lobe of $3 \mathrm{C} 2$ are consistent with this interpretation.

The fact that we see a beamed optical synchrotron component in the quasars but not in the radio galaxies complicates both the magnitude and the alignment
\end{abstract}

\footnotetext{
${ }^{1}$ Based on observations made with the NASA/ESA Hubble Space Telescope, obtained at the Space Telescope Science Institute, which is operated by the Association of Universities for Research in Astronomy, Inc., under NASA contract NAS 5-26555.

${ }^{2}$ Current address: University of Oxford, Department of Physics, Nuclear and Astrophysics Laboratory, Keble Road, Oxford, OX1 3RH.

${ }^{3}$ Visiting Astronomer, W.M. Keck Observatory, jointly operated by the California Institute of Technology and the University of California.

${ }^{4}$ Visiting Astronomer, Canada-France-Hawaii Telescope, operated by the National Research Council of Canada, the Centre National de la Recherche Scientifique de France, and the University of Hawaii.
} 
comparisons. Nonetheless, the total optical and $K^{\prime}$ flux densities of the quasar hosts are consistent with those of the radio galaxies within the observed dispersion in our sample. The distributions of $K^{\prime}$ flux densities of both radio galaxies and quasar hosts exhibit similar mean and dispersion to that found for other radio galaxies at this redshift, and the average host galaxy luminosity is equivalent to, or a little fainter than, $L^{*}$. The formal determination of the alignment in the optical and infrared in the two subsamples yields no significant difference between the radio galaxy and quasar subsamples, and the quasars 3C 196 and 3C 336 have aligned continuum and emission-line structure that is probably not due to beamed optical synchrotron emission.

Very blue and/or edge-brightened structures are present in some objects within the probable quasar opening angle; these are possibly the result of illumination effects from the active nucleus, i.e., scattered quasar light or photoionization. In $3 \mathrm{C} 212$, we see an optical object that lies $3^{\prime \prime}$ beyond the radio lobe, but which looks morphologically quite similar to the radio lobe itself. This object is bright in the infrared and has a steep spectral gradient along its length. A striking, semi-circular arc seen associated with 3C 280 may possibly be a tidal tail from a companion, enhanced in brightness by scattering or photoionization.

In the near-infrared, most of the radio galaxies have elliptical morphologies with profiles that are well-fit by de Vaucouleurs $\mathrm{r}^{\frac{1}{4}}$-laws and colors that are consistent with an old stellar population. All components around the quasars have optical-infrared colors that are redder than or similar to the colors of their respective nuclei; this is more consistent with a stellar origin for the emission than with a dominant scattering contribution.

From the correspondence between the total magnitudes in the galaxies and quasars and the detection of aligned components in the quasars, we conclude that this study provides general support for the unification of FR II radio galaxies and quasars. Some of the objects in the sample (e.g, 3C 212) have properties that may be difficult to explain with our current understanding of the nature of FR II radio sources and the alignment effect.

\section{Introduction}

Powerful radio sources, and the objects associated with them, provide probes of the Universe from $z \sim 1$ up to the highest redshifts at which discrete objects have been detected. Over the past decade, observations of radio sources at high redshift have focussed primarily on the galaxies, since the extended hosts of quasars at those redshifts are much 
more difficult to observe. We will present here the results of a systematic imaging survey of a complete sample of $z \sim 1$ radio galaxies and quasars; first we will briefly review the background and motivation for this work.

\subsection{High Redshift Radio Galaxies}

Observations of high- $z$ radio galaxies have been reviewed by McCarthy (1993); here we summarize and update the aspects most relevant to our investigation. Radio galaxies at $z \gtrsim 0.8$ often have both distorted, multi-component optical continuum structures and associated emission-line regions extending many tens of $\mathrm{kpc}$. Both the emission-line and the continuum shapes tend to be elongated along the direction of the radio axis (Chambers, Miley, \& van Breugel 1987; McCarthy et al. 1987), whereas similar alignments have been observed only rarely in the lower-redshift $(z<0.6)$ 3C sample (Baum \& Heckman 1989; McCarthy \& van Breugel 1989).

This "alignment effect" also seems to be a function of wavelength; near-infrared morphologies of $z \sim 1$ radio galaxies are rounder and the strength of the aligned component is generally less, though in many cases still significant (Rigler et al. 1992; Dunlop \& Peacock 1993). It cannot be assumed, of course, that the differences between the high and low redshift galaxies arise solely from evolutionary effects. Optical imaging of high redshift galaxies corresponds to the rest-frame ultraviolet, which is observable only with great difficulty from the ground for their low- $z$ counterparts. Another difference between the high- $z$ and low- $z$ samples is that the radio power of the low-redshift sources in a flux-limited sample is systematically lower than that of the high- $z$ objects. Observations of morphologies of samples of low radio power galaxies at high redshift have found no evidence for significant alignment, indicating a radio power dependence to the alignment effect (Dunlop \& Peacock 1993; Eales et al. 1997).

A simple explanation for this correlation of optical and infrared morphologies with the radio axis remains elusive, and observations probably rule out any single cause. Early attempts were made to interpret the morphologies of high-redshift radio galaxies in terms of interactions (e.g., Djorgovski et al. 1987), which seemed an attractive and natural way to explain the observed distorted morphologies and close companions. Such explanations were largely abandoned with the discovery of the alignment effect, because it seemed unlikely that the interaction axis and the radio axis would be naturally correlated with each other (but see West 1994).

Currently, two processes are generally considered as the most probable causes of the 
alignment effect: jet-induced star formation, and the scattering of light from an obscured quasar. High polarizations $(\sim 10 \%)$ have been confirmed in 3C 277.3, 3C 324, 3C 256, 3C 265 and in a number of other radio galaxies (Cimatti et al. 1993, 1996; Dey et al. 1996). Direct detections of broad $\mathrm{Mg}$ II lines in polarized light from the aligned continuum in 3 high- $z$ radio galaxies (di Serego Alighieri et al. 1994), and detection of broad components to Mg II $\lambda 2798$ and C III] $\lambda 1909$ in deep spectra of 3C 324 (Dickinson, Dey \& Spinrad 1995) make it very likely that scattered quasar radiation is a significant component of the aligned optical light in at least these galaxies (Fabian 1989; Cimatti et al. 1993; di Serego Alighieri et al. 1994).

However, high-resolution HST WFPC2 imaging of $z \sim 1$ radio galaxies generally has not revealed a "scattering cone" morphology as is seen in low redshift AGN such as NGC 1068; the alignment comes in many cases from discrete lumps that are closely confined to the track of the radio jet (Longair et al. 1995; Dickinson et al. 1995; Best, Longair \& Röttgering 1996). This morphological evidence tends to favor the suggestion that the aligned emission is dominated by star formation induced by the radio jet (Chambers et al. 1987; Begelman \& Cioffi 1989; de Young 1989; Rees 1989; Daly 1990). Longair et al. (1995) and Best et al. (1996) find a variety of morphologies in their sample of $8 z \sim 1$ galaxies, and, though most exhibit some degree of alignment, the nature of the correspondence with the radio structure varies from source to source. The smaller scale radio sources such as $3 \mathrm{C} 324$ and $3 \mathrm{C} 368$ have structures aligned close to the radio axis, while the larger scale radio sources tend to have material that appears more relaxed, exhibiting less alignment. Best et al. speculate that this is an age effect: if the very closely aligned structures seen in the smaller, younger radio sources are the result of radio-jet-induced star formation, this activity would cease after the radio lobes have passed outside of the host galaxy, allowing the stars formed to relax into the compact morphologies observed in the larger, and presumably older radio sources.

There is so far no conclusive evidence for a case in which stellar emission in likely to dominate an aligned component, in spite of the early claim of Chambers \& McCarthy (1990) for possible stellar absorption features in summed spectra of aligned regions in two different radio galaxies. The best example of an object believed to be dominated by stars formed by a radio jet is Minkowski's Object, a blue clump of line-emitting material in the path of the jet from a $z \sim 0.018$ radio galaxy. Its broad-band colors, line ratios and emission-line morphology make jet-induced star formation the most likely interpretation (Brodie, Bowyer, \& McCarthy 1985; van Breugel et al. 1985; Hansen, Nørgaard-Nielsen, \& Jørgensen 1987) but even in this low-redshift case it is difficult to get conclusive proof of the existence of a young stellar population. 
Other possible contributors to the aligned UV continuum in these galaxies are thermal emission from a hot plasma, optical synchrotron radiation, and inverse Compton scattering of microwave background photons by relativistic electrons (Chambers et al. 1988; Daly $1992 a, b)$. Some of these mechanisms require that the optical morphology directly trace the radio structure: such a correspondence may be present in some smaller scale radio objects such as 4C 41.17 (Miley et al. 1992), but these mechanisms cannot account for the many cases in which there is no strong correlation between the radio and optical morphologies. The aligned components of the blue, closely aligned $z \sim 1$ galaxy 3C 368, originally thought to be dominated by scattered quasar light, seem low in polarization in recent imaging polarimetry and spectropolarimetry (Dey et al. 1997). These components have Balmer-line-to-continuum ratios that indicate the thermal nebular continuum from the ionized gas actually dominates the near-UV aligned continuum radiation (Dickson et al. 1995; Stockton, Ridgway \& Kellogg 1996).

\subsection{QSO Host Galaxies}

Far less is known about the hosts of high redshift quasars than about radio galaxies. Until recently, studies of QSO host galaxies have concentrated primarily on the low redshift range. Evidence has accumulated that QSO activity might be triggered by interactions or mergers: the extensive ground-based work on these $z<1$ QSOs has shown a significant fraction of them to have asymmetries, distortions, tidal features, and a tendency to have close companions. Their host galaxies have colors bluer than do normal ellipticals; such colors would be consistent with interaction-triggered star formation (Heckman 1990; Stockton 1990 and references therein). In the past few years, several groups have studied radio-loud and radio-quiet QSOs at $z=2$ to 3 (Heckman et al. 1991; Lehnert et al. 1992; Hutchings et al. 1994b; Lowenthal et al. 1995; Arextaga et al. 1995). They have been able to resolve extensions around a reasonable percentage of the high- $z$ QSOs they have studied in optical and/or the near-infrared $(\sim 50 \%)$; these groups find similar results in that the extended portion contributes $\sim 20 \%$ of the total quasar flux at observed optical wavelengths. Lehnert et al. (1992) found the extended quasar light to have redder optical-infrared colors than the quasar itself. This result is more consistent with the extensions coming from a stellar host galaxy than from scattered nuclear emission. They also found that the 5 quasars in their sample have total host $K$ magnitudes significantly brighter than those of radio galaxies in a comparable redshift range. In the optical studies of the same sample, Heckman et al. (1991) saw no evidence for alignment of the quasar extensions with the radio axis. These two results seem to pose difficulties for theories in which radio galaxies and quasars differ solely in orientation (Barthel 1989); radio galaxies at these redshifts are 
quite aligned in the UV. Recently, however, Bremer (1997) has found that the $z=0.734$ quasar 3C 254 has continuum and emission-line morphologies that are well aligned with the radio structure.

Of course, the study of QSO host-galaxy properties is complicated by the bright nucleus which contaminates or swamps any extended component that underlies the seeing disk. For this reason, the Hubble Space Telescope (HST) is well-suited to the study of QSO host galaxies, and even more so for these high redshift objects in which (at $z=1$ ) $\sim 6 \mathrm{kpc}$ of physical scale ${ }^{5}$ would be hidden under a typical $1^{\prime \prime}$-diameter groundbased seeing disk. A number of recent WFPC2 imaging studies of QSO host galaxies at low redshift have been made. These include the major GTO survey of $z \sim 0.2$ QSOs of Bahcall et al. (1994, 1995a, $1995 b, 1996,1997)$, a survey of 4 QSOs by Disney et al. (1995), and several low- $z$ QSOs studied by Hutchings et al. (1994a). Early claims of discrepancies in host-galaxy magnitudes between the results of previous ground-based imaging and the HST images (Bahcall et al. 1994, 1995a) now seem to have been resolved (McLeod \& Rieke 1995; Bahcall et al. 1997), and the higher resolution of the HST images allows details of the galaxy morphology to be determined. QSOs in general are found to have a fairly wide range of host galaxies, ranging from apparently normal ellipticals and spirals, to obviously interacting systems. Radio-loud quasars are, as expected, usually found in elliptical host galaxies, but (contrary to general expectation) radio-quiet QSOs seem also to be predominantly in elliptical host galaxies.

\subsection{Unified Models}

The optical spectra and extended radio properties of radio galaxies and quasars have many similarities, inspiring attempts to explain the differences between the two classes as due primarily to orientation (Scheuer 1987; Barthel 1989). In this view, FR II radio galaxies and quasars are drawn from the same population; the non-thermal optical continuum and broad spectral features seen in the quasars are obscured in the radio galaxies by a dusty torus oriented perpendicularly to the radio jet. That radio sources have strongly beamed, relativistic jets is well-established from the observed jet asymmetries, apparent superluminal motion, and depolarization aymmetries between the jet and counter-jet-side radio lobes of quasars (Laing 1988; Garrington et al. 1988; Ghisellini et al. 1993). Orientation must play a part in how we view and classify such beamed objects.

Unified hypotheses of AGNs in general have been reviewed recently by Antonucci (1993) and of radio-loud AGNs in particular by Urry \& Padovani (1995). Spectrophotometric

\footnotetext{
${ }^{5} \mathrm{H}_{\mathrm{o}}=75 \mathrm{~km} \mathrm{sec}^{-1} \mathrm{Mpc}^{-1}, \mathrm{q}_{\mathrm{o}}=1 / 2$ throughout this paper.
} 
studies of $3 \mathrm{C} 234$ and seven other radio galaxies revealed obscured broad-line regions (Antonucci \& Barvainis 1990; see review by Antonucci 1993). These studies, the recent probable discovery of broad Mg II in emission in the very nearby powerful FR II galaxy Cygnus A (Antonucci et al. 1994), and detections of broad $\mathrm{Pa}-\alpha$ from some narrow-line radio galaxies (Hill et al. 1996), provide direct support for this unification of radio galaxies and quasars. Another way to test the unification hypothesis for quasars and narrow-line radio galaxies is to compare properties that should be isotropic in matched, complete, and unbiased samples of radio galaxies and quasars selected on the basis of some other supposedly isotropic property, such as extended radio emission. As summarized by Urry \& Padovani (1995), a number of such tests can and have been made, with results that either favor some form of unified model or at least do not strongly contradict such models.

However, although it is certain that viewing angle has affected our classification of radio galaxies and quasars, precise and unambiguous tests of the hypothesis that all objects in these separate apparent classes belong to the same intrinsic population are difficult. The total sample of objects cannot be wholly homogeneous, and the objects must have a certain dispersion in physical properties such as opening angle; in addition, various insidious selection effects from the beamed properties of the objects may bias samples chosen to measure isotropic properties.

Recent successes at resolving quasars at $z>1$ have prompted us to undertake a project to image a complete sample of 15 quasars at $z \sim 1$ in order to address the issues of the relationship between radio galaxies and quasars and, possibly, of the alignment effect in high redshift radio sources. We seek to minimize the bias in our samples by selecting on radio-lobe properties alone. We have taken deep WFPC2 imaging of a small but complete subset of these quasars and the matched complete sample of radio galaxies in the hope that the higher resolution of HST will enable us to make our comparison of radio galaxy and quasar host properties less hindered by systematic effects than previous ground-based studies. We present here the results of the WFPC2 and groundbased imaging of this HST subsample; the remainder of the larger sample will be discussed elsewhere.

\section{Sample Selection}

The revised 3C (3CR: Bennett 1962) catalog has long provided the only large sample of radio sources selected at low frequency for which both optical identifications and redshifts are now essentially complete (Spinrad et al. 1985). For this reason, we have concentrated on $3 \mathrm{CR}$ objects to form our samples. Our larger complete sample of 3CR quasars is chosen to match the 3CR galaxy sample of Rigler et al. (1992) in extended 
radio properties and in redshift range. We provisionally included all 3CR quasars in the range $0.8<z<1.25$ with steep-spectrum fluxes above the survey limit of $9 \mathrm{Jy}$ at 178 $\mathrm{MHz}$. We define as "steep-spectrum" objects with spectral indices $\alpha$ (computed at 2700 $\mathrm{MHz}$; see note to Table 1) $>0.5$, where $S_{\nu} \propto \nu^{-\alpha}$. Our final sample was limited to those steep-spectrum quasars whose total flux is not brought above the survey limit by a flat spectrum compact core, in order to try to ensure that we are selecting on an isotropic property. The extended, steep-spectrum lobes of FR II radio galaxies are optically thin and probably mostly unbeamed. While Garrington et al. (1991) found that the spectral index is flatter on the jet-side, consistent with a beamed contribution to the lobe flux, Blundell \& Alexander (1994) have argued that this spectral index asymmetry may be explainable simply by projection and light-travel-time effects, in that the radio emission from the more distant lobe should have age-steepened more than the emission from the nearer lobe. In any case, the contribution is considered minor (Urry \& Padovani 1995), and it is probably not a significant source of bias in our radio-lobe-luminosity selected sample.

For our HST observations, we have defined a complete subset of the above $z \sim 13 \mathrm{C}$ sample. We include all $3 \mathrm{CR}$ objects that fulfill the radio flux, morphology and spectral index constraints of the quasar sample, as well as having a redshift within the range $0.87<z<1.05$ and a $\delta<60^{\circ}$. The lower declination limit of the revised 3C survey is $-5^{\circ}$; our sample could equally well have been drawn from the further-restricted sample of Laing, Riley, \& Longair (LRL; 1983), except for our inclusion of $3 \mathrm{C} 2$ and $3 \mathrm{C} 237$, which have declinations below the LRL declination limit of $10^{\circ}$.

The redshift range for our sample was chosen so that the WFPC2 filters F622W and F675W give passbands centered near rest-frame $3300 \AA$ with little or no contamination from emission lines. After deleting $3 \mathrm{C} 22$ because of high extinction $\left(A_{B}=1.09\right)$ along the line of sight, we are left with a sample of 5 radio galaxies and 5 quasars. These are listed in Table 1 along with some information about their optical and radio properties. The 10 sources all have double-lobed radio structure; eight have largest angular sizes (LAS; from lobe hotspot to hotspot) $\geq 7^{\prime \prime} ; 3 \mathrm{C} 2$ has LAS $\sim 5^{\prime \prime}$ and has sometimes been termed a "compact steep spectrum" (CSS) source (Saikia et al. 1987). 3C 237, with a LAS of $\sim 1$ ".3, is included in most samples of CSS objects (e.g., Fanti et al. 1990). 


\section{Observations and Reduction}

\subsection{HST WFPC2 Imaging}

Table 2 gives the log of HST observations. Total exposure times for each object in our sample were calculated to give roughly the same signal-to-noise level to a given proper surface brightness limit; actual total integration for our WFPC2 observations are given in Table 2. Our general technique was to take two, three, or four separate exposures of 900-1100 seconds at the same pointing to aid in removal of cosmic rays, but to dither the telescope in a square pattern 10.5 pixels on a side between subsequent sets of exposures to aid in removal of hot pixels and to improve our sampling of the PSF. The object was centered on WFC3, giving a scale of $0{ }^{\prime} 1$ pixel $^{-1}$ (undersampling the HST point-spread function). HST points accurately to $\sim 3$ mas $(0.03$ pixel $)$, and we found that using the intended pointings to shift and combine the dithered images gave better final image quality than re-assessing the offsets by centering on the undersampled stars.

We tried recalibrating the raw data with several different types of STScI-provided bias-dark combinations and found the best standard deviation in final combined frames from using the pipeline-supplied biases (averaged from 40 individual bias frames close in time to the observations) and a super-dark (an average of 100 darks). The recalibration routine generates bias-subtracted, dark-subtracted, and flattened files, as well as associated bad pixel masks (data quality or "DQF") files. We resampled the images by a factor of two to a size of $1600 \times 1600$, then aligned by shifting in integer (resampled) pixels. For combination and cosmic ray removal, we used primarily the STSDAS gcombine routine. We first rejected all pixels marked as bad or saturated in the DQF files generated by the recalibration routine. We then rejected pixels that were more than $3 \sigma$ from the median, using the rejection algorithm "rsigclip" to compute a median and sigma that are robust against unidentified outliers. We then compute each output pixel value from the average of the remaining unrejected pixels. From all summed frames in which we had unsaturated stars, we measured an average stellar full width at half maximum (FWHM) of $0{ }^{\prime} 14$ with a $1 \sigma$ variation of 0.02 .

We have derived the flux density in our images from the calibration information provided by STScI, i.e., from the PHOTLAM calibration keyword generated by SYNPHOT in the pipeline calibration. These calibrations are determined from knowledge of the instrumental sensitivities and filter bandpasses, supplemented by WFPC2 imaging of known standards. We normally will give our photometric results in terms of flux densities for the optical images in this paper, since we use non-standard filters. 


\subsection{Ground-based Optical Imaging}

The ground-based observations are summarized in Table 3. Our non-standard optical filters are designated by $\{$ central wavelength $\} /\{$ FWHM $\}$, where both quantities are given in Ångströms. The optical continuum images were taken at the University of Hawaii 2.2-m telescope with an anti-reflection-coated, thinned Tektronix $2048 \times 2048$ (Tek2048) CCD. While we have ground-based images of all of the quasars in our sample obtained with filters very similar to the WFPC2 filters, we used these images mainly for consistency checks on the HST calibrations; we will make no further use of them in this paper. For $3 \mathrm{C} 2$ we also obtained an image in a redder passband near Mould $I$ (our 8964/1063 filter).

We also observed 4 quasars and 2 of the radio galaxies in narrow-band [O II] interference filters. These filters are high-transmission $(\sim 90 \%)$, square-bandpass filters with widths $\sim 30 \AA$, centered at redshifted $\lambda 3727 \AA$ for each of the objects. The narrow-band images were taken at UH $2.2 \mathrm{~m}$ with the Tek2048 CCD and at the CFHT with SIS fast guiding and the Orbit1 (Orb2048) CCD; specifics of the seeing conditions (FWHM) and filter positions can be found in Table 3 .

We followed standard CCD data reduction procedures, with a few adjustments for some of the peculiarities of the detectors. For the continuum images, we made sky flats from the median average of the dithered, bias-subtracted raw images (while masking objects out of the median). We compared the results of using sky flats versus dome flats in flattening the raw frames; in general, the sky flats worked better. In a few cases, bright stars left residuals that we could not successfully mask out, and in this situation we adopted the dome flats. Each frame was corrected for atmospheric extinction using mean extinction coefficients. The narrow-band images were treated similarly, except that, because we needed long exposures to approach sky-noise-limited statistics in the background, we normally obtained only 3 dithered frames and could not construct good sky flats; we therefore used dome flats instead.

To produce our final CCD images, we median-averaged the separate frames after aligning each to an accuracy of $\sim 0.1$ pixel $\left(0^{\prime} 02\right)$, using the brightest unsaturated stars in each field. We then flux-calibrated using Kitt Peak spectrophotometric standard stars with data to $1 \mu \mathrm{m}$ (Massey et al. 1988; Massey \& Gronwall 1990). To determine the standard star flux in our non-standard continuum and narrowband filters, we approximated our filters as square with width equal to the FWHM of the filter profile, and integrated the stellar spectral flux within this region. 


\subsection{Infrared Imaging}

The near-infrared images were taken at the UH $2.2 \mathrm{~m}, \mathrm{CFH}, \mathrm{IRTF}$, and Keck telescopes. All objects were observed at Mauna Kea $K^{\prime}$ (Wainscoat \& Cowie 1992). This filter is centered at $2.1 \mu \mathrm{m}$ (shorter than standard $K$ ) in order to reduce thermal background. A few objects were observed at standard $H$ as well, which is centered at $1.65 \mu \mathrm{m}$. At the UH $2.2 \mathrm{~m}$, we used the NICMOS3 $256 \times 256(\mathrm{Nic} 256) \mathrm{HgCdTe}$ infrared array at $\mathrm{f} / 10$, with a pixel scale of $0.37 \mathrm{pixel}^{-1}$, and at $\mathrm{f} / 31$, with 0.12 pixel $^{-1}$. We also used the $1024 \times 1024$ QUIRC (Qrc1024) HgCdTe camera, with a scale of $0.18 \mathrm{pixel}^{-1}$ at $\mathrm{f} / 10$. At the CFHT, we used the UH NICMOS camera in March 1992, with a scale of $0^{\prime \prime} 3$ pixel $^{-1}$ and the CFHT Redeye Camera, (also a $256 \times 256$ NICMOS3 device), in November 1993 with a scale of 0.2 pixel $^{-1}$. The IRTF observations were made with NSFCam, a $256 \times 256$ InSb array. NSFCam has an adjustable pixel scale; we chose to use 0.15 pixel $^{-1}$ and 0.3 pixel $^{-1}$ on separate occasions. The Keck Near-IR Camera (NIRC) also uses a $256 \times 256$ InSb device. Seeing and photometric conditions were variable; best seeing was 0.5 FWHM and worst $\sim 1$. 3 . We give the specifics of each observation in Table 3 . We offset the telescope slightly between each exposure on a field to facilitate creation of sky flats and removal of bad pixels. Dome flats were made by subtracting observations of the incandescent-illuminated dome from observations of the dome with the lights off. Except at Keck, the standard readout procedure for these devices leaves little or no bias.

We outline our reduction procedure for the UH $2.2 \mathrm{~m}$ NICMOS3 observations, and afterwards indicate variations made for other devices. An iterative process was used to flatten the data and replace the bad pixels. First a bad pixel mask was created from the dark frames and dome flat field frames. The raw object frames were then normalized by their median sky value and combined to create a sky frame. (Every calculation of a median sky value excluded the masked-off regions associated with that frame). This sky frame was then scaled to each raw frame and subtracted. The subtracted frames were flattened with the dome flats. These rough flattened images were aligned to the nearest pixel using stars or the quasar itself and median-averaged to create a rough combined image. A mask was made from this combined frame of the positions and extents of the objects. The portion of this object mask that was associated with each individual frame was added to the bad pixel mask to create a combined mask for that frame. The process was then repeated; using the object mask to mask objects out of the sky frame, we made superior flattened frames, recalculated the centering and alignments, and made a better combined image. This combined image was then used to replace the bad pixels in each flattened frame with the median of good pixels from the rest of the frames. The bad-pixel-corrected flattened frames were then interpolated and resampled; since most of our infrared data are undersampled, the magnification factor was generally in the range 4-8. The centering was recalculated 
(bad pixels may badly skew centering), as were the fluxes of a number of photometric reference objects we had previously specified. Using these photometric fluxes to scale each frame to the median flux value, we made a final combined frame, using sub-pixel alignment with an accuracy of $\sim 0.2$ pixel ( $\sim 0^{\prime} .07$ for the UH NICMOS images). In cases where we had variable extinction from clouds, we scaled the individual frames to the maximum flux recorded. This process treats incorrectly any contribution from the dark current, which was scaled along with the sky, but the effect is negligible as long as the dark contribution is a small percentage of the total background. For the NICMOS chip, the uncertainty in the level of dark to subtract is greater than any resulting uncertainty this process may add.

The $1024 \times 1024$ QUIRC chip (at the UH $2.2 \mathrm{~m}$ ) has a much stabler dark pattern, and we generally subtracted a dark frame from the object exposures prior to creation of the sky flat. With NIRC at Keck, twilight sky flats were used instead of dome flats. A bias must be subtracted, which we obtained from dark frames taken at the beginning and end of each night. The scale is $0.15 \mathrm{pix}^{-1}$, resulting in a field of view of $38^{\prime \prime}$. This meant that we were not able to have a star on the frame in all cases. Otherwise reduction procedures followed were similar to that for the NICMOS at the UH $2.2 \mathrm{~m}$.

We calibrated our near-infrared data with standards from the UKIRT faint standard list (Casali \& Hawarden 1992) and from the Elias et al. (1982) list of moderately bright standards. We corrected for the color difference between $K$ and $K^{\prime}$ as prescribed by Wainscoat \& Cowie (1992); the resulting flux difference was $\sim 2-3 \%$, which is generally less than the error in our absolute calibration. A secondary check on our absolute calibrations can be made by comparing fluxes of stars from separate observations of the same field. Since we have Keck observations for all objects except for $3 \mathrm{C} 2$, all other observations are referenced to our Keck data. The average percentage difference in absolute calibration is $0.4 \%$, with a standard deviation of $5.1 \%$, and a maximum mismatch of $12 \%$; this standard deviation flux difference corresponds to a magnitude difference of 0.05 .

\section{Analysis}

\subsection{Point Spread Function Determination}

To determine the morphologies and magnitudes of the extended material underlying the quasar nuclei, we must remove the contribution of the nuclear component. We describe here some of the details of how we determine the point spread function (PSF) that we use to subtract off this nucleus. 


\subsubsection{The HST Point Spread Function}

For the HST data, we observed a PSF star in association with each of the quasar observations. We planned the exposures to try to match as closely as possible the observational procedure for the quasars themselves; e.g., we followed the same dithering procedure we discuss in $\S 3.1$ and calculated the PSF exposure times in order to saturate the PSF to the same extent that we saturated on the quasars themselves. In Table 4, we give the PSF star we observed in conjunction with each quasar, the filter in which each was taken, the integration times, and the greatest extent of saturation in any dimension for both the QSO and PSF. As can be seen, our predictions of QSO and PSF magnitudes were not quite perfect, and the saturation diameters range from 0 to 0 '25. We note here that these saturation regions are the intersection of whatever pixels were marked as A-to-D-converter-saturated by the DQF files; we mask out entirely these saturated pixels and replace those data with other data values from the stack of images if other pixels are not saturated. Therefore, because we dithered our exposures by half-pixel steps, and saturation may vary slightly from exposure to exposure, we may end up with a saturated diameter of 1 resampled pixel $\left(0^{\prime \prime} 05\right)$, which is equivalent to half an original resolution element of the WFC.

The PSF varies across the field, so we checked the observed QSO and PSF positions on the chip. This positioning was very stable: the variation in the distance from the mean column position for all PSF stars and QSOs was $\sigma_{\text {column }}=0$ ' 35 , and the maximum deviation was 0.7 , for the $3 \mathrm{C} 245 \mathrm{PSF}$ star. The row positioning varied a little more $\left(\sigma_{\text {row }}=0\right.$ "' 6$)$, but the maximum deviation from the mean position was $1^{\prime \prime} .2$ for the PSF star of $3 \mathrm{C} 336$. As we have more than one measurement of the PSF in each filter, and our mean positions on the frame do not vary much, we can now consider averaging the PSFs in each filter to increase our signal-to-noise. In order to assess how much the PSFs differ from each other, we determined their relative centers and scalings in annuli outside of their respective saturated regions and subtracted them. We use the same centering and subtraction techniques (discussed in §2) that we used for subtracting the PSFs from the quasars, though these cases are of course not complicated by the existence of extensions. The PSFs in the same filter subtract well from each other with few systematic residuals. The inner $0^{\prime \prime} 35-0{ }^{\prime \prime} 45$ radius is very noisy, and we find in the subsequent quasar-minus-PSF subtractions that we are unable to recover much information from this region, even when it is not saturated. We note that the diffraction spikes (especially in the region close to the center of the PSF) may leave some residuals. We show some examples of these PSF-minus-PSF subtractions in Fig. $1 A-C$. We then average the 2 F622W PSFs, associated with quasars $3 \mathrm{C} 196$ and $3 \mathrm{C} 336$, and the $3 \mathrm{~F} 675 \mathrm{~W}$ PSFs, associated with quasars $3 \mathrm{C} 2,3 \mathrm{C} 212$, 3C 245 , using the normalizations and centers determined from the subtraction technique. We mask out the 
saturated regions in making the combined PSFs, and replace saturated values with values from unsaturated stars in the average if available.

We display in Fig. $1 D$ the combined PSF for the F622W filter (the average of the 3C 196 and 3C 336 PSF stars). We have also checked the residual of the difference between the two average PSFs. As expected, we find that there are significant differences between the two filters; we did not, therefore, average all of the PSFs together.

\subsubsection{The Infrared Point Spread Function}

The ground-based PSFs are, of course, very dependent on the details of the atmospheric conditions throughout the observations, and they must be determined from stars taken as nearly simultaneously as possible. For both the Keck and CFHT images that we present here, the observational field is small enough that we must usually take exposures of a star (of comparable brightness to the quasar) interleaved with the object observations in order to determine the PSF. The one exception in the quasar sample is $3 \mathrm{C} 212$, which has a star of sufficient brightness within the NIRC field. We also obtained PSF stars for the Keck observations of the radio galaxies. (For the radio galaxies, the star need not be so bright; there were therefore unsaturated stars of sufficient brightness on each radio galaxy field except for 3C 280 and 3 C 217).

When we must interleave observations of a PSF star with the actual object integrations, we want to sample as well as possible the seeing conditions and any PSF field variations. We therefore bracketed the object observations with observations of the PSF, and alternated between the PSF star and object as often as efficiency considerations allowed (generally within 15 minutes). We used the same dithering pattern in the two sets of integrations to make sure that any PSF variations in the field or systematic effects caused by our dithering and centering technique are similar in the two cases; we also used the same effective individual integration times where possible. Despite these precautions, natural variations in the ground-based seeing conditions result in some systematic PSF residuals; these are generally obvious and confined to the inner region of the seeing disk.

\subsection{Point-Spread-Function Subtraction}

To determine the morphologies and magnitudes of the extended material underlying the quasar nuclei, we must remove the contribution of the nuclear component. We will discuss some of the uncertainties in this process, and some differences between the ground-based 
and HST data. (All of the PSF subtraction was done in the original combined images, before subsequent rotations and transformations that would smooth the images and affect their pixel-to-pixel noise characteristics).

Some information is not recoverable from the PSF subtraction process; for example, a compact peaked host galaxy might be indistinguishable from extra flux in the PSF and would be subtracted. We can, however, make reasonable assumptions about the probable behavior of the host galaxy and use these to estimate the total magnitude of the nebulosity. The simplest technique is to fit the PSF to the quasar nuclear component in a specified inner region, subtracting the quasar flux in this region to zero.

Subtraction to zero should give a lower limit to the magnitude of the extended material if any exists but will oversubtract if there is any extended flux in the inner region. Some simulations of ground-based observations of low-redshift quasars, where the host galaxies were assumed to be normal spirals and ellipticals, found that subtraction to zero decrease derived magnitudes by $\sim 0.5$ mag or more (Abraham et al. 1992). A more realistic criterion would be to require that the host galaxy increase monotonically or at least remain constant from the outer regions that are mostly unaffected by the PSF subtraction into the interior dominated by the PSF. Even this monotonicity criterion is likely to underestimate the total host galaxy flux, if the galaxy peaks at all in the center, as would a normal spiral or elliptical. We calculate two subtraction limits for our quasars, both a subtraction-to-zero lower limit, and a monotonic-across-inner-region best estimate. In those objects in which we find little or lumpy extended flux, these two cases end up essentially the same.

In the interests of objectivity, we have tried to automate the subtraction process, though visual inspection of the subtraction residuals remains a useful cross-check on the process. We have developed IRAF scripts that allow us to center and scale the PSF to the quasar, display the residuals in a defined annulus, and calculate the reduced $\chi^{2}$ of the PSF fit to the quasar. We first estimate the proper centering and scaling by calculating the fluxes and the $x$ and $y$ first moments of both the PSF and the quasar in an inner defined annulus, correcting for partial pixels. The annulus used is one of the most subjective parts of this process: it is chosen to exclude any saturated interior region, to be small enough to minimize the host galaxy contribution, yet large enough to provide decent statistics. For the HST data, the inner radius we use varies from $0^{\prime \prime} 0-0^{\prime \prime} 35$, and the outer from $0.2-0.45$. Our HST data are only minimally saturated (several pixels or 0.25 at the most; see Table 4).

We then optimize the centering by calculating the the $\chi^{2}$ values of the difference in the defined annulus over a grid of $x$ and $y$ shifts; we reject pixels from the $\chi^{2}$ sum that deviate by $3 \sigma$ from the mean difference value to reduce the effect of intrinsic PSF/QSO 
shape differences. We fit a quadratic to each of the $\chi^{2}$ vs. $\delta x, \delta y$ plots, and we take the minima as the optimum $\delta x, \delta y$. Though this procedure incorrectly treats the $\delta x, \delta y$ and the QSO-to-PSF scaling as independent, the centering does not change significantly with any reasonable scaling. In addition, the centering is well-determined, while the scaling is a much more arbitrary and subjective quantity. When we center by hand, by inspecting the residuals, the best center is determined to about $0^{\prime \prime} 01$, and it matches the results of automatic centering to within this tolerance.

To obtain the best scaling, we use a minimization technique similar to that we use for the centering: we vary the scaling around the initial value, using the already determined best center, then fit a quadratic. In Fig. 2 we show the determination of the $\chi^{2}$ minimum scaling for the HST image of $3 \mathrm{C} 2$. The minimum $\chi^{2}$ corresponds to the "best fit" between the QSO and the PSF two-dimensional distributions; as discussed above it is probably generally an oversubtraction of any extended flux, but provides a lower limit to the host galaxy flux. Further evidence of this is the fact that the minimum $\chi^{2}$ is usually reached at different scalings depending on what annulus is chosen; using annuli including data at a greater radial distance from the QSO center causes the QSO:PSF scaling ratio to increase, as may be expected if the QSO is contributing more extended material than the PSF star at these radii. In part, we have adopted this approach of estimating the $\chi^{2}$ minimum of the fit in order to provide a good comparison to the Bahcall et al. $(1994,1995 a, b, 1996,1997)$ results, in which the $\chi^{2}$ fit is minimized with respect to 3 variables: the $x$ and $y$ shifts between the quasar and PSF, and the QSO:PSF scaling. These subtractions correspond, therefore, to "subtraction-to-zero" lower limits; similar conservative limits are used by Heckman et al. (1992), and Lehnert et al. (1992). We also wish to automate an objective version of the "monotonicity" constraint; this is similar to the approach used in a variety of studies of low- $z$ quasars, such as the HST study by Disney et al. (1995) and the groundbased studies of McLeod \& Rieke $(1994 a, b)$. To establish the monotonicity of the profile difference requires averaging the difference profile in annuli with some width; generally this will be equivalent to requiring our interior annulus to have a mean value equal to the annulus immediately exterior to it. We therefore define an annulus exterior to our inner annulus, and record the mean and median of the values in both the inner and outer annuli (after $3 \sigma$ rejection, to minimize the effects of PSF residuals in skewing the mean and median). We once again desire to sample as narrow a width as possible of the profile that will still give signal-to-noise adequate to prevent the scaling being dominated by statistical noise. These outer annuli had widths from $0{ }^{\prime} 1-0,2$ for the HST images. Any montonicity constraint applied requires some such assumption; the wider the annular width used, the more host galaxy is subtracted. We check the average values in the profile difference in annular steps of this width for monotonic behavior, but it is the inner two areas that set the scaling. In Fig. 
2 we show how we automate this constraint; we plot the decreasing average values in the two inner annuli and take as the scaling the point at which lines fit to these two basically linear functions intersect. This gives us a better estimate of the proper subtraction, and if there is no extension, the $\chi^{2}$ minimum scaling and the monotonic limit scaling are the same. In addition, much of the residual structure is lumpy and irregular, and when we take a radial radially, the mean value in the annulus is much less than the intrinsic surface brightness of the clumps.

We subtracted all of our ground-based quasar images in an identical fashion, forming both the $\chi^{2}$ minimum limit and monotonic limit. As the ground-based observations were never saturated, we were able to use the very central portion of the profiles, and varied the annular widths according to the seeing. To give a specific example of radii chosen, for the $\mathrm{CFH} K^{\prime}$ observations of $3 \mathrm{C} 2$, we used the circular region within 0.4 to calculate the $\chi^{2}$ minimum scaling and an annulus with width 0.4 exterior to that to calculate the scaling which satisfies the monotonicity constraint. We have checked our subtraction techniques by subtracting PSF stars from each other, as mentioned in $\S 4$. We have made other consistency checks on our PSF subtraction results: for the HST data, we have subtracted each quasar both with the average PSF for its filter and with the PSF observed close in time, and found no systematic differences. Detailed discussion of the individual objects will be presented in $\S 5$.

\subsection{Magnitudes and Colors}

In this section, we outline our general approach for determining magnitudes and colors. Details of our magnitude and moment analysis are given in Appendix A. Magnitudes and colors of discrete components in the fields of our sample are discussed under the individual object descriptions in $\S 5$ and in $\S 6.3$, and global properties of the extended material are given in $\S 6.1$ and $\S 6.2$.

\subsubsection{Total and Isophotal Magnitudes}

For the WFPC2 imaging, we planned our exposure times to achieve similar detection limits in all of our 10 images, after normalization for redshift and reddening. In Table 2, we give the $1 \sigma$ detection limit we actually achieved in our WFPC2 images for each of our 10 objects, after normalization to $z=1$ and $\mathrm{E}(B-V)=0$. To correct for Galactic extinction we obtained $\mathrm{E}(B-V)$ values for our sources from Burstein \& Heiles (1984) and converted 
these to Galactic extinctions at the appropriate HST filters (F622W and F675W) using the values given in Table 12A of Holtzmann et al. (1995). For each object, we aligned all our ground-based images to the HST scale and reference frame, using the IRAF tasks geomap and register. This reference frame is in general rotated from standard astronomical position angle.

Since our primary goal is to make an objective comparison between the radio galaxy and quasar sample properties, ideally we would compare magnitudes and morphologies at identical isophotal flux limits (after normalization to a common reference redshift). In practice this procedure is complicated by the very irregular nature of the extended material we have resolved around the quasars; unresolved or linear features do not lend themselves so well to isophotal analysis as smoothly varying galaxies. For this reason, we also will calculate simple aperture and annular magnitudes.

After subtraction of the PSF, we normalize the images to $z=1$ and make a series of masks from the normalized images corresponding to isophotal levels of interest. We investigate magnitudes and moments in apertures with no flux limit, and in apertures defined by standardized surface brightness cutoff levels. When we discuss the properties of a PSF-subtracted quasar, we generally refer to the results from the monotonic-subtractionlimit; however, we use the $\chi^{2}$ minimum fit as useful check, both as a means of error estimation and as an indication of how dependent on our PSF subtraction method our results may be.

We have also PSF-subtracted the HST radio galaxy images with the best combined PSF from the appropriate filter, and verified that outside of the excluded central region, the wings of the PSF do not significantly contribute to the radio galaxy annular fluxes. For the near-infrared radio galaxy images, the extended flux is mostly quite dominant, and we estimate an upper limit to the unresolved contribution by subtracting until the the residual galaxy profile obviously is no longer monotonic. In the ground-based images, the amount of flux removed is very seeing dependent, and this upper limit is probably an overestimate of the unresolved contribution, nonetheless the wings of the PSF contribute little to the annular fluxes outside of the seeing radius.

\subsubsection{Colors and Spectral Energy Distributions}

Since we are dealing here with images having quite different resolutions, we take a two-step approach to determining the magnitudes and colors of various components in the images. Inspection of the original HST image can be used to identify components that 
occur close to the quasar nucleus and may not be easily distinguishable at the resolution of the ground-based data. We then smooth the HST image to match the resolution of the ground-based image in question, so that an aperture will encompass a similar portion of the component's flux. We will then use the same apertures (simple or tailored) in each bandpass. There is generally a straightforward correspondence between the HST data and the ground-based data, but sometimes components that may be resolved from others in the HST images cannot be identified clearly in the ground-based images, which differ not only in resolution but in bandpass. Thus we cannot get a $K^{\prime}$ magnitude for every component for which we have HST fluxes; in cases for which the residuals from the subtraction process obscure the component at ground-based seeing, we cannot even obtain a useful upper limit. The core fluxes and limits are derived as a result of the PSF-subtraction process, and represent the monotonic limit.

\section{Discussion of Individual Fields}

In this section we present and discuss the HST optical and ground-based $K^{\prime}$ imaging of our 10 sources. We will discuss their morphologies and give photometry for components of interest, but we will reserve the discussion of the quantification of the alignment effect and the properties of the sample as a whole for later. In Figs. 3-12, we show the HST image and the $K^{\prime}$ image at the same scale in the upper left and upper right panels, respectively. In the lower left panel we display a magnified version of the HST image, sometimes slightly Gaussian smoothed to bring up lower surface-brightness features. In the lower right panel, we display the continuum-subtracted [O II] image, if we have one for that object; if not, we generally display another version of the HST image, scaled or smoothed differently, or with radio map contours overlaid. In the top left panel, we give the positions of the radio lobes as white crosses (or show the direction to them as white arrows, if they lie outside the frame). Throughout the images, insets will display different intensity scalings or a non-PSF-subtracted version of a quasar. In all cases, $\mathrm{N}$ is at the top, and $\mathrm{E}$ is to the left. In Tables 5 and 6 , we give photometry for the components labelled in the figures.

\section{1. $3 \mathrm{C} 2$}

\subsubsection{Morphology}

$3 \mathrm{C} 2$ has (relative to its nucleus) the most obvious extension of the quasars in our sample; it shows clear signs of "fuzziness" even in groundbased unsubtracted images, 
especially in the $K^{\prime}$ image. The flux of the extension itself is roughly comparable to those of the other quasars, but it has the faintest nucleus of the 5 quasars we observed; the ratio of the optical flux of extension to that of the quasar nucleus is $\sim 0.15$ for $3 \mathrm{C} 2$, and from 0.01-0.03 for the other quasars. In Fig. $3 A$ and $B$, we display the HST and CFHT $K^{\prime}$ images; the PSF-subtracted images show the monotonic limit. We label as $a, b$, and $c$ the components within a few arcseconds that can be identified above the general nebulosity. Object $a$ has a nearly stellar peak at a distance of 0.83 and a position angle (PA) of $11^{\circ}$. We find it to be precisely coincident with the northern radio lobe of $3 \mathrm{C} 2$.

The radio structure of $3 \mathrm{C} 2$ consists of this northern lobe and a southern lobe at a PA of $-160^{\circ}$ and a mean distance of $4^{\prime \prime} 5$ (Saikia et al. 1987). From Saikia et al.'s 2-cm map of the northern component, which has a similar resolution to our HST images $\left(0^{\prime}, 16 \times 00^{\prime} 10\right)$ we can see that even the details of the morphology of the northern radio component and the optical component a agree. The WFPC2 image shows a similar extension directly north, then a turn to the east to a bright peak. When we align the optical quasar and the radio core, the bright optical peak and bright radio knot align to within 0 . 02 . The optical image has the same fainter north-eastern extension that is seen in the radio map. (We display in an inset in Fig. $3 C$ two contours associated with the northern lobe from the $2 \mathrm{~cm}$ radio map of Saikia et al. [1987]).

Though object $a$ has a separate, unresolved peak in the WFPC2 data and therefore might have been considered a companion object on the basis of the optical data alone, the close optical-radio lobe coincidence and the "bridge" of connecting nebulosity imply a close association with the quasar. Object $c$, on the other hand, seems morphologically discrete (though it is also enveloped in the general nebulosity surrounding the nucleus). Object $b$ is diffuse and seems to connect into the noise-dominated inner region, and it shows no correlation with the radio structure. Of the three objects we have labelled, it is the only one that might be a feature of the host galaxy itself.

The sky level for the 3C 2 HST observations was lower than expected; we reached a greater depth for $3 \mathrm{C} 2$ and for $3 \mathrm{C} 217$ than for any other fields. The extended nebulosity we observe in $3 \mathrm{C} 2$ is mostly at a lower surface brightness than our lowest standardized contour level; it is possible therefore that a similar nebulosity might exist and have escaped detection in the objects in our sample that have a lower signal-to-noise background.

Our CFHT $K$-band data shows the three major components visible in the HST image as well as some extended nebulosity; our IRTF $H$ image shows similar structure but is not deep enough to show the general extended nebulosity. 


\subsubsection{C2 Component Spectral Energy Distributions}

For 3C 2, we have two extra passbands: $H$, and our $8964 / 1063$ filter, which is centered slightly redder than the $I$ passband. $3 \mathrm{C} 2$ is the only object in our sample for which we have 4 passbands spanning the rest-frame range from $3300 \AA$ to $1 \mu \mathrm{m}$. We will discuss here the spectral energy distributions of various components: the lobe structure labelled $a$ in Fig. $3 A$, the faint extension $b$, the nearby companion $c$, and the underlying nebulosity (seen best in the highest-contrast HST image panel, Fig. $3 D$ ). This faint extended structure appears relatively symmetric and has an angular extent of about $3^{\prime \prime}$ radius, as measured at the $2 \sigma$ sky level in the $K^{\prime}$ image, where it is brightest relative to the other components. In addition, we derive the SED of the quasar nucleus alone, with the contributions from the above components subtracted.

For the SED of the nebulosity, we adopt an annulus with inner radius 1".75 and outer radius $3^{\prime \prime}$. The fluxes within this radius, given in Table 6 , certainly underestimate the total fluxes of the nebulosity in each passband, but they should give the best color estimates for the extension. The signal-to-noise in the $H$ and $I$ band images is insufficient to make spatial maps of the color distribution.

For the discrete components we use a similar procedure. Components $a$ and $c$ are quite compact, and we measure their fluxes in apertures with radii equal to the FWHM of the degraded seeing, excluding, of course, the central 0.5 radius around the nucleus that is dominated by PSF residuals. We subtract off the background extension in the HST image before smoothing, then Gaussian smooth to the poorer resolution. This process may lead to systematic errors, so we estimate the background contribution in several different ways and use the dispersion as an estimate of our errors. The images with the poorest resolution have the greatest systematic uncertainty from the background subtraction process. We present the result of this photometric analysis, with errors, for all the components in Table 6 , and give there the exact apertures used for each component.

\subsubsection{Discussion}

Because of the detailed close correspondence between the optical and radio morphologies of the northern component $a$, it appears that the physical mechanism producing the optical radiation is directly related to the radio emission. To address the nature of this relation, and to investigate the likely origin of the other components we have resolved, we plot the flux values for each component values versus the rest wavelength (assuming that all objects are at the narrow-line redshift of the quasar) in Fig. $13 A$ and $B$. The fluxes are 
normalized to the flux value at $\lambda_{0}=3300 \AA$ (from the F675W HST image). We show both the subtracted and unsubtracted fluxes since this background subtraction process could be prone to large systematic errors. Comparison of the two plots shows that, although the details of each SED value change, each component's SED shows the same qualitative behavior with or without the background subtracted.

Though broad-band colors alone are a notoriously inaccurate way to derive information about the nature of any single object, we may still gain some indication of the probable origin of the radiation we observe and of the differences between the components. We see that the nebulosity and component $c$ are both redder than the QSO. This result is consistent with domination by a stellar population rather than by scattered quasar light. Lehnert et al. (1992) found a similar result for the extensions in $z \sim 2$ quasars. In panel $B$, we plot models representative of several stellar systems. None of these simple stellar models is a perfect fit, but component $c$ has a $4000 \AA$ break that is fit very well by the Bruzual \& Charlot (1993) models. This is evidence that it is at the redshift of the quasar and that it is stellar in origin. Reddening by dust, either internal or from the surrounding nebulosity, could explain the slightly redder colors in $c$ than in the 4 Gyr stellar models. The colors of the nebulosity are a poorer match for these basic stellar models; this could result from a significant contribution to the extended emission from a younger, bluer stellar population or perhaps from scattered quasar light. Component $b$ is of much lower flux, and very close to the nucleus; it was thus difficult to estimate its flux values, particularly for the two lower signal-to-noise images. Nonetheless, the colors are consistently bluer than either the nebulosity or $c$. Though we have suggested that it may be a feature of the host galaxy, its colors are consistent with a scattered light origin as well as with a young stellar population.

We also plot a short-dashed line in panel $B$; it is a power-law fit to component $a$ with $\alpha=1.36$, where $S_{\nu} \propto \nu^{-\alpha}$. (We weighted the fit with the errors on each point; for this reason, the $\lambda_{0}=0.44 \mu \mathrm{m}$ contributes little to the fit). This optical $\alpha$ is similar to that seen in optical synchrotron counterparts of radio hot-spots (Meisenheimer et al. 1989). In Fig. 14 , we show our photometry of the northern lobe (component $a$ ) with the radio photometry of Saikia et al. (1987) for the same component (crosses). We plot the power-law fit to our data (with $\alpha=1.36$ ) with a solid line; a linear fit to the Saikia et al. points gives $\alpha=0.83$, and is plotted as a dashed line. As we shall discuss more fully in $\S 8.3$, this break in the spectral index is more likely to point to optical synchrotron radiation than to competing explanations, such as inverse Compton scattering of microwave background photons. 


\section{2. $\quad 3 C 175.1$}

\subsubsection{Image Editing}

In the 3C 175.1 field, there are several bright stars near the galaxy, and these caused various artifacts that had to be removed manually prior to further analysis. In the HST image shown in Fig. 4, a diffraction spike from a bright star to the south has been subtracted out. Diffraction spikes are among the features in the HST PSF that vary most with position in the field, and we were more successful in subtracting an azimuthally averaged version of the star from itself rather than subtracting the observed PSF, which is accurate only for the center of the field. This process should have only added slightly to the noise, and there is no morphological feature in the radio galaxy that could be due to unsubtracted flux from the diffraction spike. In Keck NIRC images, bright stars bleed along rows. A bright star in the $3 \mathrm{C} 175.1$ field has bled through the radio galaxy; we subtract the extra flux by fitting a low-order polynomial along the row prior to rotating the images.

\subsubsection{Morphology}

Our HST image of this radio galaxy has an elongated component, in two parts, but it also has a strong nuclear source. The $K^{\prime}$ band data (both from Keck and CFHT) show a round component that coincides with the HST nuclear component, with more elongation at the lower isophotal levels. In the Keck image, the radial profile between 0.65 and 1".75 is consistent with a de Vaucouleurs $r^{\frac{1}{4}}$-law with an effective radius $r_{e}=1^{\prime \prime} 1 \approx 7 \mathrm{kpc}$. In addition to this symmetric profile, we barely detect a discrete component at the position of $a$, to the southwest. We show the HST and Keck images in Fig. $4 A$ and $B$ respectively. In panel $D$, we show the HST image with the contours of our $3.6 \mathrm{~cm}$ VLA map superimposed; we make a firm detection of an unresolved core and align this with the unresolved HST peak and near-infrared center. This $3.6 \mathrm{~cm}$ core does not correspond in position to the emission found at $6 \mathrm{~cm}$ by Neff, Roberts \& Hutchings (1995) that they identify as a possible core; we accordingly find that the source is less bent than they suggest. Ignoring the core, the PA between the two radio lobes is $70^{\circ}$; with the $3.6 \mathrm{~cm}$ core detection, the PA to the NE lobe is $78^{\circ}$ and the PA to the SW lobe is $-122^{\circ}$. The bending angle of the axis (as defined in Barthel \& Miley 1988) is $20^{\circ}$. The HST image shows no obvious alignment of the radio galaxy components and the positions of radio lobes. Nonetheless, some of the components of the optical image are interesting. The extension to the NE of the optical core is aligned approximately at the PA of the SW lobe; the linear feature $a$ is connected to the core by a ridge of emission which leads into the jet-contact point on the SW lobe. However, 
there is no detected radio emission underlying these features; most plausibly this optical morphology is the result of quasar light within a large opening angle being scattered off of shreds of material. Our moment analysis of the radio galaxy at $K^{\prime \prime}$ gives a PA that varies from $70^{\circ}$ to $90^{\circ}$ with isophotal cut-off level; the axial ratio over the same range is $0.93-0.98$. The result of Dunlop \& Peacock (1993) that the the $K$ band light is elongated with an aspect ratio of 1.48 , aligned at a $\mathrm{PA}$ of $70^{\circ}$ (well-aligned with previous assumptions of the radio PA) is clearly influenced by seeing conditions, causing them to include the seemingly discrete red companion objects to the NE. They conclude that possibly 3C 175.1 is more aligned in the IR than in the optical. This situation illustrates the difficulty of deciding what is appropriate to include in analyses of the alignment effect. However, whether or not these objects are actually associated with the radio galaxy, our WFPC2 image and VLA detection of the radio core indicate that there is no longer reason to conclude that the infrared is better aligned than the optical.

\section{3. $3 \mathrm{C} 196$}

\subsubsection{Morphology}

We display the PSF-subtracted HST and Keck images of this $z=0.87$ quasar in Fig. $5 A$ and $B$. This quasar has an obvious barred spiral galaxy to the southeast, also seen in the WFPC2 image of Cohen et al. (1996) and the PC image of Le Brun et al. (1997). This galaxy is probably the source of both the prominent $21-\mathrm{cm}$ absorption line system and the metal line absorption systems at $z=0.437$ in the quasar spectrum (Cohen at al. 1996; Le Brun et al. 1997; Boissé \& Boulade 1990, hereafter BB). As this is almost certainly a foreground object, we will exclude it when analyzing the other extensions we see around the quasar. This procedure is not so much of a problem with the excellent resolution of the WFPC2 data but is difficult in the analysis of the ground-based data.

3C 196 has a LAS (lobe hotspot to hotspot) of 5.'6 (Reid et al. 1995) but shows a fairly wide, extended lobe structure. The optical extension that we see to the north proceeds almost directly north for several arcseconds, then extends to the east at the lowest detectable level on our image. The lowest level extension has a slight knot of emission that is coincident with the north-eastern radio lobe (best seen in panel $C$ ). At higher isophotal levels, as seen in panel $A$ or the inset in panel $C$, component $a$ appears semi-discrete and is elongated in the direction of the northern radio lobe. In addition, as noted by $\mathrm{BB}$, there is a non-stellar object $(d) 6.9$ to the northeast that lines up approximately with the radio lobe direction and is elongated perpendicular to the radio axis. We see a similar object to the other side of the radio source, at a PA of $-172^{\circ}$, elongated basically parallel to $d$. We 
will call this second companion $e$; although it is out of the field included in panel $A$, it is quite similar in appearance to $d$.

Our [O II] image (in panel $D$ ) shows a bright, asymmetric emission-line region whose morphology (inset in panel $D$ ) corresponds fairly well to the optical structure. This correspondence tends to confirm that the peak of the $z=0.87$ line emission matches the location of $a$ and provides secondary confirmation that $a$ is at the redshift of the quasar, rather than the $z=0.437$ absorption line system. At the lower isophotes, the morphology is curved symmetrically to the east both north and south of the quasar.

\subsubsection{Discussion}

3C 196 represents one of the best examples in our sample of quasars of aligned structure in that is unlikely due to optical synchrotron emission. Components $a$ and $b$ are probably both associated with the quasar. They are fairly blue and are present in both the continuum and [O II] emission. This type of alignment is similar to that seen in high- $z$ radio galaxies. Possible contributors for the material seen here are thermal continuum and scattering of the quasar light off of ambient material.

BB bring up the possibility that 3C 196 could be lensed by the galaxy $c$. Though the [O II] morphology and the continuum morphology are distorted and arced, there is no report of a multiply imaged radio core. BB suggest that a secondary image of the quasar may lie within the image of the spiral; this suggestion is based on the elongation of the companion galaxy and a difference in morphology between their $B$ and $R$ band images. The elongation they note is probably just the bar of the spiral that is obvious in our higher resolution HST image; however, BB have color information and claim evidence for an extra blue component to the east of the center of the companion galaxy. We see a faint component in the bar of the spiral that might plausibly be associated with this nucleus but could equally well be intrinsic to the spiral galaxy. An inset in the NW corner of panel $A$ gives the unsubtracted quasar image at a lower contrast level, showing the bar of the spiral galaxy and the faint peak. This peak is most likely simply a knot or nucleus in the bar of the spiral galaxy.

Could there be lensing of the radio source itself? Lensing on this scale would probably require a large mass associated with a many- $L_{*}$ galaxy or a foreground group or cluster, but the spiral galaxy appears to be $\sim L_{*}$ if at $z=0.437$ (from our data and that of Cohen et al. 1996). We estimate using an isothermal sphere model with the AIPS task glens that the geometrical distortion induced in the lobes from lensing by an $L_{*}$ spiral is minor, and the resultant magnification factors are at most a few tens of percent. This makes it unlikely 
that $d$ is a gravitational arc, as also consisidered by BB. On our images, both $d$ and $e$ are elongated, but we see no strong morphological evidence for gravitational distortion.

$\mathrm{BB}$ find companion $d$ to be very blue in the optical and favor the interpretation that it is associated with the radio source (through jet-induced star formation), though it lies beyond the radio lobe. This suggestion is particularly interesting, given that we have an example of a somewhat similar situation in $3 \mathrm{C} 212(\S 5.4)$ : there we have an object (component $f$ in Fig. $6 A$ ) that falls directly on the radio axis and is morphologically similar (in the optical) to the radio lobe, yet lies $3^{\prime \prime}$ beyond it. However, the 3C 196 companions $d$ and $e$ have similar and very blue optical-to-infrared colors; the optical-to- $K^{\prime}$ flux ratios are 10.9 and 8.5, respectively, while the QSO itself has a flux ratio of only 4. As these objects appear very similar morphologically and in color, it is tempting to try to ascribe them to a similar origin. In this case, though the southern object is off of the radio axis by $20^{\circ}$, this is well within a typical quasar opening angle (Barthel 1989; Saikia \& Kulkarni 1995), and the blue objects may result from quasar light being scattered in dusty galaxies (this possibility was also considered by BB). In qualitative support of this explanation, the peak surface brightness in the more distant companion is $\sim 0.25$ the surface brightness of the nearer. In $3 \mathrm{C} 280$, there may also be morphological evidence of quasar radiation impinging on and scattering from an existing structure. However, in $3 \mathrm{C} 196$, we see no [O II] emission from these objects at the redshift of the quasar, making this explanation much less likely.

\section{4. $3 \mathrm{C} 212$}

\subsubsection{Morphology}

Even a casual inspection of the ground-based images of $3 \mathrm{C} 212$ shows an unusual clustering of objects around the quasar. With the high resolution of the HST image we see that several of these objects have morphologies and locations that make it likely that they are directly associated with the quasar and its radio jet. Here we have a clearcut example of a quasar with optical and near-infrared continuum structures that are aligned with the radio axis; although we see some degree of alignment in each of our 5 quasars, $3 \mathrm{C} 212$ is certainly our most spectacular example. In Fig. $6 A$ and $B$ we show the HST and Keck $K^{\prime}$ images; in panel $C$ we show the HST image magnified $2 \times$, and in panel $D$ the same image Gaussian smoothed to bring up low surface brightness features. The crosses in Fig. $6 \mathrm{~A}$ indicate the positions of the radio lobes in the $6 \mathrm{~cm}$ MERLIN map of Akujor et al. (1991).

Close to the nucleus, we see a general nebulous extension, but the most striking features are the three discrete blobs $a, b$, and $c$, which extend almost directly toward the 
NW radio lobe. They are compact but resolved ( $b$, the brightest, has a FWHM $\sim 0$ '25) and fairly blue. Though they are not resolved in the Keck image, there is a narrow extension in the $K^{\prime}$ band structure at the positions of $b$ and $c$.

At larger distances from the nucleus, features $f$ and $g$ are also closely aligned with the radio axis, but they lie just beyond the radio lobes. Object $g$ is fairly regular and compact, though with some wispy extensions we will discuss in more detail later. Object $f$, however, shows a structure that is extremely unusual (even in the context of the range of morphologies exhibited by objects in other deep WFPC2 fields) and reinforces the impression that it may be associated with the radio source. In fact, its optical image looks very much like a VLA image of a radio lobe: it even has a "hot spot," barely resolved $(F W H M=0 ! 17)$, near its tip and closely aligned with the three inner knots $a, b$, and $c$. The more diffuse component of $f$ has a tail-like shape, with a sharp outer boundary but a gradual fading away in the direction of the quasar. When we ratio the images of the component $f$ (after smoothing them to the same resolution), we find that $f$ exhibits a steep color gradient. This effect can be seen qualitatively by comparing the optical and $K^{\prime}$ images: the $K^{\prime} f$ component peaks $\sim 1^{\prime \prime} 8$ south of the optical point source. The $K^{\prime}$-to-optical flux ratio is $0.18 \pm 0.04$ at the optical peak and $0.98 \pm 0.09$ at the $K^{\prime}$ peak. Object $g$ is the reddest component, with a $K^{\prime \prime}$-to-optical flux ratio of 1.9 ; the colors of other components are fairly blue.

Thus, 3C 212 shows evidence for optical/IR components aligned with the radio structure both within and beyond the radio lobes. In view of the importance this object may have for our understanding of the nature of powerful FR II radio sources and of at least one version of the alignment effect, we have obtained deep multifrequency VLA maps and longslit spectroscopy with the Keck Low-Resolution Imaging Spectrometer (LRIS) for this field. Details will be given elsewhere (Stockton \& Ridgway 1997), but we summarize the most important conclusions from the present stage of the work here.

In Fig. 15 we show contours from the $3.6 \mathrm{~cm}$ VLA map overlain on the HST image; the radio and optical frames were registered with the quasar nucleus position. The bright optical objects $a, b$, and $c$ are seen to coincide precisely with knots in the radio jet. The close morphological tracking of these features implies an emission mechanism that links the radio and optical radiation directly, such as synchrotron radiation. In spite of the higher dynamic range of our new maps, there is still no evidence for radio emission beyond the lobes seen by Akujor et al. (1991). The $3.6 \mathrm{~cm}$ map shows a fairly broad, normal hotspot/lobe to the SE side of the quasar. The NW side has a straight jet; the NW lobe resolves into a chain of peaks, continuous on one side with the jet, which look like hot spots produced by the radio jet precessing over small angles. Interestingly, the morphology of this NW radio lobe appears to mirror closely that of the optical component $f$ lying $3^{\prime \prime}$ 
exterior to it. The morphological correspondence between the SE radio lobe and the optical component $g$ lying just beyond it is less striking, but the curved wisp extending $\mathrm{N}$ from the outer edge of $g$ closely parallels the outer contour of the radio lobe.

For our LRIS spectra, we placed a $1^{\prime \prime}$-wide slit through the quasar and components $f$ and $g$. In a total integration of 2 hours, only a faint continuum was seen for the SE component $g$, but a pair of faint emission lines is present in the spectrum of the NW component $f$. From their separation, these can be unambiguously identified as the [O II] $\lambda 3727$ doublet at a redshift of 0.927 . In the rest frame of the quasar $(z=1.05)$, the radial velocity difference is over $18000 \mathrm{~km} \mathrm{~s}^{-1}$. If this gas is in fact associated with $3 \mathrm{C} 212$, we require a mechanism that can eject it coherently at this velocity while keeping the internal velocity of the gas quite low. Note that the fact that we see a blueshift relative to the quasar frame is consistent with the NW radio lobe being the closer to us, as inferred from the radio jet being visible on that side.

\subsubsection{Discussion}

The coincidence of optical components $a, b$, and $c$ with peaks in the radio jet indicates that these features are most likely due to optical synchrotron emission. The plausibility of this conclusion is reinforced by our observation of similar features in 3C 2 and $3 \mathrm{C} 245$.

Understanding the nature of the aligned components that lie beyond the detected radio structure is much more difficult. Are $f$ and $g$ truly associated objects? The evidence in favor of association is entirely morphological, but if confirmed it could be very important for our understanding of FR II radio sources and the alignment effect in high-redshift radio galaxies and quasars. Another object that appears to have a red aligned component outside of its radio lobes is the $z \sim 0.7$ radio galaxy $3 C 441$. In this case, the red knot is only a couple of arc seconds beyond the radio lobe and strong emission lines are seen from the region just within the radio lobe (McCarthy et al. 1995; Lacy 1997). However, HST imaging

and optical and infrared spectroscopy make it clear that this is probably simply the result of the radio jet impacting a companion spiral galaxy, in which the enhanced line emission comes from an interaction of the spiral disk with the radio jet (Lacy 1997).

We will present a more detailed discussion of $3 \mathrm{C} 212$ in our upcoming paper, but we will briefly outline the nature of the problems and some possible options here.

The problem is this: some features of the morphologies of the optical components $f$ and $g$ suggest that their emission has resulted somehow from radio jet interaction with the ambient material, but the absence of any detected radio emission coincident with them 
makes it very difficult to understand how any plausible scenario of this type could work.

Object $g$ has a projected distance from the lobe "edge" (in the high-resolution $3.6 \mathrm{~cm}$ map) of $\sim 0$ ' 5 , corresponding to only $\sim 3 \mathrm{kpc}$. The narrow arclike wisps seen in the optical image could conceivably result from the bow shock expected to form at the head of a radio lobe propagating into the ambient medium. While the radio emission comes primarily from particles accelerated at the hotspot and the beam shock interior to the contact discontinuity, the broad bow shock itself is expected to be radio-quiet (Williams \& Gull 1985; Williams 1991). This view is supported by rotation measure (RM) observations of Cygnus $\mathrm{A}$, in which a radio-quiet bow shock associated with a hotspot is observed through a RM discontinuity the shocked gas induces in the projected radio lobe emission (Carilli, Perley, \& Dreher 1988). The projected distance from the bow shock (RM discontinuity) to hot spot is $\sim 3^{\prime \prime}$ or $\sim 3 \mathrm{kpc}$ for Cygnus A. The detection of the expected bow shock in Cyg A means that the association of the rest-frame UV emission in $g$ with a bow shock outside the radio lobe cannot be ruled out on considerations of distance from the lobe alone. While continuum and line emission in the wisps associated with $g$ could result from thermal emission from shock-heated gas, it would be difficult to explain the bulk of the emission from $g$ with this mechanism. Not only is the morphology of $g$ as a whole unlike that expected for a bow shock, its color is correct for a companion galaxy with an old stellar population and is much too red to be consistent with thermal emission from a shock.

Object $f$ is at a (projected) distance from the NW radio component of $\sim 20 \mathrm{kpc}$, or at least several times the apparent lobe width. This emission cannot plausibly result from a bow shock directly associated with the visible NW radio component. We briefly enumerate various possible explanations for $f$, along with some of their problems:

1) $f$ is an unassociated foreground object at $z=0.927$. In this case, the continuum is naturally explained as due to stars at the emission-line redshift, but the alignment and the apparent morphological connection is purely fortuitous.

2) $f$ is dominated by optical synchrotron emission associated with undetected radio synchrotron emission. Our upper limits to radio emission at the position of $f$ at 3.6 and 20 $\mathrm{cm}$ mean that the radio-optical spectral index must be flatter than 0.44 , which would be unusually low for an extended radio lobe. In the $z=0.162$ radio galaxy $3 \mathrm{C} 346$, Dey $\&$ van Breugel (1994) found an optical synchrotron knot associated with a radio knot with radio spectral index $\sim 0.4$. However, subsequent HST imaging established on the basis of the high resolution morphology that this optical synchrotron was probably from a jet rather than a hotspot (de Koff et al. 1997)

3) $f$ is dominated by inverse Compton scattering of microwave background photons 
associated with undetected or relic radio emission. This option would require a large total energy to be present in low-energy relativistic electrons (Lorentz factors of 10-100), which would be expected to emit synchrotron radiation around $1 \mathrm{MHz}$. While in general this explanation would be consistent with $f$ being a "relic" lobe from either a previous position of a precessing jet or a previous outburst of the radio source, in the sense that synchrotron losses would eliminate the higher-energy relativistic-electron population over time, the absence of a comparable level of inverse Compton emission from the current radio lobe is difficult to understand unless the relic lobe originally had far greater radio power.

4) The continuum in $f$ is due to jet-induced star formation from a previous position of the radio jet or a previous outburst. This interpretation deals with the lack of detection of radio emission, but it presents an additional problem: assuming that the stars share the velocity of the ionized gas (since the two components appear to be cospatial), how could the pre-stellar gas be accelerated to $\sim 18000 \mathrm{~km} \mathrm{~s}^{-1}$ while presumably retaining a velocity dispersion comparable to that we now see in the [O II] line? This last option seems very unlikely.

None of these options seems very satisfying at this time. We have recently obtained additional deep spectroscopy and imaging that may eliminate some of the possibilities or suggest others when fully analyzed. In the meantime, we can only emphasize that we regard the nature of the exterior aligned components in $3 \mathrm{C} 212$ as possibly one of the most important questions raised by this investigation.

\subsection{CR 217}

\subsubsection{Morphology}

We display in Fig. $7 A$ and $B$ our HST and $K^{\prime}$ images of the radio galaxy 3C 217. This is the only object in our sample without a detected radio core available in the literature. In this case we cannot align the radio and the optical frames by aligning the optical/infrared nucleus with the radio nucleus. Therefore, the radio lobes we indicate in Fig. 7 by the white cross and the white arrow show the approximate positions of the radio lobes, referenced to the measured optical core position (Pedelty et al. 1989). The lack of a radio core and the positional uncertainty make interpretation of the radio/optical morphologies somewhat ambiguous.

The optical image consists of an elongated galaxy with a very faint nuclear peak (the faintest in our sample); this peak is best seen in the inset to the magnified version of the HST image, shown in panel $C$. $D$ shows the same magnified version, slightly Gaussian 
smoothed. The near-infrared component appears round, and is centered on the optical peak. The seeing for the $K^{\prime}$ image was poorer than average at $0^{\prime \prime} 83$, however, and the infrared component is not well resolved. Nonetheless, subtracting the best PSF leaves some diffuse flux in the near-IR, and, for the $K$-band flux to be entirely due to the unresolved component seen in the HST image, it would have to be extremely red $(\alpha=2.9)$. There are also two semi-discrete components or companions (objects $a$ and $c$ in Fig. $7 A$ ). Both objects lie within the lowest envelope of extended emission in the HST image; the optical structure of the central component extends towards $a$ to the NE, while the object $c$ is elongated towards the SW side of the central component. Component $a$ is rounder, more compact and much redder than $c$. If these are included in a PA determination, the whole ensemble has a PA of $63^{\circ}$. They are oriented at close to the angle of the elongation of the rest of the galaxy; however, if they are excluded on isophotal cutoff or aperture considerations, the galaxy $\mathrm{PA}$ is $79^{\circ}$. Therefore the dominant morphology of the galaxy differs in orientation by $\sim 30^{\circ}$ from the PA between the radio lobes $\left(105^{\circ}\right)$ regardless of the details of what is included in the moment analysis. This galaxy is therefore globally not very well aligned with the radio lobe direction. There is an extension ( $b$ on Fig. 4 ) that is very linear at its highest isophotal level and has a PA (relative to its own center) that matches the radio lobe PA to within a degree. This extension is seen best in panel $C$; it points directly towards the eastern radio lobe with the radio-optical frame centering we have chosen, but its end is offset by 0.5 from the optical peak.

\subsubsection{Discussion}

This radio galaxy is relatively unaligned and has a faint optical nucleus. Nonetheless, the radio jet could conceivably be involved in the creation of the linear feature $b$, but only if the jet has moved through some fairly large angle $\left(\sim 20-30^{\circ}\right)$. The component $a$ is the reddest object among the nearby companions or semi-discrete components that we measured in any of our 10 sources, while component $c$ is among the bluest. This difference is unexpected from the appearance of the HST image, in which the two companions look fairly symmetric in distance and alignment with the rest of the galaxy. Assuming they are both associated, they must be quite different in nature; the color and morphology of $a$ is consistent with a companion galaxy, while the blue color of $c$ is similar to those of the companion objects 3C $196 d$ and $e$. Without a better radio map of this object, particularly one with a radio core detection, it is difficult to determine whether the radio activity is related to the morphology of extension $b$ or to the colors of companions $a$ and $c$. Certainly, $a$ resembles the many other red companion galaxies commonly seen around high- $z$ radio galaxies (Rigler et al. 1992). 


\section{6. $\quad 3 \mathrm{C} 237$}

\subsubsection{Morphology}

This radio galaxy is a compact steep spectrum source, with an angular size of $1^{\prime \prime} .3$, and an unresolved, flat-spectrum core (van Breugel et al. 1992). We display the HST and Keck $K^{\prime}$ images of the galaxy in Fig. $8 A$ and $B$; in panel $C$ we show a magnified version of the HST image, with the fairly symmetric radio lobes of van Breugel et al. marked with white crosses in an inset. As can be seen in Fig. $8 B$, the HST image shows a slightly elongated galaxy with several close companion galaxies. Our Keck $K^{\prime}$ band image shows a fairly round galaxy (axial ratio $b / a \sim 0.93$ ) whose center coincides with the optical peak to within 0.05. Despite its low ellipticity, the IR position angle is stable and aligned to the radio axis within $1^{\circ}$ at the lower contour levels. (We exclude the nearest discrete companion galaxy in our magnitude and moment analyses; it is at a distance of 3 ".9.)

The $15 \mathrm{GHz}$ VLA map of van Breugel et al. has an angular resolution $\sim 0^{\prime \prime} 15$, close to the resolution of our HST image. The optical image has a fairly dominant optical nucleus, with two lobes in a morphology roughly similar to that of the radio. If we align the radio and optical cores, the $\mathrm{E}$ lobe radio and optical peaks are fairly well aligned; the western radio lobe, however, is more extended and peaks past the optical emission.

\subsubsection{Discussion}

Despite the fairly close correspondence in scale and overall alignment between the radio and optical morphologies, there does not seem to be a close point-to-point coincidence. We therefore do not find for this object a compelling morphological case for the aligned, extended structure to be non-thermal radiation, as we do for $3 \mathrm{C} 2$. There is, however, an unresolved core in both the HST image and the van Breugel et al. (1992) radio map, and its radio-optical spectral index is 0.72 ; there is no problem with ascribing the optical core to synchrotron emission. The radio-optical spectral indices for the "peaks" of the extended material is in the range of 1.0-1.3 for the two lobes (depending on details of what flux is included in the estimate). Regardless of how much of the aligned emission is from synchrotron (or other non-thermal process) the kind of alignment shown is similar in some ways to what is seen in other small-scale radio sources, which tend to be closely aligned. The radio and optical structures are roughly co-spatial, as are those of $3 \mathrm{C} 368.3 \mathrm{C} 237$ is much redder, however, presuming the bulk of the near-IR is coming from the same origin as the optical flux, which is consistent with the close alignment of the near-infrared with the radio axis. It is less likely, therefore, to be dominated by nebular continuum, as is $3 \mathrm{C} 368$. 


\section{7. $3 \mathrm{C} 245$}

\subsubsection{Morphology}

We show in Fig. $9 A$ and $B$ our PSF-subtracted HST and Keck images of the quasar $3 \mathrm{C} 245$. This quasar had the brightest nucleus in our sample, making detection of underlying extension more difficult. We were also hampered in our $K^{\prime}$ imaging by an unfortunate period of poor seeing $\left(\sim 1^{\prime \prime} 3\right)$. Both images clearly resolve the companion galaxy $2 ! 3$ southeast of the quasar nucleus, studied by LeFèvre \& Hammer (1992). Their spectra show this to be a fairly normal elliptical, at $z=1.013$.

In the HST image, other than this elliptical galaxy, the highest surface brightness extension is a linear feature (containing components $a$ and $b$ ) to the west of the nucleus. If we align the quasar with the core of the 5-cm map of Laing (1989), this optical feature coincides exactly with the radio jet. The direct correspondence, even in the position and relative brightnesses of the radio and optical hotspots, is shown in Fig. $9 \mathrm{C}$. The inset at the lower right shows the central $3^{\prime \prime} \times 4^{\prime \prime}$ region of the PSF-subtracted quasar image, with contours from the 5-cm map of Laing (1989) superimposed. The radio map contours are offset vertically by $1^{\prime \prime}$ so that the optical image is easily visible for comparison.

In addition, there is low-surface-brightness extended flux to the east of the nucleus (including a peak at $d$ and extending down to the elliptical galaxy $e$ ) lying in the diffuse, extended eastern radio lobe of $3 \mathrm{C} 245$; the approximate center of this lobe is indicated by a cross (Laing 1989; Liu, Pooley, \& Riley 1992). The 5-cm map of Laing shows 3 faint peaks centered around the cross position; we see a corresponding 3 peaked structure in the optical emission. This structure could be due to nebular thermal continuum in our HST bandpass, if there is $\sim 10^{4} \mathrm{~K}$ ionized gas in this region. Unfortunately, our [O II] image (Fig. $9 D$; continuum subtracted) has insufficient signal-to-noise to show whether there is [O II] at a correspondingly low surface brightness level at this location. However, we do see resolved [O II] line emission extending for about $3^{\prime \prime}$ to the north, and at the location of the elliptical galaxy. In fact, the PA of the $[\mathrm{O} \mathrm{II}]$ emission line region is $\sim 3^{\circ}$ (measured in a $2^{\prime \prime}$ radius aperture, but excluding the elliptical); this orientation is within $2^{\circ}$ of perpendicular to the radio axis (as defined by the lobes; the jet direction is rotated by $10^{\circ}$ ).

\subsubsection{Analysis and Discussion}

We give fluxes for the components seen in the HST image in Table 5. The $K^{\prime}$ image has far too low resolution to allow us to get colors (or even useful limits) for the extensions 
near to the quasar, other than the bright elliptical.

The linear feature containing components $a$ and $b$ is certainly associated with the radio jet, and the small-scale correspondence between the radio and the optical makes it very likely that the optical emission is synchrotron radiation. From the Laing (1989) contour map, we find the approximate surface brightness ratio (at $6 \mathrm{~cm}$ ) of component $a$ to component $b$ is 2:1. In our optical image, $a$ has a surface brightness $\sim 3$ times that of $b$. From the plot of radio spectral index between 5 and $15 \mathrm{GHz}, \alpha_{15}^{5}$, of Liu et al. (1992), $a$ has $\alpha_{15}^{5} \sim 0.8$ and $b$ has $\alpha_{15}^{5} \sim 0.9$.

If there were no high-frequency turnover to the spectral energy distribution, the surface brightness ratio between the two components would increase by a factor of 3 from observations at $6 \mathrm{~cm}$ to $6700 \AA$. This is a greater increase in the surface brightness ratio than we observe; however, high-frequency turnovers are usual in synchrotron spectra where optical synchrotron has been observed.

\section{8. $3 \mathrm{C} 280$}

\subsubsection{Optical Morphology}

Under ground-based seeing conditions, the radio source $3 \mathrm{C} 280$ has been observed to be associated with an elongated galaxy that is well-aligned with the radio axis (within $1^{\circ}$ : McCarthy et al. 1987; within $20^{\circ}$ : Rigler et al. 1992). As seems to be the norm, with HST's resolution this elongated galaxy separates into several discrete components. However, even by the standard of previous HST images of high-redshift galaxies, 3C 280 is unusual. As shown in Fig. $10 \mathrm{~A}$ and $C$, the elongated, aligned UV component is resolved into a two-component central region (peaks $a$ and $b$ ), connected to a narrow structure $(c)$ by a semi-circular arc $(d)$. This arc is also clearly seen in the WFPC2 image of Best et al. (1996). The length of the arc is $\sim 1^{\prime \prime}$; at the redshift of the radio galaxy this corresponds to $\sim 6 \mathrm{kpc}$. We see no similar structures in the rest of our sample, nor are we aware any similar features found in any other high-redshift sources.

The objects $a, b$, and the peak of $c$ are collinear, at a PA of $80^{\circ}$, within $10^{\circ}$ of the radio axis, as determined from the lobe hotspots. (Unfortunately, we have no high resolution radio data available to use in this morphological comparison). Object $c$ is elongated almost perpendicularly to this axis. Object $a$ is unresolved, with an intrinsic width of $0{ }^{\prime} 14$ on the HST image, and presumably represents the position of the active nucleus and thus the radio core. 
The arc $d$ can also be seen in our continuum-subtracted CFHT [O II] image shown in Fig. $10 D$ ); the arc is best seen in the inset. (This image had an initial resolution of 0 !n, and its resolution was improved by deconvolution with the Lucy restoration algorithm and subsequent reconvolution with a Gaussian).

Components $c$ and $d$ appear to be edge-brightened, along the width of each feature, and well aligned with the radio jet direction as can be seen clearly in Fig. 10C. The brightened region in the edge of the arc has about twice the surface brightness of the rest of the curve and extends out to a PA of about $30^{\circ}$ from the radio axis, or $40^{\circ}$ from the optical axis. This is consistent with the probable opening angle of the quasar opening cone derived by Barthel (1989) and Saikia \& Kulkarni (1995).

\subsubsection{The Near-Infrared Morphology}

In the near-infrared (rest-frame $1 \mu \mathrm{m}$ ) the galaxy is fairly round and resolved with no obvious sign of the discrete aligned components seen at rest-frame $0.33 \mu \mathrm{m}$. Its center is coincident with the brightest HST peak, the unresolved component $a$, to within 0.06. (The $K^{\prime}$ image has an intrinsic FWHM of $\sim 0$ ' 58 ).

We fit the elliptical galaxy using the STSDAS routine ellipse, which fits elliptical isophotes using the iterative algorithm of Jedrzejewski (1987). If we let the PA, ellipticity, and center vary, we obtain the profile shown in Fig. 16. This is well fit by a de Vaucouleurs $r^{\frac{1}{4}}$-law profile between 0.5 and $1^{\prime \prime} 46$ (the radial region in which the elliptical isophotes were good fits, and outside of the inner, seeing-flattened region). The effective radius is $r_{e}=0$ "' 86 $\approx 5 \mathrm{kpc}$. The $\mathrm{PA}$ of the elliptical isophotes varies from about $65^{\circ}$ to $80^{\circ}$ and the axial ratio $(b / a)$ varies from about 0.87 to 0.91 in this radial region. We show the PA as a function of the semi-major axis in the lower panel of Fig. 16. The PAs derived from these elliptical isophotal fits are consistent with the results of the moment analysis discussed in 6.2 ; the dependence on radius translates roughly to the isophotal cutoff and size of the apertures used in the formal moment analysis. We find that the $K^{\prime}$ light is aligned with the radio PA within $25^{\circ}$ at all isophotal levels, and more closely with the axis defined by the optical components $a$ and $b$.

To investigate the contributions of the discrete aligned components, we fix the axial ratio $(b / a=0.9)$ and $\mathrm{PA}\left(\sim 70^{\circ}\right)$, derive an intensity profile, and use this to make a 2-dimensional model of the galaxy. We subtract the model from the infrared image of the radio galaxy, varying the center and scaling slightly to reduce the residuals. There is evidence from the best-fit residual for some flux at the position of the component $c$ 
in the HST image. However, a discrete contribution from this component cannot have caused the elongations along $\mathrm{PA} \sim 70-80^{\circ}$ that we observe, since the elliptical isophotal fits have relatively consistent PAs and axial ratios through all the isophotes. To further verify this, we subtract the galaxy's azimuthal average from itself and find the obvious bimodal, large-scale residuals (along the axis defined by the best PA of the elliptical isophotes), that are consistent with a non-circular morphology. (We also see again the extra flux at the position of the component $c$ ). In addition, to make sure this elongation we see is not the result of an elongation in the observed PSF, we have checked the position angle and axial ratio of the star on the $K^{\prime}$ frame and seen no preferential elongation. The stellar image is very circular; position angle determinations vary randomly over various apertures and isophotes. This best-fit elliptical galaxy has a total $K^{\prime}$ magnitude of 17.0 within an aperture of $8^{\prime \prime}$, consistent with the $17.1 \pm 0.3 \mathrm{~K}$ magnitude found by Dunlop \& Peacock (1993).

\subsubsection{Photometry}

We now use the elliptical model to scale and subtract from the smoothed version of the HST image to try to determine an optical-infrared color for this extended elliptical component. We smooth the HST image with a Gaussian until the star that falls in both the $K$ frame and the HST frame matches in FWHM; we then determine the colors of any shared components. We subtract off the $K$ elliptical galaxy model in steps until we have started to over-subtract the wings; this defines the upper limit to how much of the elliptical flux could be present in the HST $\lambda_{0}=3300 \AA$ image. We make this estimate from the unsmoothed HST image as well, as the wings of the elliptical outside of $r=0^{\prime \prime} 6$ should not be affected by the seeing, and the unsmoothed image will provide better contrast against the nucleus. We find consistently from the smoothed and unsmoothed images that the ratio of the HST to $K$ extended envelope is between 0.2 and 0.4 , with 0.3 providing the best apparent fit. Assuming all this flux were stellar, from a single generation of stars, and without significant reddening, comparison with Bruzual-Charlot (1993) models shows that this color would fit a population with an age of between 3 and $4 \mathrm{Gyr}$, comparable to the age of the old stellar population found for the $z \sim 1$ radio galaxy 3C 65 (Lacy et al. 1995; Stockton, Kellogg, \& Ridgway 1995)

We use these elliptical-subtracted HST images to obtain photometry of the aligned components alone, using isophotal cutoff levels of about 3-4 $\sigma_{s k y}$. (This level is comparable to the lowest grey level in the upper-right inset in Fig. $10 \mathrm{C}$ ). We give the results of this photometry in Table 5 . 


\subsubsection{Spectroscopy}

Although we have used essentially line-free filter bandpasses to maximize contributions from stellar continuum radiation, our bands could still be dominated by emission from an ionized gas. For our HST imaging, which covers the rest-frame region near $3300 \AA$, the most likely potential contributors are thermal continuum emission processes (i.e., free-free, free bound, and 2-photon emission) and the [Ne V] $\lambda 3346,3426$ lines. For 3C 280, we see in our HST image a relatively fainter echo of the [O II] emission-line morphology associated with the eastern radio lobe (Rigler et al. 1992; Fig. 10D). This similarity increases the likelihood that at least some of the structure we see in our HST image may be dominated by gaseous emission.

We have recently obtained deep Keck LRIS spectroscopy of $3 \mathrm{C} 280$, which will be reported in detail later (Ridgway \& Stockton 1997a). Here we restrict ourselves to an evaluation of the ionized gas contribution to our HST images. We consider three regions: the central region, comprising $a$ and $b$ in $10 A$, object $c$, and the faint emission just $N$ of the $E$ radio lobe. We have evaluated the contribution of the nebular thermal emission by scaling a model at $10^{4} \mathrm{~K}$ to the observed $\mathrm{H} \delta$ line, and we have measured directly the equivalent widths of the [Ne V], O III $\lambda 3133$, and He II $\lambda 3203$ lines. We find that the emission lines in all regions account for $5 \%$ or less of the total flux in the $\mathrm{F} 622 \mathrm{~W}$ filter. The thermal continuum from the ionized gas is more significant: it comprises about $20 \%$ of the total flux from the central region and about $50 \%$ of the total from object $c$ and the optical features in the $\mathrm{E}$ radio lobe. In all cases, we clearly seem to have a continuum component beyond that from the nebular thermal emission. Although we cannot isolate a spectrum of the arc $d$ alone in our data, its [O II] $\lambda 3727$ to continuum ratio is very similar to that of $c$, and we assume that our conclusions for $c$ apply to $d$ as well.

\subsubsection{Discussion}

$3 \mathrm{C} 280$ is one of the more classical cases of the alignment effect in our sample; i.e., one in which a number of discrete components are quite linearly arranged, close to the presumed radio jet direction. We know that this aligned morphology is traced by both the line and continuum emission, and that there is probably a significant nebular thermal continuum contribution to the main peak $(a+b)$ and a larger, possibly dominant, one to $c$ and $d$, as well as to the extended material around the eastern radio lobe.

One of the more intriguing and unusual features of its high-resolution morphology is the arc between components $b$ and $c$. What are possible origins for this curved structure, 
along with the rest of the aligned morphology?

First, we consider the possibility that it is a gravitational arc; i.e., that it is either a distant object lensed by some mass associated with $3 \mathrm{C} 280$, or a component of $3 \mathrm{C} 280$ lensed by an intervening mass. It is, however, extremely unlikely that the arc $(d)$ is a gravitational image of a distant object for several reasons: first, the presence of line emission tracing the arc morphology at the redshift of the radio galaxy; second, the edge brightening, plausibly associated with a cone of quasar illumination approximately centered on the radio axis; third, the fact that the $K$ elliptical galaxy is centered at the peak of the optical emission (component $a$ ) rather than somewhere near the center of curvature of the arc, and that there is no other likely candidate for the lensing mass. The second possibility, that of an intervening lensing mass gravitationally lensing some feature associated with $3 \mathrm{C} 280$, would remove the first objection, but not the second and third. Indeed, the lack of detection of a plausible lens mass becomes more acute as it is moved to lower redshifts.

Another interpretation is that the arc could be a tidal tail from $c$. A simulation by Mihos (1995) of the detectability with WFPC2 of tidal tails resulting from disk galaxy mergers indicate that from normal spirals, tidal merger remnants such as tails would only be visible at $z \sim 1$ for about $200 \mathrm{Myr}$ after the initial interaction. (Mihos assumes a total exposure time with the WFC of $10,000 \mathrm{~s}$, similar to our 3C 280 exposure of $8800 \mathrm{~s}$ ). While the arc in $3 \mathrm{C} 280$ is not only detected, but could have been detected in a considerably shorter exposure, its relatively high surface brightness is almost certainly largely due to enhancement from external ionization and scattering. In this view, the alignment of $c$ and $d$ with the radio axis would be enhanced as a result of their lying within the ionization cone of the active nucleus.

However, because of the close linear alignment of the peaks of $a, b$, and $c$, this, like $3 \mathrm{C} 324$, is another case of an aligned structure that is not consistent with scattering and ionization filling an entire ionization cone. While the edge-brightened regions of $d$ and $c$ do fill a fairly large angle $\left(\sim 30^{\circ}\right)$, the peaks of $a, b$, and $c$ are aligned within degree or so, and raise the possibility that this axis may correspond to the jet direction, and that jet/cloud interactions are affecting the morphology. Jet/cloud interactions and related shocks are considered a possible cause of the closely aligned components in 3C 368 (Stockton et al. (1996); Clark et al. (1997), which also have large thermal continuum contributions.

In the near-infrared, the morphology and colors are consistent with an elliptical galaxy. However, there may also be a contribution from an unresolved core, and the alignment of the elliptical isophotes with the radio axis suggests that there is some contribution from an "aligned" component. The aligned components $c$ and $d$ would still be resolved from the central component $a$ at the ground-based resolution, but the component $b$ would not be. 
The infrared morphology could not track the optical exactly, as the central component is much rounder than the combined $a$ and $b$ component in the HST image when smoothed to ground-based resolution. However, the PA of the elliptical isophotes is close to the axis defined by $a$ and $b$, and the alignment of the observed infrared morphology could be most easily explained by the addition of the unresolved $b$ to the red resolved, round component that is much more dominant at $K$ than in the optical. A second possibility is the contribution of some new red aligned component, perhaps a minor synchrotron component with a steep enough spectrum to be unobservable in the blue, that is sufficient to align the infrared isophotes. The third possibility is that even at high-resolution the infrared isophotes would be aligned, and the major axis of a nearly round "elliptical galaxy" is aligned with the radio axis. This question is interesting in light of our finding in several of our radio galaxies that the infrared elliptical isophotes of the central components are aligned with the radio axis; indeed, Dunlop \& Peacock (1993) claim for their $z \sim 1$ sample that the infrared morphologies are better aligned than the optical. In this particular example, the optical and infrared alignments match well, and the simplest explanation is that the infrared alignment comes from the unresolved contribution of $b$.

There appears to be a cluster around $3 \mathrm{C} 280$; we see some objects which seem to have [O II] emission at the redshift of the radio galaxy. There is also evidence for a cluster in the detection of extended X-ray emission (Worral et al. 1994; Crawford \& Fabian 1995).

\section{9. $\quad 3$ CR 289}

We show in Fig. $11 A$ and $B$ our HST and Keck $K^{\prime}$ images of the radio galaxy 3C 289 . This galaxy is unlike the rest of the galaxies in our sample, in that it shows some discrete, elongated structure within the central few arcseconds that is severely misaligned with the radio axis $\left(\triangle \mathrm{PA}=75^{\circ}\right)$. The components $a, b$ and the central component are collinear, at a PA of $0^{\circ}$. This collinear feature looks superficially similar to the "chain" of components seen in $3 \mathrm{C} 212$, but that feature lies directly on the radio axis. At higher isophotal levels the central component has a PA within $20^{\circ}$ of the radio axis; PSF subtraction shows most of the central component to be unresolved, but with a small bright asymmetry as well as the obvious low surface brightness material. Using azimuthal averaging to remove the asymmetric material in a manner similar to that used for $3 \mathrm{C} 2$. we derive a flux for this extended low surface brightness material and give this value in Table 5 under the label

"elliptical". (This is the flux of the symmetric optical material, minus that of the unresolved core).

In the infrared, the galaxy looks morphologically similar to its appearance in the 
optical. There is a dominant central "elliptical galaxy" component; outside of the seeing radius, the isophotes are well fit by a de Vaucouleurs profile with $r_{e}=1^{\prime \prime} 2 \pm 0^{\prime \prime} 3 \sim 7 \mathrm{kpc}$. The PA is within $10^{\circ}$ of the PA of the elliptical component in the optical $\left(30^{\circ}\right.$ from the radio axis). These PAs are close, as well, to that of extension $c$. The [O II] image shows a bright extension that matches $c$ well; this is then a case in which the emission line morphology is better aligned with the radio axis than is the optical or infrared continuum. In the near infrared, the elliptical central component dominates the components $a, b$, and $d$, though $a$ and $d$ are also detected in the infrared. The optical and infrared fluxes for the symmetric material are estimated by azimuthally averaging the images and therefore removing any non-symmetric components, and are given in Table 5 with the label "elliptical". The optical flux is corrected for the unresolved nucleus, while the infrared value is not corrected for any unresolved component contribution. We have only a limit to the infrared nuclear component, but the contribution should be small (at most 20\%). Component $d$ is unresolved on the HST image with FWHM $\sim 0^{\prime}{ }^{\prime} 15$, while $a$ is marginal and $b$ is clearly resolved with FWHM $\sim 0.3$. Component $d$ could be a faint, red foreground star, while $a$ and $b$ are potentially companions or components of $3 \mathrm{C} 289$.

\subsection{0. $3 \mathrm{C} 336$}

\subsubsection{Morphology}

We show in Fig. $12 A$ and $B$ our HST and Keck $K^{\prime}$ images of the quasar 3C 336; this is the source with the largest angular size in the radio of any in our sample $\left(28^{\prime \prime}\right)$. The radio structure of this quasar has been well-studied by Bridle et al. (1994). We give the directions toward each radio lobe as arrows in panel $A$. In the HST image, we have resolved low-surface brightness extension near to the nucleus; this can be seen well in panel $C$ where we display a magnified version of the PSF-subtracted HST image, slightly Gaussian-smoothed. Also seen are PSF subtraction residuals: this quasar had the worst match between PSF and nucleus, particularly in the diffraction spikes. (We chose the subtraction scaling we display here by requiring approximate monotonicity in regions which exclude these diffraction spike residuals.)

Bridle et al. detect a radio jet to the SW; the knot nearest to the nucleus in this jet is at a radius of 0.97 and $\mathrm{PA}-162^{\circ}$. The brightest extension we have resolved around 3C 336 in the HST image is at a similar radius, on the jet-side of the nucleus, but it is not coincident with the radio knot. In fact, this radio knot position corresponds to a break in the extension to the south of the quasar, to the west of extension $a$, giving a "channel" effect similar to that seen in Cygnus A (Stockton, Ridgway \& Lilly 1994). 
We display in panel $D$ the PSF-subtracted, continuum-subtracted [O II] image. The emission line region is very asymmetric with respect to the nucleus, and it has low surface brightness structure to the northwest that is approximately perpendicular to the radio axis. There are relative peaks in the emission at the positions of the continuum components $b$ and $a$. The [O II] peak near $a$ is at a radius of $1^{\prime \prime} 0$ and PA $-172^{\circ}$; though the [O II] is at lower resolution than both the HST image and the radio map, this [O II] knot seems to be better associated with the peak $a$ in the HST image $\left(\mathrm{PA} \sim-176^{\circ}\right.$ ) than with the position of the radio jet knot.

\subsubsection{Discussion}

We see no evidence for optical synchrotron emission in this quasar. The large scale size of the radio source could mean that the radio axis is closer to the plane of the sky than it is for the other quasars in our sample, preventing our detection of beamed optical synchrotron components like those we see in at least three of our other quasars.

We cannot be certain from our imaging data alone that the extensions we see are necessarily associated with the quasar; the spectrum of the quasar shows 6 metal absorption line systems, indicating the presence of several foreground objects along the line of sight (Steidel et al. 1997). The field of this quasar has been quite well studied from the ground. Bremer et al. (1992) made a spectroscopic study of the [O II] extension and detected extended line emission out to $5^{\prime \prime}$ north and $3^{\prime \prime}$ south. This is qualitatively consistent with our [O II] image. Hintzen, Romanishin, \& Valdes (1991) found an over-density of field objects, and suggested that 3C 336 was likely in a rich cluster. A spectroscopic survey of the field by Steidel \& Dickinson (1992) identified a galaxy 5"7 to the southwest of the quasar as responsible for the $z=0.4722$ absorption line system. Very recently, Steidel et al. (1997) have completed a comprehensive survey of galaxies in the 3C 336 field, using HST WFPC2 and ground-based imaging, and Keck spectroscopy. While their main interest is in identifying galaxies responsible for the multiple absorption-line systems seen in the spectrum of the quasar, they find that the objects we have labelled $a, b$, and $c$ have, respectively, redshifts of $0.927,0.931$, and 0.928 , showing a clear association with the quasar at a redshift of 0.927 . From both the morphology and the close agreement in redshift, it appears likely that $a$ and $c$ are either features in the quasar host galaxy or companions in the process of merging with it. Object $d$ has a redshift of 0.892 and is responsible for one of the absorption-line systems. If we ignore $d$ (as a confirmed foreground galaxy) and $b$ (as a discrete companion), the remaining objects $a$ and $c$, which appear to be embedded in diffuse surrounding emission and to be at essentially the same velocity as the quasar, 
are fairly well aligned with the radio axis. In addition to $a, b$, and $c$, Steidel et al. find 6 galaxies with redshifts close to that of the quasar within a radius of $50^{\prime \prime}$, demonstrating that the quasar is in a significant group or cluster.

\section{Properties of the Sample}

Here we discuss the results from our isophotal and annular magnitude and moment analysis of all the objects in our sample. To determine the properties of the extended material, we first exclude known or likely companions. We recognize from the start that it will be difficult with this small sample to judge whether the radio galaxy and quasar subsamples could be drawn from the same population, particularly for properties such as the total magnitude, color, and $\triangle \mathrm{PA}$, since the intrinsic dispersion in the properties in each subsample is large.

\subsection{Magnitudes of the Extended Material}

If radio galaxies are unbeamed versions of quasars, the extensions of both classes (if they are host galaxies) should have about the same total luminosities. The photometry for the extended material in the HST images is given in Tables 7 and 8 , and for the near-infrared images in Tables 9 and 10 (all values are normalized to redshift 1 ). We give in these tables both isophotal flux densities (down to a common limiting isophotal level) and flux densities within an aperture, excluding a central PSF-subtraction region for both the quasars and for the radio galaxies, as well as with no central exclusion for the radio galaxies. In addition, for some of the quasars with sufficient extended material, we give flux densities with the central subtraction region interpolated with a low-order polynomial. The photometry generally excludes spectroscopically determined foreground objects; details of the calculations are given in Appendix A.

For inter-comparison, we use the flux densities that exclude the region of PSF subtraction (generally the inner $0.45 \sim 2.7 \mathrm{kpc}$ radius for the HST images, and the inner 0 '.85 $\sim 5 \mathrm{kpc}$ for the near-infrared images), and we also exclude values below the lowest common normalized isophote above sky noise.

For the WFPC2 data, we find that the average flux density (all values are in units of $10^{-20} \mathrm{ergs} \mathrm{s}^{-1} \mathrm{~cm}^{-2} \AA^{-1}$ ) in the annular aperture $0.45-3.75$ above our fiducial isophotal cutoff level is $206 \pm 137$ for the quasars, and $124 \pm 95$ for the radio galaxies, where the errors given represent the dispersion in the flux over each set of 5 objects. For the total annular 
flux density, without application of the isophotal cutoff level, the quasars have an average value of $398 \pm 116$, and the radio galaxies have an average flux density of $308 \pm 96$.

The optical flux densities of the two subsamples therefore match well within the observed dispersion, despite the fact that we see a beamed optical synchrotron component in the quasars but not in the radio galaxies. We can estimate this contribution by explicitly subtracting the flux from the probable optical synchrotron components $3 \mathrm{C} 2 a, 3 \mathrm{C} 245 a, b$, and $3 \mathrm{C} 212 a, b, c$, giving a mean total annular flux density for the quasars of $360.6 \pm 116$. This change is negligible within the error.

In the $K^{\prime}$ data, we find for the quasars, the average total isophotal flux density is $168 \pm 156$, and the average total annular flux density is $253 \pm 168$; for the radio galaxies, the average isophotal flux density $198 \pm 133$, and the average total annular flux density is $302 \pm 169$. (The synchrotron contribution is minor, and resolved in the infrared only in $3 \mathrm{C}$ 2.) Our results are consistent with the unification hypothesis of AGNs, in that we see no evidence for a significant luminosity difference between the quasars and the radio galaxies for extended material that plausibly could be identified with a host galaxy. The intrinsic dispersion in this small sample is large, however.

As the optical quasar extensions seem generally to have larger synchrotron contributions, the $K^{\prime}$ fluxes of the extensions are more appropriate to use in considering how these quasar extensions compare to earlier quasar host and radio galaxy determinations. First, we should try to correct, if possible, for flux obscured in the PSF-subtraction region. The average flux added to the quasar total fluxes by interpolation over the central region is only 34.7 , and the largest correction is that for $3 \mathrm{C} 2$, whose inner region is affected by the high surface brightness northern synchrotron component. More relevant is the comparison of the total $K^{\prime}$ fluxes for the radio galaxies with and without exclusion of the nuclear region. We find the average flux difference is $194 \pm 72$, which corresponds to a difference of 0.55 mag. If we then apply this average correction to the quasar hosts, we can estimate the average $K^{\prime}$ magnitude to be 17.4. Both the mean and the dispersion of the quasar hosts are consistent with the $K-z$ relation for radio galaxies (e.g., Lilly 1989).

We can further attempt to estimate how the radio galaxies and quasar hosts compare with an $L_{K}^{*}$ galaxy at the present epoch. Mobasher, Sharples, \& Ellis (1993) find $M_{K}^{*}=-25.1$ (for $H_{0}=50$ ), independent of galaxy type. Converting to our assumed $H_{0}=75 \mathrm{~km} \mathrm{~s}^{-1} \mathrm{Mpc}^{-1}$ and placing such a galaxy at our fiducial redshift of $z=1$, we find that it would have a flux density of 176 (in our standard units of $10^{-20} \mathrm{ergs} \mathrm{cm}^{-2}$ $\mathrm{s}^{-1} \AA^{-1}$ ) in the redshifted $K$ band. To determine the flux density in our observed $K^{\prime}$ band, we must include the portion of the usual $k$-correction due to the difference of the galaxy SED between the rest-frame and observed bandpasses (we have already included the 
$(1+z)^{-1}$ bandwidth factor in the above calculation). The spectral index between $1 \mu \mathrm{m}$ and $2.5 \mu \mathrm{m}$ differs very little among different stellar populations with ages greater than $\sim 1$ Gyr; we assume a 4-Gyr-old population, consistent with the color we find for the elliptical component of 3C 280, our best studied case. From a Bruzual-Charlot (1993) model, we find a flux-density ratio of 4.58 , so our $L^{*}$ galaxy at $z=1$ would have an observed $K^{\prime}$ flux density of 807 , where we have ignored any evolutionary corrections. Taking our actual observed average values for our sample of quasar hosts and radio galaxies, and correcting for both the average $0.55 \mathrm{mag}$ loss in the masked central region and an estimated $0.2 \mathrm{mag}$ aperture correction, we find formal corrected flux densities of $506 \pm 183$ for the quasar hosts and $604 \pm 184$ for the radio galaxies. Thus we find these galaxies to be roughly $L^{*}$ or a little fainter. Given potential systematic uncertainties in this result and the apparent range in host-galaxy properties both in our sample and in the low-redshift samples, it would be premature to claim that our result is seriously at variance with the finding that low-redshift QSO hosts average 0.4-0.7 mag brighter than $L^{*}$ (McLeod \& Rieke 1994b,1995; Bahcall et al. 1997).

\subsection{Morphologies and Alignments of the Extended Material}

We might also expect the radio galaxy UV continuum "alignment effect" to show itself in the quasars in some form, but at a reduced amplitude relative to that seen in the radio galaxies because of projection effects. The amplitude of the aligned component in quasars, for an opening angle of $45^{\circ}$, should be about half of that seen in radio galaxies. Using techniques similar to the magnitude derivation (excluding the central region from both the radio galaxies and the quasars, and using the flux only to normalized comparable surface brightness levels), we derive $\Delta$ PA (Radio PA $-0.3 \mu \mathrm{m}$ PA, and Radio PA $-1 \mu \mathrm{m}$ PA). We use a simple, formal moment analysis as discussed in Appendix A.2. While it is straightforward to plot the dependence of the axial ratio and PA on the isophotal cutoff and aperture, the difficult (and subjective) part of this procedure is in choosing what aperture and isophotal cutoff (and resultant position angle) one will select. We display in Fig. 17 an example of the variation we see in our moment analysis. We plot the isophotal cutoff on the $x$ axis and the PA on the $y$ axis; the size of the circular points are proportional to the size of the aperture used in the moment determination.

To choose one position angle to represent the overall alignment of a complex source may not be possible in many cases. We thus endeavor to understand any dependence of position angle on the aperture and isophotal cutoff on a case-by-case basis. Adopting a single aperture and cutoff level is perhaps the most objective criterion, but it will also 
introduce a greater level of dispersion into the measurement of alignment; moments will still be skewed by included foreground or unrelated companion objects, and unexcluded noisy sky pixels. We have a small sample, and cannot afford this increase in dispersion.

In many cases, there is a "dominant" position angle associated with an isophotal-cutoff vs. PA plot. For the HST images, once the isophotal cutoff is above the value chosen for our magnitude analysis, the sky noise contribution is much reduced. This can be seen in these plots as a stabilization of the position angle with respect to changes in isophotal cutoff or aperture size. After such stabilization, the position angle in some cases will remain quite constant with aperture size and increasing isophotal cutoff. In these cases, the dominant position angle is fairly clear, and we adopt this value, generally an average over a range in which the position angle is relatively flat. In other cases, the determination is not so simple: discrete companions may skew the position angle at larger apertures or lower isophotal levels, a primary object may itself have different dominant position angles at low and high isophotal levels, and, in at least one case, once above the sky noise the isophotes twist smoothly, and there does not appear to be any dominant position angle. We adopt an aperture criterion similar to that of Dunlop \& Peacock (1993) to remove ambiguity in a few cases; in the others, we record both a "high" and "low" isophotal position angle; our "low" isophotal cutoff level is generally the same as that used for our magnitude calculation, and our "high" level is about 5 times this. The existence of this "high" and "low" position angle is particularly important in light of our need to compare results between the radio galaxies and quasars; in some cases, the removal of the center of the source removes any "high" isophotal level.

The $K^{\prime}$ data are of lower resolution, and the radio galaxies have fairly round central components. We find that in most cases the variation in position angle is related to inclusion or exclusion of companions. We therefore once again use an aperture criterion similar to that of Dunlop \& Peacock (1993), and a moderate isophotal cutoff level of about 2 times our magnitude isophotal cutoff level for $K^{\prime}$.

In addition, we are often interested in the behavior of the most central component (or extension) alone. For example, we have seen in $\S 5.8$ that the near-infrared central elliptical component in $3 \mathrm{C} 280$ seems to have elliptical isophotes that are aligned preferentially with the radio axis. A similar moment analysis of the central component will be clearer if we exclude the bright red companion. For this reason, for the infrared images, we have also calculated position angles excluding all companions that we interpret as discrete from our high-resolution HST images or that we know are unrelated from spectroscopy.

In Figs. 18 and 19, we give histograms of the results of our alignment effect analysis for the HST images for the complete sample, and for the two subsamples. In Fig. 20 we 
show histograms of the alignments derived from the near-infrared imaging for the samples; the values shown are for the central component, excluding companions. The radio axis of $3 \mathrm{C} 175.1$ is bent; we adopted the average PA of the two lobes as the radio PA for this histogram.

Application of a formal alignment analysis finds alignment in some of the galaxies and in several of the quasars, and we find no significant difference between the two samples from this analysis, for either the near-infrared or optical alignments.

It is clear, however, from inspecting the details of the moment analyses, that much of the alignment detected in the quasar subsample comes from discrete components that we have identified as high-surface-brightness optical synchrotron emission. The quasars $3 \mathrm{C} 2,3 \mathrm{C} 212$, and $3 \mathrm{C} 245$ all show direct correspondences between the optical and radio peaks. The alignment detected in the radio galaxy subsample, however, is qualitatively different. In 3C 280 and 3C 237, the most aligned galaxies, most of the extended material and components are aligned in both the optical and near-infrared without having a detailed optical-radio coincidence.

In the rest-frame $\sim 1 \mu \mathrm{m}$ images, we find that four of the radio galaxies are clearly resolved. The three with sufficient resolution to attempt a profile analysis (3C 175.1, 3C 280, and $3 \mathrm{C} 289$ ) are well fit by de Vaucouleurs $\mathrm{r}^{\frac{1}{4}}$-law profiles with effective radii varying from 5 to $7 \mathrm{kpc}$. The two radio galaxies which show close alignment in the optical (3C 237 and $3 \mathrm{C} 280$ ) have IR central components whose major axes are aligned with the optical/radio axis, presumably from unresolved contributions from the aligned material (rather than from a global alignment of an old stellar population). The presence of highly-structured, often aligned components in the rest-frame UV, coupled with a dominant elliptical component at $1 \mu \mathrm{m}$ is entirely consistent with the view that most powerful $z \sim 1$ radio galaxies result from nuclear activity in already mature stellar systems (the "old-galaxy hypothesis;" e.g., Rigler et al. 1992). This view is also supported by the colors of the elliptical components (see $\S 6.3)$.

\subsection{Colors of Components}

In Table 5, we give colors in terms of the optical-near-infrared spectral index $\alpha$ for a number of the identifiable components around both the quasars and the radio galaxies. We divide these objects and components into somewhat arbitrary categories: "discrete," consisting primarily of totally resolved companion objects and synchrotron components, "nebulous," consisting of extended components, such as central radio galaxy components 
and the nebulosities surrounding the quasars, and "nuclear," the quasar nuclei with all extended material subtracted. Some components (such as $d$ of $3 \mathrm{C} 212$ ) we include in both the discrete and nebulous classes. In Fig. 21, we show histograms of these $\alpha$ values for each of the categories. The top panel gives the values for the quasar nuclei (minus any extension); the middle panel gives the values for discrete companions, while the lower panel represents primarily nebulous material. Once again, the objects that are intermediate between these two classes are included in both middle and lower histograms. We note certain characteristics that are common to a group of components. The most obvious of these is that the "discrete" components (consisting primarily of totally resolved companion objects and synchrotron components) are bluer in color than the "nebulous" components, which include a number of the radio galaxies themselves as well as large scale nebulosities surrounding the quasars. In addition, all of the components that surround a particular quasar are of the same color or redder than the nucleus of the quasar in the field.

This supports (in a general sense) the idea that the nebulosities surrounding high- $z$ quasars are dominated by stars, and not by scattered quasar light. For comparison, a Bruzual-Charlot model of a stellar population with a Scalo IMF that has aged 4 Gyr since a delta-function starburst has an optical spectral index (from $0.33 \mu \mathrm{m}$ to $1 \mu \mathrm{m}$ ) $\alpha=2.56$.

What actual evidence is there for stars in these nebulous hosts? In general, "proof" of the existence of stars is scarce in high- $z$ radio sources. In $3 \mathrm{C} 65$, a red, unaligned $z \sim 1.2$ radio galaxy, and in 53W091, a low radio luminosity $z \sim 1.5$ radio galaxy, Keck LRIS spectroscopy has identified stellar absorption lines directly and found ages $\sim 4 \mathrm{Gyr}$ (Stockton et al. 1995; Dunlop et al. 1996). Additionally, $4000 \AA$ breaks have been detected in a number of lower signal-to-noise spectra of radio galaxies (Cimatti et al. 1993). In our sample, we have only a few broad-band colors; we can only say that the colors of the quasar nebulosities are inconsistent with simple scattering models and broadly consistent with a stellar origin. We find also that in those radio galaxies where we can identify a common "elliptical" component in the observed infrared and HST images, the colors of this component are consistent with those expected for a 3-4 Gyr Bruzual-Charlot model.

\subsection{Clusters and Companions}

The detailed clustering analysis of these fields will be presented elsewhere (Ridgway \& Stockton 1997b); here we will simply mention the best candidates (normally within $2-3^{\prime \prime}$ ) for companion galaxies physically close to the active galaxies in this sample, and a few cases in which there is obvious evidence for a cluster. 
The only spectroscopically-confirmed close companions to objects in our sample are $3 \mathrm{C} 245 e$ and $3 \mathrm{C} 336 a, b, c$. The bright companion to 3C 245 is an elliptical with a $3000 \mathrm{~km}$ $\mathrm{s}^{-1}$ velocity difference from the quasar redshift (LeFèvre \& Hammer 1992). Its color index is $\alpha_{K, R} \sim 2.6$ and its effective radius is $4.5 \mathrm{kpc}$. Of the objects associated with 3C 336, $a$ and $c$ may be part of the host galaxy, but $b$ appears to be a companion with a velocity difference of $\sim 600 \mathrm{~km} \mathrm{~s}^{-1}$ (Steidel et al. 1997).

Any other companion identification we must base solely on morphology and colors. 3C 2 has component $c$ which is resolved and as previously discussed has colors of an old stellar population if it is at the redshift of the quasar. 3C 217 includes component $a$, which is red and resolved, with $\alpha_{K, R} \sim 3.0$. 3C 289 has $d$, also resolved, and red with $\alpha_{K, R} \sim 2.5$. Not quite resolved from the $3 \mathrm{C} 196$ nuclear region is $a$, a blue component $\left(\alpha_{K, R} \sim 1.3\right)$, which could be part of the host or other associated material, perhaps with contributions from scattered light or thermal continuum; it is less likely to be a discrete companion.

Around $3 \mathrm{C} 212$, there is an over-density of objects within $10^{\prime \prime}$, which could be due to a cluster at the redshift of the quasar. Around 3C 280, we have more definite signs of a cluster, in that we detect a number of objects in a continuum-subtracted [O II] image. We will present spectroscopic follow-up to these objects in future papers. 3C 289 also has a likely over-density of objects in its immediate vicinity.

\section{Modelling the Quasars with the Radio Galaxies}

In order to understand how the radio galaxy properties compare with those of the quasars, we must address how the radio galaxies we have observed would appear if they were superposed on quasar nuclei. Though we can estimate how much of the radio galaxy would certainly have been lost in a nuclear subtraction by simply excluding the central region as we have done in the magnitude and moment analyses discussed above, this does not include the considerable noise that may be introduced in the subtraction process. If the PSF is observed (as are the ones we use here) rather than modelled, then subtraction adds sky noise (partially flat-fielding residuals, partially Poisson) and Poisson noise from the overlying bright nucleus. In addition, the details of any observation (jitter, positioning on the chip) will introduce PSF variations, so another potential source of noise is the PSF subtraction residual.

To model as closely as possible the quasars as we observed them, we would like to have a PSF independent of the stellar PSFs to simulate the quasar nuclei. If we use the same stellar PSFs to subtract that we use to model the nuclei (with independent noise added, 
of course), we may succeed in simulating the effect of the Poisson noise, but will not be able to address the deviations caused by PSF mismatches. The modelled Tiny Tim PSFs show large residuals when subtracted from observed PSFs, and they are not an adequate representation of the actual PSFs in our fields. We have therefore tried to obtain an average PSF from the quasar nuclei themselves.

We take the host galaxies and other residual structure after the subtraction of the PSFs for each of our quasars and interpolate over the inner region that is dominated by noise and PSF mismatch residuals. We slightly smooth these images and mask off regions that are essentially just sky. We then subtract these images from the corresponding original quasar images and combine these host-galaxy-subtracted PSFs with gcombine and the "rsigclip" clipping algorithm, giving us a fairly clean PSF that is largely independent of our stellar PSFs.

We scale this PSF to simulate each of our observed quasar profiles, and we then superpose each of these 5 PSFs on each of the 5 radio galaxies, adding in the appropriate noise. More details of this modelling process are given by Ridgway (1995).

We then run the same centering, scaling, and monotonic subtraction procedures described in $\S 4.2$ on these 25 images. These models are on the conservative side; some of the radio galaxy images had higher sky noise than the final quasar image we were attempting to simulate. But they give a good impression of some of the effects of the Poisson noise and PSF mismatches. (For the opposite, optimistic limit, we have in essence simulated a near-perfect "noiseless" subtraction by simply removing the central region, as discussed in A.2).

We display a montage of the subtracted images in Fig. 22. The first row shows the observed radio galaxies; they are oriented as observed on the WFC CCD. The second row shows the unsubtracted models of $3 \mathrm{C} 245$, consisting of an average PSF (scaled to the magnitude of the actual 3C 245 nucleus) with each of the observed radio galaxies added. $3 \mathrm{C} 245$ is the brightest of our quasars, and this row shows therefore the case for which it is most difficult to recover the extensions. The next 5 rows show subtracted versions of the models of each of the 5 quasars, constructed in a similar fashion. In each case, we subtract the average stellar PSF appropriate for the filter in which the quasar was actually observed, to give an idea of the range of possible residuals. The distance between tickmarks represent $1^{\prime \prime}$.

Visual inspection of the models reveals some interesting points. As expected, it is easier to recover host galaxy structure from fainter QSOs like 3C 2 and $3 \mathrm{C} 212$. 3C 2 also happens to have been fairly deeply observed, and therefore to have lower sky noise. We 
also see an extra feature to the upper right in the 3C 245 and 3 C 336 simulations; this may correspond to a poor subtraction of the diffraction spike in this corner, but it is also visible in the difference between the average stellar F622W PSF and F675W PSF. We get an idea from these models out to what radius PSF residuals are likely to dominate our recovered data: we find that the morphologies and fluxes are generally little affected outside our adopted radius of 0.45 .

This suite of models also allows us to make a better comparison of the alignments in the two samples. We would be able to detect closely aligned structure under most of the model nuclei in only the two most aligned radio galaxies in our sample, 3C 280 and 3C 237.

In our sample of actual observed quasars, if we exclude the contributions of optical synchrotron emission, we see similar aligned continuum structures in 3C 196 and in 3C 336 (if we disregard the nearby components spectroscopically determined to be unassociated with the 3 C 336 host galaxy). We thus find that the incidence of "standard" aligned material is certainly consistent between the two samples.

\section{Discussion}

\subsection{Alignment Effect}

A clear prediction of unified models for quasars and FR II radio galaxies is that the quasar hosts should show an alignment effect, but with reduced amplitude and with larger misalignment because of projection.

Though the alignment effect is certainly a statistically significant property of large samples of high-radio-luminosity, high-redshift radio galaxies, individual objects seem to exhibit different kinds of aligned morphologies and these structures probably differ in origin. It is therefore often necesssary to consider the observational evidence relevant to each individual object.

The morphologies observed by HST in the UV for many of the $z \sim 1$ radio galaxies (Longair et al. 1995, Best et al. 1996, Dickinson et al. 1995) argue strongly for the interaction of the jet with the ambient medium being the primary cause of the structures observed. The curved, linear, discrete structure of $3 \mathrm{C} 324$ which tracks the jet direction leaves little room for doubt. Still, however, it is debatable exactly what material is involved, what the jet interaction caused, and what the actual emission mechanism for the aligned UV light is. Jet-induced star formation as the source of the aligned UV lumps in most of these smaller scale radio sources is basically consistent with the observed HST morphologies; 
however, some of these same sources (such as 3C 324; Cimatti et al. 1996) show evidence for a significant level of polarization. This polarization percentage is consistent with the bulk of the UV light coming from dust scattering, with dilution coming from an evolved stellar population showing a significant $4000 \AA$ break.

In addition, the thermal nebular continuum has been shown to be a major contributor to the aligned component of 3C 368 (Dickson et al. 1995; Stockton et al. 1996), which we now know to be unpolarized (Dey et al. 1997). This means that even among the "small-scale, very aligned" examples, we have one in which scattered light is probably the dominant contributor of UV light (3C 324) and one in which nebular continuum is dominant (3C 368).

In our sample, we see evidence for a number of the mechanisms that are considered possible contributors to the alignment effect. Most obviously, we see a number of quasars (but no radio galaxies) in which we almost certainly have beamed optical synchrotron emission: $3 \mathrm{C} 2,3 \mathrm{C} 212$, and $3 \mathrm{C} 245$. In the quasars $3 \mathrm{C} 196$ and $3 \mathrm{C} 336$, and in the radio galaxies 3C 237 and 3C 280, we see examples of alignment that apparently cannot be attributed to synchrotron emission. In the three of these objects for which we have the necessary observations, we detect aligned [O II] emission. We see evidence of illumination (scattering or photoionization) effects in the edge brightening observed in the arc in $3 \mathrm{C} 280$, and possibly in the very blue objects within the "scattering cone" of 3C 196 . The arc and peak structure in $3 \mathrm{C} 280$ has a strong contribution from thermal continuum emission from ionized gas. The only major proposed alignment mechanism for which we have no direct evidence is jet-induced star formation.

In summary, in our sample, we find evidence for most of the major mechanisms for alignment, and we find neither support nor counter-evidence for jet-induced star formation. From these purely morphological studies we cannot, however, pin down certain origins for most of the aligned components. Follow-up studies of key objects and components, such as the NW component in $3 \mathrm{C} 212$, may prove vital for understanding the kind of multi-component morphology observed in many radio galaxies.

\subsection{Unification Hypothesis}

Past comparisons of low-redshift radio galaxy and quasar host properties have been inconclusive and contradictory, primarily because of large uncertainties introduced by the PSF subtraction, and lack of complete samples. An example of such a contradiction is in the studies of Dunlop et al. (1993), who find that $z \sim 0.3$ radio galaxy and quasar host 
magnitudes are the same, and of Smith \& Heckman (1989) who find that host magnitudes in similar samples differ significantly. Our magnitude and alignment effect results are basically consistent with a standard FR II unification model but do not provide a strong test of it because of the limited size of our radio galaxy and quasar samples. Indeed, we expect an intrinsic dispersion in properties such as opening angle, and this variation could be enough to hide a statistical difference in observed properties between radio galaxy and quasar samples.

We also detect faint unresolved cores in all of our HST radio galaxy images. The brightnesses of these cores seem to roughly correlate with the radio core brightnesses (e.g., $3 \mathrm{C} 217$ has the faintest optical core and is undetected in the radio). These optical cores may be the obscured active nuclei.

\subsection{Optical Synchrotron Emission}

In at least three of our sources, we have found detailed correspondences between optical and radio structure that can plausibly be interpreted as optical synchrotron emission. In one case, $3 \mathrm{C} 2$, the emission comes from the north-eastern lobe at a projected separation of $\sim 6 \mathrm{kpc}$; in the others, we have evidence for an optical counterparts to radio jets on the northeastern side of the core of $3 \mathrm{C} 212$ and the western side of the core of $3 \mathrm{C} 245$. In $3 \mathrm{C} 212$, the physical separation from the farthest "jet knot" to the nucleus is $\sim 20 \mathrm{kpc}$; in $3 \mathrm{C} 245$, the jet extends to a length of $\sim 10 \mathrm{kpc}$.

Likely examples of optical synchrotron emission have been detected previously in about a dozen other extragalactic objects. (Crane et al. 1993 and references therein; Meisenheimer et al. 1989 and references therein). Their radio/optical spectral properties have many similarities, and they generally fit a common pattern often found for astrophysically observed synchrotron emission: that of a power law with $\alpha \sim 0.5-0.8$ (where $S_{\nu} \propto \nu^{-\alpha}$ ), with a steepening of the power law spectrum at higher frequencies above a certain cutoff frequency $\nu_{c}$.

An alternative mechanism for generating optical emission from a distribution of relativistic electrons is the inverse Compton effect, which can upconvert low-frequency photons to the optical region via scattering from the relativistic electrons (Daly $1992 b$ and references therein). This process is normally important at low redshifts only in the context of contributing to the cooling of the lower-energy electrons in compact sources with very high radiation densities. However, since the energy density of the microwave background increases as $(1+z)^{4}$, at a sufficiently high redshift this energy density will be comparable 
to that of the magnetic field in typical radio lobes or jets, and the inverse Compton process may dominate the observed emission (Daly 1992b).

We consider the case of the north-eastern lobe of $3 \mathrm{C} 2$, for which resolved radio data at multiple frequencies is available (Saikia et al. 1987). Estimating the magnetic field from the minimum-energy condition, using the usual (but not necessarily correct) assumptions of unity filling factor, an equal energy division between electrons and protons, uniform magnetic field, and source depth along the line of sight equal to the observed source width (e.g., Miley 1980), we obtain $B_{\perp} \approx 10^{-4}$ Gauss. The NE lobe of $3 \mathrm{C} 2$ dominates the total flux from the source at all radio frequencies for which it was resolved, and the total spectral index is nearly constant over a range of 3 decades in frequency. This constancy in spectral index allows us to use Eqn $8 b$ of Daly (1992b) to estimate the flux density due to inverse Compton scattering in the NE lobe. We obtain $f_{I C}=2.2 \times 10^{-21} \mathrm{erg} \mathrm{cm}^{-2} \mathrm{~s}^{-1} \AA^{-1}$. Our observed value of $\sim 10^{-18} \mathrm{erg} \mathrm{cm}^{-2} \mathrm{~s}^{-1} \AA^{-1}$ indicates that the optical emission is strongly dominated by synchrotron radiation rather than inverse Compton scattering of microwave background photons.

Further support for this result comes from the break we see between the spectral index in the radio and optical regions (Fig. 14). The inverse Compton process should produce optical/IR radiation with a spectral index like that of the synchrotron radiation from the (low-energy) relativistic electrons responsible for the upscattering, i.e., typically that at $\sim 1$ $\mathrm{MHz}$ (e.g., Daly 1992b). In general, radio spectral indices at such frequencies are unlikely to be steeper than those at centimeter to meter wavelengths, so our steeper optical/IR spectral index is evidence for optical synchrotron emission, where the synchrotron spectrum has a high-frequency break normally resulting from the aging of the electron energy distribution due to synchrotron losses. This effect is shown clearly in the Fermi acceleration models of Meisenheimer et al. (1989).

De Koff et al. (1997) note that, for their $z<0.23 \mathrm{C}$ galaxy sample, the angular radio sizes of those galaxies showing optical synchrotron emission are less than those in which no such detection is made. They suggest that this is due to beaming effects; i.e., that the radio galaxies with optical synchrotron detections have jet axes that are preferentially aligned closer to our line of sight. This interpretation is consistent with our finding evidence for optical synchrotron emission in most of our quasars, but in none of our radio galaxies. 


\section{Summary}

With our HST imaging going nearly as deeply as our best previous ground-based imaging (even on low-surface-brightness features), HST's improved resolution over the ground-based observations makes a significant difference in our interpretation of the extensions observed around the quasars. Often structure that seems nebulous and galaxy-like with ground-based seeing is revealed to consist of discrete components that may have more to do with the AGN than with stellar populations. An example of this is the NE lobe of $3 \mathrm{C} 2$; without the high-resolution image it would likely be considered a portion of the host galaxy, and perhaps evidence for a second nucleus, rather than identified as optical synchrotron emission. For the radio galaxies, on the other hand, this improvement in resolution in some cases may give entirely new information, as for $3 \mathrm{C} 280$, while in others it mainly confirms what could be surmised about the radio galaxy structure from lower-resolution data (e.g., 3C 217 or $3 \mathrm{C} 289$ ).

Our samples are small, but some results are clearcut:

We find evidence for continuum structure around all of our quasars in the high resolution WFPC2 data, although generally much of the extension does not resemble a "normal" galaxy host. 3C 2 seems, however, to have a bright elliptical galaxy-like nebulosity. We resolve structure around 4 of the 5 quasars in the near-infrared images.

We observe a high incidence of morphological oddities, all sometimes ascribed to interactions: a high incidence of nearby companion galaxies, lumpy morphology, and asymmetric emission-line gas. In addition, we see an arc in 3C 280 which bears morphological resemblance to a tidal tail.

We see morphological and color evidence for illumination effects from the active nucleus, i.e., scattered quasar light or photoionization. In 3C 196 and 3C 280, we see very blue and/or edge-brightened structures that lie within the probable quasar opening angle.

We see evidence for aligned emission in all of our quasars. In $3 \mathrm{C} 212$, we see an object that lies beyond the radio lobe but looks morphologically quite similar to a radio hotspot and tail; this object is bright in the infrared and has a steep spectral gradient across the tail. If this is truly a result of the radio jet, it is a unique object that may prove vital to understanding the relationship between the UV continuum alignment effect and the radio source. We have detected optical counterparts to radio jets in the quasars 3C 212 and $3 \mathrm{C} 245$, and an optical counterpart to a radio lobe in $3 \mathrm{C} 2$. All of these structures have such a detailed, high-resolution, point-to-point correspondence with the radio structures that they are very likely the result of optical synchrotron radiation, and the spectral indices in $3 \mathrm{C} 2$ are consistent with this interpretation. In $3 \mathrm{C} 196$ and $3 \mathrm{C} 336$, we see aligned structure 
that does not appear to correlate directly with radio emission and that seems to be similar to the aligned UV components seen in radio galaxies.

The colors of the extended components around the quasars are in all cases similar to or redder than that of the quasar nucleus. In some cases at least, nearby companions or overall nebulosity have colors consistent with a stellar population of 4 Gyr or more. Thus, the emission we see around the quasars and in the radio galaxies can probably be ascribed to the following mechanisms:

1) optical synchrotron in three quasars: $3 \mathrm{C} 2,3 \mathrm{C} 212$, and $3 \mathrm{C} 245$

2) stellar host galaxies, particularly seen in the near-infrared

3) scattered quasar light or thermal continuum in some components and companions

4) true nearby companion galaxies

5) "standard" UV aligned emission, for which the origin is uncertain.

We find that the optical and infrared flux densities of the extended material are consistent in the radio galaxy and quasar subsamples within the dispersion of our small sample, and that this result is not dependent on the contribution of the beamed optical synchrotron components detected in the quasars and not in the radio galaxies. The mean and dispersion of the infrared magnitudes of the quasar extensions (after correction for the missing flux from the obscured central region) are consistent with the $K-z$ relation found for radio galaxies. We find the quasar and radio galaxy hosts have $K^{\prime}$ luminosities of $L^{*}$ or slightly less.

Our elliptical profile fits to the $K^{\prime}$ images of some of the radio galaxies indicate that their surface-brightness distributions are well fit by an $\mathrm{r}^{\frac{1}{4}}$ law. These profiles, and the red colors we have found for these components, suggest that at rest-frame $1 \mu \mathrm{m}$ these radio galaxies are often dominated by an old (3-4 Gyr) stellar population.

From the correspondence between the total magnitudes in the radio galaxies and quasars, and the detection of aligned components in quasars, we conclude that this study provides general support for the unification hypothesis of radio galaxies and quasars. These issues are confused by the wide range of morphological types found among the aligned components and by the small size of our sample. Nevertheless, 4 of the 5 quasars show some extended material that can plausibly be attributed to stars, and 3C 196 and 3C 336 have aligned components that are unlikely to be due to optical synchrotron radiation, and appear qualitatively similar to those often seen in high-redshift radio galaxies.

We are currently obtaining WFPC2 imaging of an additional $53 \mathrm{CR}$ quasars from our 
larger complete sample $(\S 2)$. These observations will double the number of quasars for which we have WFPC2 imaging, and this increase in sample size will allow us to reduce the uncertainties caused by the small number of objects in the current work. With additional ground-based imaging and deep spectroscopy of individual fields, we hope to make further progress in understanding the properties of quasars at $z \sim 1$ and their relationship with radio galaxies.

We thank Ken Chambers, Esther Hu, Mark Lacy, and Neil Trentham for reviewing and commenting on various versions of the paper. We are very grateful to Mark Lacy and Katherine Blundell for guidance in the reduction our VLA data. We also thank Neil, Mark, Katherine, Steve Rawlings, Joss Bland-Hawthorne and Ruth Daly for the time they have spent and interest they have shown in discussing the alignment effect in general and 3C 212 in particular with us. Comments from the referee, Patrick McCarthy, helped us improve both the content and the presentation of this paper. We thank Patrick Leahy for a critical evaluation of the best radio maps of our sources in the literature, and we thank Sylvia Baggett at STScI for her help in recalibrating the HST data. Jonathan Higa wrote the original versions of the IRAF scripts we used for IR image reduction and PSF subtraction. The authors were visiting astronomers at the NASA Infrared Telescope Facility, operated by the University of Hawaii under contract with NASA. Support for this work was provided by NASA through grant number GO-05401.01-93A from the Space Telescope Science Institute, which is operated by AURA, Inc., under NASA contract NAS 5-26555. Some of the ground-based observations were supported by NSF grant AST 92-21909, and SER was supported by a PPARC Postdoctoral Fellowship while finishing this work.

\section{A. Magnitude and Moment Analysis of the Images}

\section{A.1. Magnitudes}

We discussed in 4.3 how we created isophotal masks from the images (with flux densities normalized to $z=1$ ) in order to allow us to compare the imaging results for our objects less biassed by the details of our observations. Here we will give more details and discuss the differences in the way we treated the ground-based data from the HST images.

For the WFPC2 images, we first define a single set of isophotal levels; these are chosen based on our knowledge of the average normalized $1 \sigma$ sky in our observed frames, given in Table 2. For each image, multiplied by the $z=1$ correction factor, we create masks at these isophotal levels. We then smooth the mask contours slightly by convolving with a Gaussian 
with a sigma of 1 rebinned pixel $\left(0^{\prime \prime} 05\right)$ and excluding any pixels whose "smoothed" values are less than 0.5 . This smoothing tends to eliminate any isolated sky pixels which might have been picked up in the mask and gives continuous contours; it therefore allows us to consider magnitudes to slightly lower flux limits than would have been otherwise possible. Otherwise, choosing a flux level at which all images will not have noise-dominated profiles would exclude much of the interesting features in the quasars. (As it is, we still will not include in the objective magnitude comparison some low-level nebulosity which falls below our best "common" isophotal level).

We then calculate the flux densities in the unsmoothed image after multiplication by these smoothed masks (in a series of apertures). We find this smoothing process works well on radio galaxies, but tends to lose flux from linear features such as those seen in 3C 212 and $3 \mathrm{C} 245$. For this reason we reduced the smoothing as much as possible and checked the smoothed lowest isophotal masks visually to see how much of the structure of interest we are preserving. From this visual inspection, we also select the lowest isophotal level at which all of our normalized image masks have little or no sky contribution. We take the lowest common level to be $4.56 \times 10^{-19} \mathrm{ergs} \mathrm{cm}^{-2} \mathrm{~s}^{-1} \AA^{-1} \operatorname{arcsec}^{-2}$ ), about 2.5 times the average normalized sky $\sigma$. For the $K^{\prime}$ data, we follow a very similar procedure, except that we do not need to smooth the masks, prior to applying them to the images. Our adopted "common" isophotal level for the $K^{\prime}$ data is $1.4 \times 10^{-19} \mathrm{ergs} \mathrm{cm}^{-2} \mathrm{~s}^{-1} \AA^{-1} \operatorname{arcsec}^{-2}$.

This magnitude calculation procedure is straightforward for the radio galaxies; for the quasars we must deal with the interior region of the subtracted image which is dominated by residuals from the profile subtraction. As a first pass, we mask out the profile subtraction region in the quasars to exclude them from our moment and magnitude calculations; a circular region with radius 0.45 is generally necessary to remove the region dominated by PSF subtraction rediduals in the HST images. We also mask out an identical region in the radio galaxies to make an accurate comparison, and to understand how this subtraction process affects the final magnitudes we calculate. We have also PSF-subtracted the radio galaxies, and verified that outside of this masked-off central region, the wings of the PSF do not significantly contribute to the radio galaxy annular fluxes. For the near-infrared radio galaxy images, the extended flux is mostly quite dominant, and the best PSF-subtraction is just an estimate of where the galaxy profile obviously is no longer monotonic. We follow the entire masking and magnitude- and moment-determination procedure for both subtraction limits for those quasars in which the monotonic limit differed significantly from the $\chi^{2}$ minimum fit. These $\chi^{2}$ minimum subtractions give us a probable lower-limit to the flux we observe around the quasars and an idea of whether changing our PSF-subtraction technique would affect our results significantly. We then carry out a similar set of calculations, where, instead of masking out the central region, we interpolate across it with a low-order 
2-dimensional fit.

We include companions within $3^{\prime \prime}$ for our objective comparison; however, in some cases (like those of $3 \mathrm{C} 280$ and $3 \mathrm{C} 289$ ) we found it instructive to consider the central component alone as well. We also exclude from our moment and magnitude analyses known foreground objects, such as the probable $z=0.437$ absorbing spiral galaxy in front of 3C 196 (BB), the star in the 3C 175.1 field, the probable foreground galaxy in the 3C 280 field, (for most purposes) the $z=1.013$ elliptical galaxy companion to 3C 245 (LeFèvre \& Hammer 1992) and the foreground galaxy $d$ in the 3C 336 field.

\section{A.2. Position Angle Determination}

To quantify the alignments of the objects in our sample, we adopt a technique similar to that of Dunlop \& Peacock (1993), with some exceptions. First, we explicitly mask out any known foreground or companion objects, particularly those for which likely redshifts exist. We then make our moment calculations in the isophotal masks we have generated, with a common range of apertures (from $2^{\prime \prime}$ up to $10^{\prime \prime}$ in diameter) that should span the probable range of physical scales of interest for all of the objects.

To find the principal axes of the flux distribution, we calculate the first and second flux-weighted moments. Where $\mu_{i}$ is the sky-subtracted intensity of the $i$ 'th pixel at position $\left(x_{i}, y_{i}\right)$, we calculate $\sum_{i}\left(\mu_{i}\right)$ and the first moments $\sum_{i}\left(x_{i} \mu_{i}\right) / \sum_{i}\left(\mu_{i}\right)$ and $\sum\left(y_{i} \mu_{i}\right) / \sum\left(\mu_{i}\right)$. We then calculate the second moments around the center that most closely matches a probable AGN location. For our quasars, this is simply the quasar center; for our radio galaxies, we detect unresolved optical contributions in all of the HST images which match the best infrared centers. We therefore use in all cases the WFPC2-determined "nuclear" center. (In all of our objects the optical and near-IR cores match well, thus we use the higher resolution HST data to determine the best center. Such small shifts in centering affect the moments little). We derive the second moments $I_{x x}=\sum_{i}\left(x_{i}^{2} \mu_{i}\right) / \sum_{i}\left(\mu_{i}\right), I_{y y}=\sum_{i}\left(y_{i}^{2} \mu_{i}\right) / \sum_{i}\left(\mu_{i}\right)$, $I_{x y}=\sum_{i}\left(x_{i} y_{i} \mu_{i}\right) / \sum_{i}\left(\mu_{i}\right)$, where $\left(x_{i}, y_{i}\right)$ is the position of the $i$ 'th pixel relative to the best determined center. We use these to determine the position angle $\theta$ and the ratio of the minor to major axis $a / b$ of the equivalent ellipse.

\section{REFERENCES}

Abraham, R.G., Crawford, C.S., \& McHardy, I.M. 1992, ApJ, 401, 474

Akujor, C.E., Spencer, R.E., Zhang, F.J., Davis, R.J., Browne, I.W.A., \& Fanti, C. 1991, 
MNRAS, 250, 214

Antonucci, R. 1993 ARA\&A, 31, 473

Antonucci, R., \& Barvainis, R. 1990, ApJ, 363, L17

Antonucci, R., Hurt, T., \& Kinney, A. 1994, Nature, 371, 313

Arextaga, I., Boyle, B.J., \& Terlevich, R.J. 1995, MNRAS, 275, L27

Bahcall, J.N., Kirhakos, S., \& Schneider, D.P. 1994, ApJ, 435, L11

Bahcall, J.N., Kirhakos, S., \& Schneider, D.P. 1995a, ApJ, 447, L1

Bahcall, J.N., Kirhakos, S., \& Schneider, D.P. 1995b, ApJ, 450, 486

Bahcall, J.N., Kirhakos, S., \& Schneider, D.P. 1996, ApJ, 457, 557

Bahcall, J.N., Kirhakos, S., Saxe, D.H., \& Schneider, D.P. 1997, ApJ, 479, 642

Barthel, P.D., Miley, G.K. 1988, Nature, 333, 319

Barthel, P.D. 1989, ApJ, 336, 606

Baum, S.A., \& Heckman, T. 1989, ApJ, 336, 681

Begelman, M.C., \& Cioffi, D.F. 1989, ApJ, 345, L21

Bennett, A.S. 1962, MmRAS, 68, 163

Best, P.N., Longair, M.S., \& Röttgering, H.J.A. 1996, MNRAS, 280, L9

Biretta, J.A., Stern, C.P., \& Harris, D.E. 1991, AJ, 101, 1632

Blundell, K.M., \& Alexander, P. 1994, MNRAS, 267, 241

Boisse, P., \& Boulade, O. 1990, A\&A, 236, 291 (BB)

Bremer, M.N. 1997, MNRAS, 284, 126

Bremer, M.N., Crawford, C.S., Fabian, A.C., \& Johnson, R. M. 1992, MNRAS, 254, 614

Bridle, A.H., Hough, D.H., Lonsdale, C.J., Burns, J.O., \& Laing, R.A. 1994, AJ, 108, 766

Brodie, J.P., Bowyer, S. \& McCarthy, P. 1985, ApJ, 293, L59

Bruzual, G \& Charlot, S. 1993, ApJ, 405, 538

Burstein, D., \& Heiles, C. 1984, ApJS, 54, 33

Carilli, C.L., Perley, R.A., \& Dreher, J.H. 1988, ApJ, 334, L73

Casali, M. \& Hawarden, T. 1992, JCMT-UKIRT Newsletter, Aug. 1992, 33

Chambers, K.C., Miley, G.K., \& Joyce, R.R. 1988, ApJ, 329, L75

Chambers, K.C., Miley, G., \& van Breugel, W. 1987, Nature, 329, 609 
Chambers, K.C., \& McCarthy, P.J. 1990, ApJ, 354, L9

Clark, N.E., Tadhunter, C.N., Axon, D.J., \& Robinson, A. 1997, in the on-line proceedings of the Sheffield workshop on "Jet-cloud interactions in active galaxies", http://www.shef.ac.uk/ phys/research/astro/conf/

Cimatti, A., di Serego Alighieri, S., Fosbury, R.A.E., Salvati, M., \& Taylor, D. 1993, MNRAS, 264, 421

Cimatti, A., Dey, A., van Breugel, W., Antonucci, R., \& Spinrad, H. 1996, ApJ, 465, 145

Cohen, R.D., Beaver, E.A., Diplas, A., Junkkarinen, V.T., \& Lyons, R.W. 1996, ApJ, 456, 132.

Coleman, G.D., Wu, C.C., \& Weedman, D.W. 1980, ApJS, 43, 393

Crane, P. et al. 1993, ApJ, 402, L37

Crawford, C.S., \& Fabian, A.C. 1995, MNRAS, 273, 827

Daly, R.A. 1990, ApJ, 355, 416

Daly, R.A. 1992a, ApJ, 386, L9

Daly, R.A. 1992b, ApJ, 399, 426

de Koff, S., Baum, S.A., Sparks, W.B., Biretta, J., Golombek, D., Macchetto, F., McCarthy, P., \& Miley, G.K. 1997, ApJS, in press

de Young, D.S. 1989, ApJ, 342, L59

Dey, A. \& van Breugel, W.J.M. 1994, AJ, 107, 1977.

Dey, A., Cimatti, A., van Breugel, W., Antonucci, R., \& Spinrad, H. 1996, ApJ, 465, 157

Dey, A, van Breugel, W., Graham, J., Spinrad, H., Cimatti, A., Hurt, T., \& Antonucci, R. 1997 , in preparation

Dickson, R., Tadhunter, C., Shaw, M., Clark, N., \& Morganti, R. 1995, MNRAS, 273, L29

Dickinson, M., Dey, A., \& Spinrad, H. 1995, in Galaxies in the Young Universe, edited by H. Hippelein (Springer-Verlag, Heidelberg), p. 164

di Serego Alighieri, S., Cimatti, A., \& Fosbury, R.A.E. 1994, ApJ, 431, 123

Disney, M.J., Boyce, P.J., Blades, J.C., Boksenberg, A., Crane, P., Deharveng, J.M., Macchetto, F., Mackay, C.D., Sparks, W.B., \& Phillipps, S. 1995, Nature, 376, 150

Djorgovski, S., Spinrad, H., Pedelty, J., Rudnick, L., \& Stockton, A. 1987, AJ, 93, 1307

Dunlop, J.S., \& Peacock, J.A. 1993, MNRAS, 263, 936

Dunlop, J.S., Peacock, J.A., Spinrad, H., Dey, A., Jiminez, R., Stern, D. \& Windhorst, R. 1996, Nature, 381, 581 
Dunlop, J.S., Taylor, G.L., Hughes, D.H., \& Robson, E.I. 1993, MNRAS, 264, 455

Eales, S.A, Rawlings, S., Law-Green, D. Cotter, G., \& Lacy. M. 1997, MNRAS, in press

Elias, J.H., Frogel, J.A., Matthews, K. \& Neugebauer, G. 1982, AJ, 87, 1029

Fabian, A.C., 1989, MNRAS, 238, 41P

Fanti, R., Fanti, C, Schilizzi, R.T., Spencer, R.E., Nan, R.D., Parma, P., van Breugel, W.J.M., \& Venturi, T. 1990, A\&A, 231, 333

Garrington, S.T., Leahy, J.P., Conway, R.G., \& Laing, R.A. 1988, Nature, 331, 147

Garrington, S.T. \& Conway, R.G. 1991, MNRAS, 250, 198

Ghisellini, G., Padovani, P., Celotti, A., \& Maraschi, L. 1993, ApJ, 407, 65

Hansen, L, Nørgaard-Nielsen, H.U, \& Jørgensen, H.E. 1987, A\&AS, 71, 465

Heckman, T.M. 1990, in IAU Colloquium 124, Paired and Interacting Galaxies, edited by J.W. Sulentic, W.C. Keel, and C. Telesco (NASA CP-3098), p. 359

Heckman, T.M., Lehnert, M.D., van Breugel, W., \& Miley, G.K. 1991, ApJ, 370, 78

Heckman, T.M., Chambers, K.C., \& Postman, M. 1992, ApJ, 391, 39

Herbig, T., \& Readhead, A.C.S. 1992, ApJS, 81, 83

Hill, G.J., Goodrich, R.W., \& DePoy, D.L. 1996, ApJ, 462, 163

Hintzen, P., Romanishin, W., \& Valdes, F. 1991, ApJ, 366, 7

Holtzman, J.A., Burrows, C.J., Casertano, S., Hester. J.J., Trauger, J.T., Watson, A.M., \& Worthey, G. 1995, PASP, 107, 1065

Hutchings, J.B., Holtzman, J., Sparks, W.B., Morris, S.C., Hanisch, R.J., \& Mo, J. 1994a, ApJ, 429, L1

Hutchings, J.B., Neff, S.G., Weadock, J., Roberts. L, Ryneveld, S., \& Gower, A.C. 1994b, AJ, 107, 994

Jedrzejewski, R.I. 1987, MNRAS, 226, 747

Kühr, H., Witzel, A., Pauliny-Toth, I.I.K., \& Nauber, U. 1981, A\&AS, 45, 367

Lacy, M. 1997, in the on-line proceedings of the Sheffield workshop on "Jet-cloud interactions in active galaxies", http://www.shef.ac.uk/ phys/research/astro/conf/

Lacy, M., Rawlings, S., Eales, S., \& Dunlop, J. S. 1995, MNRAS, 273, 821.

Laing, R.A., Riley, J.M., \& Longair, M.S. 1983, MNRAS, 204, 151

Laing, R. 1988, Nature, 331, 149 
Laing, R. 1989, in Hotspots in Extragalactic Radio Sources, (Springer-Verlag, Heidelberg), p. 27

Le Brun, V., Bergeron, J., Boissé, P., \& Deharveng, J.M. 1997, A\&A, in press

LeFèvre, O., \& Hammer, F. 1992, A\&A, 254, L29

Lehnert, M.D., Heckman, T.M., Chambers, K.C., \& Miley, G.K. 1992, ApJ, 393, 68

Lilly, S.J. 1989, ApJ, 340, 77

Liu, R., \& Pooley, G.G. 1991, MNRAS, 253, 669

Liu, R., Pooley, G.G., \& Riley, J.M. 1992, MNRAS, 257, 545

Longair, M.S., Best, P.N., \& Rottgering, H.J.A. 1995, MNRAS, 275, L47

Lowenthal, J.D., Heckman, T.M., Lehnert, M.D., \& Elias, J.H., 1995, 439, 588

Massey, P., Strobel, K., Barnes, J.V., \& Anderson, E. 1988, ApJ, 328, 315

Massey, P. \& Gronwall, C. 1990, ApJ, 358, 344

McCarthy, P.J. 1993, ARA\&A, 31, 639

McCarthy, P.J., Spinrad, H., van Breugel, W. 1995, ApJS, 99, 27

McCarthy, P.J., \& van Breugel, W.J.M. 1989, in Epoch of Galaxy Formation, edited by C.S. Frenk, R.S. Ellis, T. Shanks, A.F. Heavens, and J.A. Peacock (Kluwer, Dordrech), p. 57

McCarthy, P.J., van Breugel, W., Spinrad, H., \& Djorgovski, S. 1987, ApJ, 321, L29

McLeod, K.K., \& Rieke, G.H. 1994a, ApJ, 420, 58

McLeod, K.K., \& Rieke, G.H. 1994b, ApJ, 431, 137

McLeod, K.K., \& Rieke, G.H. 1995, ApJ, 454, L77

Meisenheimer, K., Roser, H.-J., Hiltner, P.R., Yates, M.G., Longair, M.S., Chini, R., \& Perley, R.A. 1989, A\&A, 219, 63

Mihos, J.C. 1995, ApJ, 438, L75

Miley, G.K. 1980, ARA\&A, 18, 165

Miley, G.K., Chambers, K.C., van Breugel, W.J.M., \& Macchetto, F. 1992, ApJ, 401, L69

Mobasher, B., Sharples, R.M., \& Ellis, R.S. 1993, MNRAS, 263, 560

Neff, S.G., Roberts, L., \& Hutchings, J.B. 1995, ApJS, 99, 349

Pedelty, J.A., Rudnick, L., McCarthy, P.J., \& Spinrad, H. 1989, AJ, 97, 647

Rees, M.J. 1989, MNRAS, 239, 1P 
Reid, A., Shone, D.L., Akujor, C.E., Browne, I.W.A., Murphy, D.W., Pedelty, J, Rudnick, L. \& Walsh, D. 1995, A\&AS, 110, 213

Ridgway, S.E. 1995, Ph.D. thesis, University of Hawaii.

Ridgway, S.E., \& Stockton, A. 1997a, in preparation.

Ridgway, S.E., \& Stockton, A. 1997b, in preparation.

Rigler, M.A., Lilly, S.J., Stockton, A., Hammer, F., \& LeFèvre, O. 1992, ApJ, 385, 61

Saikia, D.J., \& Kulkarni, V.K. 1995, MNRAS, 270, 897

Saikia, D.J., Salter, C.J., \& Muxlow, T.W.B. 1987, MNRAS, 224, 911

Scheuer, P. 1987, in Superluminal Radio Sources, edited by J. Zensus and T. Pearson (Cambridge Univ. Press, Cambridge), p. 104

Smith, E.P., \& Heckman, T.M. 1989, ApJ, 341, 658 ApJ, 361, L41

Spinrad, H., Djorgovski, S., Marr, J. \& Aguilar, L. 1985, PASP, 97, 932

Steidel, C.C. \& Dickinson, M. 1992, ApJ, 394, 81

Steidel, C.C., Dickinson, M., Meyer, D.M., Adelberger, K.L., \& Sembach, K.R. 1997, ApJ, 480 , in press

Stockton, A. 1990. in Dynamics and Interactions of Galaxies, edited by R. Wielen (Springer-Verlag, Heidelberg), p. 440

Stockton, A., Ridgway, S.E., \& Lilly, S.J. 1994, AJ, 108, 414

Stockton, A., Kellogg, M., \& Ridgway, S.E. 1995, ApJ, 443, L69

Stockton, A., Ridgway, S.E., \& Kellogg, M. 1996, AJ, 112, 902

Stockton, A., \& Ridgway, S.E. 1997, in preparation

Tadhunter, C., Shaw, M., Clark, N., \& Morganti, R. 1994, A\&A, 288, L21

Urry, C.M. \& Padovani, P. 1995, PASP, 107, 803

van Breugel, W., Filippenko, A.V., Heckman, T., \& Miley, G. 1985, ApJ, 293, 83

van Breugel, W.J.M., Fanti, C., Fanti, R., Stanghellini, C., Schilizzi, R.T., \& Spenser, R.E. 1992, A\&A, 256, 56

Wainscoat, R.J., \& Cowie, L.L. 1992, AJ, 103, 332

West, M.J. 1994, MNRAS, 268, 79

Williams, A.G. 1991, in Beams and Jets in Astrophysics, edited by P.A. Hughes (Cambridge Univ. Press, Cambridge), p. 342

Williams, A.G., \& Gull, S.F. 1985, Nature, 313, 34 
Worrall, D.M., Lawrence, C.R., Pearson, T.J., \& Readhead, A.C.S. 1994, ApJ, 420, L17 
Fig. 1.- The results of subtracting our observed stellar PSFs from each other with the centering and scaling technique described in text. The images are to the same scale as the magnified panels in Figs 3-12; the distance between tick marks is $1^{\prime \prime}$ and a scale bar is shown in panel (d). Panel (a) gives the 3C 212 PSF minus the 3C 245 PSF, both observed in the F675W filter. Panel (b) shows the 3C 2 PSF minus the 3C 212 PSF, in the F675W filter. Panel (C) shows the difference of the 3C 196 PSF minus the 3C 336 PSF, in the F622W filter. Note that the diffraction spikes subtract poorly close to the stellar profile in this case. In Panel (D), we show the average F622W PSF, from the addition of the 3C 196 PSF and the 3C 336 PSFs.

Fig. 2.- PSF Subtraction Method: We show one example of how we determine the (QSO/PSF) scalings in our PSF subtraction procedure. We show in the upper panel the $\chi^{2}$ of the PSF subtraction residuals in an annulus versus the scaling of the subtracted PSF for the $3 \mathrm{C} 2 K^{\prime}$ image. The minimum in this plot gives the scaling for the lower-limit quasar subtraction. In the lower panel we plot the mean flux in an inner and outer annulus versus the PSF scaling; the circles are the outer annulus, the squares are the inner circle. At the intercept of these two lines, the flux in the residual extension will be constant (monotonic) across the entire inner region. This example $\left(3 \mathrm{C} 2\right.$ at $\left.K^{\prime}\right)$ is the quasar image with the greatest contribution of extended material.

Fig. 3.- 3C 2 (quasar). (A) PSF-subtracted HST WFC image (inset shows the same image prior to PSF subtraction). The white cross shows the position of the southern radio hotspot. (B) PSF-subtracted CFHT Redeye $K^{\prime}$ image. Again, the inset shows the unsubtracted version. (C) HST image, enlarged $2 \times$. The inset shows the unsubtracted version with radio contours ( 2 and $32 \mathrm{mJy}_{\text {beam }}{ }^{-1}$ ) from the 2-cm VLA map of Saikia et al. 1987. This does not include the peak of the core flux. (D) Same as (C), but higher contrast. For this and following illustrations, $\mathrm{N}$ is at the top and $\mathrm{E}$ to the left. Tick marks are at $1^{\prime \prime}$ intervals, and long tick marks indicate the source center (optical peak for quasars, $K^{\prime}$ peak for radio galaxies).

Fig. 4.- 3C 175.1 (galaxy). (A) HST WFC image. The crosses indicate the positions of the radio hotspots. (B) Keck NIRC $K^{\prime}$ image. (C) HST image, enlarged $2 \times$, and slightly smoothed to show low-surface-brightness features better. Inset shows lower-contrast image without smoothing. (D) Same as $(A)$, but smoothed and overlayed with contours from our $3.6 \mathrm{~cm}$ VLA superposed. The levels shown are $0.5,2,8,32$, and $128 \mathrm{mJy} \mathrm{beam}^{-1}$. 
Fig. 5.- 3C 196 (quasar). (A) PSF-subtracted HST WFC image. The spiral galaxy $c$ is in the foreground, at $z=0.437$ (Boisse \& Boulade 1990). The crosses indicate the positions of the hotspots, and the inset shows the unsubtracted image. (B) PSF-subtracted Keck NIRC $K^{\prime}$ image, with the inset showing the unsubtracted version. (C) HST image, enlarged $2 \times$. The inset shows the same, with lower contrast. (D) Image obtained with the CFHT SIS fast guider through a $\sim 30 \AA$ interference filter centered on the [O II] $\lambda 3727$ line. The image has been continuum subtracted. The inset shows the same image at lower contrast.

Fig. 6.- 3C 212 (quasar). (A) PSF-subtracted HST WFC image. Note the alignment of objects $a, b, c, f$, and $g$ with the radio axis, indicated by the crosses on the radio hotspot positions. The inset shows the unsubtracted version. (B) PSF-subtracted Keck NIRC $K^{\prime}$ image. (C) HST image, enlarged $2 \times$. (D) Same as $(C)$, but smoothed and at higher contrast.

Fig. 7.- 3C 217 (galaxy). (A) HST WFC image. The cross indicates the approximate position of the $\mathrm{E}$ radio hotspot, and the arrow shows the direction to the $\mathrm{W}$ hotspot, which lies outside the frame. $3 \mathrm{C} 217$ is the only source in our sample that does not have a detected radio core component, so we have assumed the optical position given by Pedelty et al. (1989). (B) Keck NIRC $K^{\prime}$ image. (C) HST image, enlarged $2 \times$, and at lower contrast than $(A)$. The inset shows the image at even lower contrast, to show the faint unresolved core. (D) Same as $(C)$, but smoothed and at higher contrast.

Fig. 8.- 3C 237 (galaxy). (A) HST WFC image. (B) Keck NIRC $K^{\prime}$ image. (C) HST image, enlarged $2 \times$, and at lower contrast than $(A)$. The inset shows the positions of the radio hotspots. (D) Same as $(C)$, but higher contrast.

Fig. 9.- 3C 245 (quasar). (A) PSF-subtracted HST WFC image. The crosses show the positions of the radio hotspots, although the $\mathrm{E}$ lobe is very diffuse, covering much of the region over which low-surface-brightness optical emission is seen. The inset shows the unsubtracted version of the image. (B) PSF-subtracted Keck NIRC $K^{\prime}$ image, with the inset giving the unsubtracted version. (C) HST image, enlarged $2 \times$ and smoothed. The inset shows the enlarged, unsmoothed version at lower contrast. It also shows, displaced $1^{\prime \prime}$ $\mathrm{N}$, radio contours from the $6 \mathrm{~cm}$ map of Laing (1989). Contour levels are 5, 10, 15, 20, 25, $50,100,125,250$, and $375 \mathrm{mJy}^{\text {beam }}{ }^{-1}$. Note the close correspondence between the optical and radio jet structures. (D) Continuum-subtracted [O II] $\lambda 3727$ image through a $\sim 30 \AA$ interference filter at the UH $2.2 \mathrm{~m}$ Telescope. 
Fig. 10.- 3C 280 (galaxy). (A) HST WFC image. The cross shows the position of the $\mathrm{E}$ radio hotspot, and the arrow shows the direction to the $\mathrm{W}$ radio hotspot, which lies outside the frame. The inset shows the same image, smoothed and at higher contrast, in order to show better the low-surface-brightness material in the direction of the $\mathrm{E}$ radio lobe. (B) Keck NIRC $K^{\prime}$ image. The inset shows the same image at lower contrast. (C) HST image, enlarged $2 \times$. The inset shows the same at lower contrast. (D) Image obtained with the CFHT SIS fast guider through a $\sim 30 \AA$ interference filter centered on the [O II] $\lambda 3727$ line. The image has been continuum subtracted and slightly deconvolved. The inset shows the same image at lower contrast.

Fig. 11.- 3C 289 (galaxy). (A) HST WFC image. The crosses show the position of the radio hotspots. (B) Keck NIRC $K^{\prime}$ image. (C) HST image, enlarged $2 \times$. The inset shows the same at lower contrast. (D) Image obtained with the UH 88-inch Telescope through a $\sim 30 \AA$ interference filter centered on the $[\mathrm{O}$ II $] \lambda 3727$ line. The image has been continuum subtracted.

Fig. 12.- 3C 336 (quasar). (A) PSF-subtracted HST WFC image. The arrows show the directions to the radio hotspots, both of which lie outside the frame. The inset shows the unsubtracted image. (B) PSF-subtracted Keck NIRC $K^{\prime}$ image, with the unsubtracted image shown in the inset. (C) HST image, enlarged $2 \times$ and smoothed. (D) Image obtained with the CFHT SIS fast guider through a $\sim 30 \AA$ interference filter centered on the [O II] $\lambda 3727$ line. The image has been continuum subtracted. The inset shows the same image at lower contrast.

Fig. 13.- Photometry in $F_{\lambda}$ of the various components of $3 \mathrm{C} 2$ versus $\lambda_{0}$, normalized by $F_{\lambda}(3300 \AA)$. Filled circles are the QSO nucleus, asterisks are the nebulosity in an annulus from $1^{\prime \prime} .5$ to $3^{\prime \prime}$, unfilled squares are the companion $c$, filled triangles are the northen lobe $a$, and unfilled circles are the faint extension $b$. In panel $A$, the components $a, b$, and $c$ do not have the local background subtracted; in panel $B, a, b$ and $c$ photometry is given minus an estimated local background contribution. The QSO nucleus and nebulosity photometry are identical in the two panels. In panel $B$, we plot models: at the top middle of the figure, we show the SED of M31 from Coleman, Wu \& Weedman (1984) with the alternately shortand long-dashed line; the bluer model (with the same line pattern) is their S0 SED. Dotted lines are Bruzual \& Charlot models: the upper model is a $4 \mathrm{Gyr}$ stellar population, while the next two (in order of brightness at $1 \mu \mathrm{m}$ ) are $2 \mathrm{Gyr}$ and $1 \mathrm{Gyr}$ old. A short-dashed line is a power-law with $\alpha=1.36$. 
Fig. 14.- Photometry of the northern lobe (component $a$ ) with the radio photometry of Saikia et al. (1987) of the same component (crosses). The error bars shown on our data are $2 \sigma$ errors. We plot a linear fit to our data (weighted instrumentally); this gives a power-law with $\alpha=1.36$, where $S_{\nu} \sim \nu^{-\alpha}$. A linear fit to the Saikia et al. points gives $\alpha=0.83$.

Fig. 15.- Our 3C 212 WFPC2 image, with contours from our $3.6 \mathrm{~cm}$ VLA map superposed. The contours are at $-1,1,4,16$ and $256 \mathrm{mJy}_{\text {beam }}{ }^{-1}$.

Fig. 16. - The results of fitting elliptical isophotes to the $K^{\prime} 3 \mathrm{C} 280$ image, with companion objects removed from the fit. In the upper panel, we give the mean surface brightness of each isophote versus the semi-major axis. If a de Vaucouleurs profile is fit to the linear portion of the data, the effective radius is $r_{e}=0^{\prime \prime} 84 \sim 5 \mathrm{kpc}$ corresponding to $r_{e}^{\frac{1}{4}}=0.96$. The inner portion of the profile is smoothed by atmospheric seeing, with seeing FWHM $\approx 0$ " 6 . In the lower panel, we show the PAs of the fit ellipses. 
Fig. 17. - Method used to determine the position angle: the example shown is the position angle on the sky for the Keck $K^{\prime}$ images of $3 \mathrm{C} 175.1$ ( $y$ axis) given as function of the isophotal cutoff level ( $x$ axis; the units are $10^{-19} \mathrm{ergs} \mathrm{cm}^{-2} \mathrm{~s}^{-1} \AA^{-1} \operatorname{arcsec}^{-2}$ ) and as a function of the aperture size used (proportional to the size of the circular points; radii vary from $1^{\prime \prime}$ to 3.75 ). The bright foreground star has been excluded; all other companion objects are included. The two companions centered $\sim 3^{\prime \prime} .5$ to the NE contribute to the moment calculations at low isophotal levels and in apertures of $3^{\prime \prime}$ or more. We adopt $70^{\circ}$ for this position angle by choosing to exclude the companions; the position angle then flattens out at $\sim 70^{\circ}$, before rising again at higher isophotal-level cutoffs.

Fig. 18. - Histograms of the difference between the object PA in the HST image (at a low isophotal level) and the radio axis. The top panel is the sum of the radio galaxy and quasars samples; the middle panel is the quasars alone, and the bottom panel is the radio galaxies alone.

Fig. 19.- Histograms of the difference between the object PA in the HST image (at a high isophotal level, where possible) and the radio axis. The top panel is the sum of the radio galaxy and quasars samples; the middle panel is the quasars alone, and the bottom panel is the radio galaxies alone.

Fig. 20. - Histograms of the difference between the object position angle in the $K^{\prime}$ image and the radio axis. The top panel is the sum of the radio galaxy and quasars samples; the middle panel is the quasars alone, and the bottom panel is the radio galaxies alone.

Fig. 21.- Histograms of the optical-near-infrared spectral index $\alpha$ for the identifiable components whose photometry we present in Table 5 . The top panel gives the values for the quasar nuclei (minus any extension); the middle panel gives the values for discrete companions, while the lower panel represents primarily nebulous material. Many objects (such as $d$ of $3 \mathrm{C} 212$ ) are intermediate between these two classes and are included in both middle and lower histograms.

Fig. 22. - Simulations of the quasars, manufactured by adding a mean quasar "nuclear" PSF (scaled to each of the quasar magnitudes) to each of the observed radio galaxies. 
TABLE 1. Properties of Sample

\begin{tabular}{lccccccccc}
\hline \hline Source & Type & RA (J2000) & DEC (J2000) & Redshift & E $(B-V)^{\mathrm{a}}$ & $\mathrm{S}_{178}(\mathrm{Jy})$ & $\alpha_{2700^{\mathrm{b}}}$ & LAS & Reference \\
\hline 3C 2 & $\mathrm{Q}$ & 000622.59 & -000424.69 & 1.037 & 0.013 & 14.9 & 0.73 & $5^{\prime \prime}$ & 9 \\
3C 175.1 & G & 071404.62 & 143620.55 & 0.920 & 0.128 & 11.4 & 0.93 & $7^{\prime \prime}$ & 6 \\
3C 196 & $\mathrm{Q}$ & 081336.03 & 481302.58 & 0.871 & 0.040 & 68.2 & 0.92 & $6^{\prime \prime}$ & 8 \\
3C 212 & $\mathrm{Q}$ & 085841.43 & 140943.97 & 1.049 & 0.020 & 15.1 & 0.83 & $10^{\prime \prime}$ & 1 \\
3C 217 & $\mathrm{G}$ & 090850.63 & 374818.19 & 0.898 & 0.005 & 11.3 & 1.10 & $12^{\prime \prime}$ & 7 \\
3C 237 & G & 100800.01 & 073016.38 & 0.877 & 0.000 & 20.9 & 0.93 & $1^{\prime \prime} 3$ & 10 \\
3C 245 & Q & 104244.58 & 120331.18 & 1.029 & 0.005 & 14.4 & 0.72 & $8^{\prime \prime}$ & 3 \\
3C 280 & G & 125657.06 & 472019.32 & 0.996 & 0.000 & 23.7 & 0.85 & $13^{\prime \prime}$ & 4 \\
3C 289 & G & 134526.21 & 494632.13 & 0.967 & 0.000 & 12.0 & 0.98 & $10^{\prime \prime}$ & 5 \\
3C 336 & Q & 162439.02 & 234512.72 & 0.927 & 0.035 & 11.5 & 0.91 & $28^{\prime \prime}$ & 2 \\
& & & & & & & & \\
\hline \hline
\end{tabular}

${ }^{a} E(B-V)$ values are derived from Burstein \& Heiles (1984); errors in these values are $\sim 0.015$.

bSpectral index at $2700 \mathrm{MHz}$, where $S_{\nu} \propto \nu^{-\alpha}$. Values have been taken from Herbig \& Readhead (1992) for all sources except $3 \mathrm{C} 2$ and $3 \mathrm{C} 237$, which were calculated from data compiled by Kühr et al. (1981).

References for Table 1.

Sources for radio maps and LAS values: (1) Akujor et al. 1991; (2) Bridle et al. 1994; (3) Laing 1989; (4) Liu \& Pooley 1991; (5) Liu, Pooley, \& Riley 1992; (6) Neff, Roberts, \& Hutchings 1995; (7) Pedelty et al. 1989; (8) Reid et al. 1995; (9) Saikia, Salter, \& Muxlow 1987; (10) van Breugel et al. 1992. 
TABLE 2. HST WFPC2 Observations

\begin{tabular}{llcccc}
\hline \hline Source & Filter & Mean $\lambda_{0}(\AA)$ & $\Delta \lambda_{0}(\AA)$ & Exposure & $\begin{array}{c}\text { Normalized } 1 \sigma \\
\text { Detection Limit }\end{array}$ \\
\hline 3C 2 & F675W & 3298.6 & 437 & $16 \times 1100 \mathrm{~s}$ & 1.82 \\
3C 175.1 & F622W & 3221.4 & 487 & $16 \times 1000 \mathrm{~s}$ & 2.23 \\
3C 196 & F622W & 3305.8 & 500 & $8 \times 900 \mathrm{~s}$ & 2.18 \\
3C 212 & F675W & 3279.2 & 434 & $16 \times 1100 \mathrm{~s}$ & 2.08 \\
3C 217 & F622W & 3258.8 & 493 & $8 \times 900 \mathrm{~s}$ & 1.56 \\
3C 237 & F622W & 3295.2 & 498 & $16 \times 1100 \mathrm{~s}$ & 2.60 \\
3C 245 & F675W & 3311.6 & 438 & $16 \times 1100 \mathrm{~s}$ & 2.18 \\
3C 280 & F622W & 3098.8 & 446 & $8 \times 1100 \mathrm{~s}$ & 2.12 \\
3C 289 & F622W & 3144.5 & 476 & $8 \times 1000 \mathrm{~s}$ & 1.98 \\
3C 336 & F622W & 3209.7 & 485 & $8 \times 900 \mathrm{~s}$ & 2.35 \\
& & & & \\
\hline \hline
\end{tabular}

aThe $1 \sigma$ detection limit in our images, normalized to $z=1$ and $E(B-V)=0$, measured in $10^{-20}$ ergs $\mathrm{cm}^{-2} \mathrm{~s}^{-1} \AA^{-1} \operatorname{arcsec}^{-2}$. 
TABLE 3. Log of Ground-Based Imaging Observations

\begin{tabular}{|c|c|c|c|c|c|c|c|}
\hline Source & Date & Telescope & Detector & Pixel Scale & Filter & Exposure & Data Quality \\
\hline \multirow[t]{7}{*}{$3 \mathrm{C} 2$} & 1992 Nov 26 & UH88 & Tek2048 & $0 \prime 22$ & $7000 / 900$ & $10 \times 600 \mathrm{~s}$ & $0 ! " 86$ \\
\hline & 1993 Sep 21 & UH88 & Tek2048 & $0^{\prime \prime} 22$ & $8964 / 1063$ & $21 \times 300 \mathrm{~s}$ & $0^{\prime \prime} 76$ \\
\hline & 1993 Nov 27 & CFHT & Nic256 & $0^{\prime \prime} \cdot 2$ & $K^{\prime}$ & $27 \times 180 \mathrm{~s}$ & $0 ! 70$ \\
\hline & 1993 Nov 28 & CFHT & $\mathrm{Nic} 256$ & $0^{\prime \prime 2} 2$ & $K^{\prime}$ & $38 \times 180 \mathrm{~s}$ & $0^{\prime \prime} 63$ \\
\hline & 1993 Dec 23 & IRTF & InSb256 & $0^{\prime \prime} 15$ & $K^{\prime}$ & $53 \times 50 \mathrm{~s}$ & $0 ! ' 67$ \\
\hline & 1994 Aug 10 & IRTF & InSb256 & $0 ! 15$ & $H$ & $44 \times 90 \mathrm{~s}$ & $0^{\prime \prime} 78$ \\
\hline & 1994 Nov 21 & UH88 & Qrc1024 & $0 ! 18$ & $H$ & $23 \times 180 \mathrm{~s}$ & $0^{\prime \prime} 97$ \\
\hline \multirow[t]{3}{*}{$3 \mathrm{C} 175.1$} & 1993 Nov 28 & CFHT & Nic256 & $0^{\prime \prime} 2$ & $K^{\prime}$ & $36 \times 180 \mathrm{~s}$ & $0 ! 83$ \\
\hline & 1994 Nov 19 & UH88 & Qrc1024 & $0 ! 18$ & $K^{\prime}$ & $13 \times 180 \mathrm{~s}$ & $1 ! 07$ \\
\hline & 1995 Mar 20 & Keck & InSb256 & $0 ! 15$ & $K^{\prime}$ & $23 \times 120 \mathrm{~s}$ & $0^{\prime \prime} 64$ \\
\hline \multirow[t]{10}{*}{$3 \mathrm{C} 196$} & 1992 Apr 14 & UH88 & Nic256 & $0 ! 37$ & $K^{\prime}$ & $29 \times 90 s$ & 0.68 \\
\hline & 1992 May 07 & UH88 & Tek2048 & $0^{\prime \prime} 22$ & $6968 / 28$ & $2 \times 2400 \mathrm{~s}$ & 1.08 \\
\hline & 1992 May 08 & UH 88 & Tek2048 & $0^{\prime \prime} \cdot 22$ & $6475 / 900$ & $8 \times 600$ & $0 ! 91$ \\
\hline & 1992 May 11 & UH88 & Tek2048 & $0^{\prime \prime} \cdot 22$ & $6968 / 28$ & $2 \times 2400 \mathrm{~s}$ & $1 ! 30$ \\
\hline & 1992 May 10 & CFHT & Nic256 & $0 ! \cdot 3$ & $K^{\prime}$ & $30 \times 90 s$ & $0^{\prime} \cdot 71$ \\
\hline & 1993 Apr 19 & UH88 & Tek2048 & $0 ! 22$ & $6475 / 900$ & $9 \times 600 \mathrm{~s}$ & $0 ! 83$ \\
\hline & 1994 Mar 27 & UH88 & Nic256 & $0 ! 37$ & $K^{\prime}$ & $51 \times 90 \mathrm{~s}$ & $0^{\prime \prime} 97$ \\
\hline & 1994 Apr 05 & UH88 & Tek2048 & $0^{\prime \prime} .22$ & $6120 / 960$ & $6 \times 600 \mathrm{~s}$ & $1 ! 15$ \\
\hline & 1995 Feb 24 & CFHT & Orb2048 & 0.087 & $6983 / 32$ & $3 \times 2400 \mathrm{~s}$ & $0 ! .60$ \\
\hline & $1995 \mathrm{Mar} 19$ & Keck & InSb256 & $0 \prime 15$ & $K^{\prime}$ & $27 \times 96 \mathrm{~s}$ & $0 ! .85$ \\
\hline \multirow[t]{7}{*}{$3 \mathrm{C} 212$} & 1992 May 11 & CFHT & Nic256 & 0.3 & $K^{\prime}$ & $37 \times 90 \mathrm{~s}$ & $0 ! 61$ \\
\hline & $1993 \mathrm{Apr} 17$ & UH88 & Tek2048 & $0 ! 22$ & $7000 / 900$ & $7 \times 900 \mathrm{~s}$ & $0 ! 81$ \\
\hline & 1993 Dec 23 & IRTF & InSb256 & $0^{\prime \prime} 15$ & $K^{\prime}$ & $126 \times 50 \mathrm{~s}$ & $0 ! ' 83$ \\
\hline & 1994 May 04 & UH88 & Nic256 & $0 ! 12$ & $K^{\prime}$ & $10 \times 360 \mathrm{~s}$ & $0 ! ' 88$ \\
\hline & 1995 Jan 17 & UH88 & Qrc1024 & $0 .: 06$ & $K^{\prime}$ & $25 \times 300 \mathrm{~s}$ & $0 ! 53$ \\
\hline & 1995 Feb 24 & CFHT & Orb2048 & $0^{\prime \prime} 087$ & $7646 / 32$ & $3 \times 2400 \mathrm{~s}$ & $0 ! .80$ \\
\hline & 1995 Mar 20 & Keck & $\operatorname{InSb} 256$ & 0.15 & $K^{\prime}$ & $27 \times 120 \mathrm{~s}$ & $0 ! 67$ \\
\hline $3 \mathrm{C} 217$ & 1995 Mar 19 & Keck & InSb256 & $0 " 15$ & $K^{\prime}$ & $18 \times 120 \mathrm{~s}$ & $0 ! .83$ \\
\hline \multirow[t]{2}{*}{$3 \mathrm{C} 237$} & 1994 Nov 22 & UH88 & Qrc1024 & $0 ! 18$ & $K^{\prime}$ & $14 \times 180 \mathrm{~s}$ & $0^{\prime \prime} 78$ \\
\hline & 1995 Mar 20 & Keck & InSb256 & $0 ! 15$ & $K^{\prime}$ & $15 \times 120 \mathrm{~s}$ & $0 ! .85$ \\
\hline \multirow[t]{5}{*}{$3 \mathrm{C} 245$} & 1992 May 11 & CFHT & Nic256 & $0 \prime 3$ & $K^{\prime}$ & $18 \times 90 \mathrm{~s}$ & $0 ! 74$ \\
\hline & 1993 Apr 12 & UH88 & Nic256 & $0: 37$ & $K^{\prime}$ & $50 \times 90 \mathrm{~s}$ & $1 " 11$ \\
\hline & 1993 Apr 16 & UH 88 & Tek2048 & $0^{\prime \prime} 22$ & $6475 / 900$ & $10 \times 600 \mathrm{~s}$ & $0 ! 80$ \\
\hline & 1994 Apr 06 & UH88 & Tek2048 & $0^{\prime \prime} 22$ & $7571 / 31$ & $3 \times 1800 \mathrm{~s}$ & $0^{\prime \prime} 75$ \\
\hline & 1995 Mar 20 & Keck & InSb256 & $0 ! 15$ & $K^{\prime}$ & $18 \times 120 \mathrm{~s}$ & $1{ }^{\prime \prime} 33$ \\
\hline \multirow[t]{4}{*}{$3 \mathrm{C} 280$} & 1994 Apr 06 & UH88 & Tek2048 & $0 ! 22$ & $7449 / 31$ & $3 \times 1800 \mathrm{~s}$ & $0^{\prime \prime} 73$ \\
\hline & 1994 May 04 & UH88 & Nic256 & $0 . \prime 12$ & $K^{\prime}$ & $23 \times 360 \mathrm{~s}$ & $0 \prime 90$ \\
\hline & 1995 Feb 23 & CFHT & Orb2048 & 0.087 & $7449 / 31$ & $4 \times 2700 \mathrm{~s}$ & $0 ! 66$ \\
\hline & 1995 Mar 19 & Keck & InSb256 & $0 ! ! 15$ & $K^{\prime}$ & $27 \times 96 s$ & $0^{\prime \prime} 58$ \\
\hline \multirow[t]{3}{*}{$3 \mathrm{C} 289$} & 1994 Apr 06 & UH 88 & Tek2048 & $0^{\prime \prime} 22$ & $7388 / 29$ & $3 \times 1800 \mathrm{~s}$ & $0 ! .81$ \\
\hline & 1995 Mar 19 & Keck & InSb256 & $0 ! 15$ & $K^{\prime}$ & $20 \times 120 \mathrm{~s}$ & $0 ! 78$ \\
\hline & 1995 Mar 20 & Keck & InSb256 & 0.15 & $K^{\prime}$ & $24 \times 120 \mathrm{~s}$ & $0 ! 80$ \\
\hline \multirow[t]{10}{*}{$3 \mathrm{C} 336$} & 1992 Apr 13 & UH88 & $\mathrm{Nic} 256$ & $0 ! 37$ & $K^{\prime}$ & $12 \times 90 \mathrm{~s}$ & $0 ! 70$ \\
\hline & $1992 \mathrm{Apr} 14$ & UH88 & Nic256 & 0.37 & $K^{\prime}$ & $30 \times 90 \mathrm{~s}$ & $0 ! 75$ \\
\hline & 1992 May 07 & UH88 & Tek2048 & $0 ! 22$ & $6475 / 900$ & $5 \times 600 \mathrm{~s}$ & $0^{\prime \prime} 94$ \\
\hline & 1992 May 10 & CFHT & Nic256 & $0^{\prime \prime} 3$ & $K^{\prime}$ & $33 \times 90 s$ & $0 ! 76$ \\
\hline & 1993 Apr 17 & UH88 & Tek2048 & $0 ! 22$ & $6475 / 900$ & $8 \times 600 \mathrm{~s}$ & $0 ! 91$ \\
\hline & 1994 Apr 05 & UH88 & Tek2048 & $0 ! 22$ & $7192 / 28$ & $2 \times 2100 \mathrm{~s}$ & $1 ! .03$ \\
\hline & 1994 May 02 & IRTF & $\operatorname{InSb} 256$ & $0^{\prime \prime} 31$ & $K^{\prime}$ & $144 \times 36 \mathrm{~s}$ & $1 " 30$ \\
\hline & 1994 May 03 & UH88 & Nic256 & $0^{\prime \prime} 12$ & $K^{\prime}$ & $9 \times 360 \mathrm{~s}$ & $0^{\prime \prime} 72$ \\
\hline & 1995 Feb 24 & CFHT & Orb2048 & 0.087 & $7192 / 28$ & $3 \times 2100 \mathrm{~s}$ & $0 ! 64$ \\
\hline & 1995 Mar 19 & Keck & InSb256 & 0.15 & $K^{\prime}$ & $27 \times 96 \mathrm{~s}$ & $0 ! 449$ \\
\hline
\end{tabular}


TABLE 4. PSF Star Observations

\begin{tabular}{lccccc}
\hline \hline Quasar Name & Star Name & Filter & $\begin{array}{c}\text { Integration } \\
\text { Time }\end{array}$ & $\begin{array}{c}\text { QSO Saturation } \\
\text { Diameter }\end{array}$ & $\begin{array}{c}\text { PSF Saturation } \\
\text { Diameter }\end{array}$ \\
\hline 3C 2 & GSC4663-00565 & F675W & $12 \times 4 \mathrm{~s}$ & $0^{\prime \prime} 00$ & $0^{\prime \prime} 25$ \\
3C 196 & GSC3411-02379 & F622W & $12 \times 4 \mathrm{~s}$ & $0^{\prime \prime} 20$ & $0^{\prime \prime} 05$ \\
3C 212 & GSC0817-02062 & F675W & $12 \times 16 \mathrm{~s}$ & $0^{\prime \prime} 00$ & $0^{\prime \prime} 05$ \\
3C 245 & GSC0842-00459 & F675W & $12 \times 3 \mathrm{~s}$ & $0^{\prime \prime} 25$ & $0^{\prime \prime} 00$ \\
3C 336 & $\cdots$ & F622W & $12 \times 50 \mathrm{~s}$ & $0^{\prime \prime} 20$ & $0^{\prime \prime} 10$ \\
\hline \hline
\end{tabular}


TABlE 5. Optical and Near-Infrared Component Photometry

\begin{tabular}{|c|c|c|c|c|c|c|}
\hline Source & Component & Aperture Type & Aperture Area ${ }^{b}$ & $F_{\lambda}(0.33 \mu \mathrm{m})^{c}$ & $F_{\lambda}(1 \mu \mathrm{m})^{\mathrm{d}}$ & $\alpha(\text { opt })^{e}$ \\
\hline \multirow[t]{2}{*}{$3 C 175.1$} & $a$ & $\mathrm{I}(2.7)$ & 0.77 & $47.5 \pm 2.3$ & $\ldots$ & $\ldots$ \\
\hline & Core & $C(4.5)$ & 63.6 & $80.0 \pm 8$ & $<150$ & $\ldots$ \\
\hline \multirow[t]{5}{*}{ 3C 196} & QSO & $C(5)$ & 80.1 & $17649.0 \pm 350$ & $4285.0 \pm 70$ & 0.84 \\
\hline & $a$ & $\mathrm{R}(3.2,1.65)$ & 5.3 & $276.2 \pm 5$ & $115.4 \pm 6$ & 1.29 \\
\hline & $b$ & $C(0.25)$ & 0.20 & $40.4 \pm 2$ & $\ldots$ & $\ldots$ \\
\hline & $d$ & $C(1.0)$ & 3.1 & $107.0 \pm 10$ & $9.8 \pm 2$ & $\ldots$ \\
\hline & $e$ & $C(1.0)$ & 3.1 & $73.6 \pm 8$ & $8.7 \pm 1.4$ & $\cdots$ \\
\hline \multirow[t]{11}{*}{$3 C 212$} & QSO & $\mathrm{C}(4)$ & 50.3 & $4726.0 \pm 60$ & $2365.0 \pm 30$ & 1.39 \\
\hline & $a$ & $C(0.25)$ & 0.20 & $11.4 \pm 1.9$ & $\ldots$ & $\cdots$ \\
\hline & $b$ & $C(0.35)$ & 0.38 & $20.1 \pm 3.5$ & $\ldots$ & $\ldots$ \\
\hline & $c$ & $\mathrm{C}(0.30)$ & 0.28 & $8.2 \pm 2.1$ & $\cdots$ & $\cdots$ \\
\hline & $b+c$ & $R(1.55,1.7)$ & 26.4 & $48.5 \pm 1.8$ & $25.0 \pm 6$ & 1.42 \\
\hline & $d$ & $\mathrm{R}(2.05,1.2)$ & 2.46 & $39.6 \pm 3.5$ & $18.8 \pm 2.7$ & 1.35 \\
\hline & $e$ & $\mathrm{C}(0.45)$ & 0.64 & $8.9 \pm 1.8$ & $12.1 \pm 1.8$ & 2.27 \\
\hline & $f$ & $R(2.65,3.6)$ & 9.54 & $240.7 \pm 10.5$ & $146.6 \pm 20.9$ & 1.57 \\
\hline & $g$ & $C(1.5)$ & 7.1 & $37.6 \pm 3$ & $71.2 \pm 4.2$ & 2.56 \\
\hline & $h$ & $\mathrm{I}(0.4)^{f}$ & 2.5 & $40.7 \pm 2$ & $8.6 \pm 0.9$ & 0.64 \\
\hline & Extension & $A(1,2.65)$ & 18.9 & $182.2 \pm 10$ & $128.6 \pm 20$ & 1.69 \\
\hline \multirow[t]{3}{*}{$3 C 217$} & $a$ & $\mathrm{C}(1.0)$ & 3.1 & $16.1 \pm 1.3$ & $52.7 \pm 4.3$ & 2.97 \\
\hline & $c$ & $C(1.0)$ & 3.1 & $22.2 \pm 6.5$ & $2.6 \pm 1.9$ & 0.25 \\
\hline & Core & $C(4.5)$ & 63.6 & $70.2 \pm 5$ & $<168$ & $\ldots$ \\
\hline \multirow[t]{3}{*}{$3 \mathrm{C} 237$} & East & $\mathrm{R}(0.95,0.85)$ & 0.81 & $187.8 \pm 1.2$ & $\cdots$ & $\cdots$ \\
\hline & West & $R(0.95,0.9)$ & 0.86 & $213.0 \pm 1.3$ & $\ldots$ & $\ldots$ \\
\hline & Core & $\mathrm{C}(4.5)$ & 63.6 & $162.1 \pm 4.3$ & $<199$ & $\ldots$ \\
\hline \multirow[t]{7}{*}{$3 C 245$} & QSO & $C(4)$ & 50.3 & $21228.0 \pm 140$ & $3468.0 \pm 200$ & 0.41 \\
\hline & $a$ & $C(0.25)$ & 0.20 & $22.3 \pm 1.1$ & $\ldots$ & $\cdots$ \\
\hline & $b$ & $C(0.25)$ & 0.20 & $8.7 \pm 1.1$ & $\cdots$ & $\cdots$ \\
\hline & $a+b($ jet $)$ & $I(3.6)$ & 0.42 & $46.1 \pm 1.9$ & $\cdots$ & $\cdots$ \\
\hline & $c$ & $\mathrm{I}(3.6)$ & 0.22 & $10.9 \pm 1$ & $\ldots$ & $\ldots$ \\
\hline & $d$ & $I(3.6)$ & 0.79 & $38.2 \pm 2.8$ & $\ldots$ & $\cdots$ \\
\hline & $e$ & $C(3.5)$ & 15.9 & $423.2 \pm 6$ & $879.0 \pm 47$ & 2.64 \\
\hline \multirow[t]{5}{*}{$3 C 280$} & $a+b$ & $\mathrm{I}(5.2)$ & 0.56 & $197.2 \pm 2$ & $\ldots$ & $\ldots$ \\
\hline & $c$ & $\mathrm{I}(5.2)$ & 0.56 & $96.0 \pm 2$ & $\ldots$ & $\ldots$ \\
\hline & $d$ & $\mathrm{I}(5.2)$ & 0.78 & $96.7 \pm 2$ & $\ldots$ & $\cdots$ \\
\hline & Elliptical & $C(4)$ & 50.3 & $191.0 \pm 40$ & $608.0 \pm 30$ & 2.95 \\
\hline & Core & $\mathrm{C}(4.5)$ & 63.6 & $164.3 \pm 5$ & $<69$ & $\ldots$ \\
\hline \multirow[t]{6}{*}{$3 C 289$} & $a$ & $C(0.35)$ & 0.38 & $9.6 \pm 2$ & $\cdots$ & $\ldots$ \\
\hline & $b$ & $C(0.35)$ & 0.38 & $7.4 \pm 2$ & $\cdots$ & $\ldots$ \\
\hline & $c$ & $\mathrm{I}(5.2)$ & 0.42 & $17.2 \pm 1.6$ & $\ldots$ & $\cdots$ \\
\hline & $d$ & $C(1)$ & 3.1 & $11.8 \pm 3$ & $22.6 \pm 5.4$ & 2.53 \\
\hline & Elliptical & $\mathrm{C}(3.2)$ & 32.2 & $259.0 \pm 31$ & $694.0 \pm 60$ & 2.89 \\
\hline & Core & $C(4.5)$ & 63.6 & $117.1 \pm 8$ & $<140$ & $\cdots$ \\
\hline \multirow[t]{5}{*}{$3 C 336$} & QSO & $C(4)$ & 50.3 & $20705.0 \pm 414$ & $2216.0 \pm 60$ & 0.17 \\
\hline & $d$ & $\mathrm{R}(1.4,2.95)$ & 4.1 & $79.6 \pm 9.6$ & $63.7 \pm 9.6$ & 1.82 \\
\hline & $a$ & $\mathrm{R}(1.15,0.75)$ & 0.86 & $44.1 \pm 4.8$ & $10.4 \pm 1.4$ & 0.82 \\
\hline & $b$ & $\mathrm{C}(0.6)$ & 1.1 & $26.3 \pm 3.6$ & $11.4 \pm 1.2$ & 1.32 \\
\hline & $c$ & $C(0.6)$ & 1.1 & $36.1 \pm 4.8$ & $13.2 \pm 1.8$ & 1.18 \\
\hline
\end{tabular}

Aperture type; i.e., $\mathrm{C}(r)$ is circular with radius $r ; \mathrm{A}(r 1, r 2)$ is annular with inner radius $r 1$ and outer radius $r 2 ; \mathrm{R}(x, y)$ is rectangular with dimensions $x$ and $y$; and $\mathrm{I}(\mu)$ is an isophotal aperture with surface brightness cutoff level $\mu ; r, r 1, r 2, x$, and $y$ are all in arcseconds and $\mu$ is in $10^{-19} \mathrm{ergs} \mathrm{cm}^{-2} \mathrm{~s}^{-1} \AA^{-1}$ arcsec ${ }^{-2}$, at the wavelength of the HST image. This value is normalized by the correction to $z=1$ factor.

bAperture areas in square arcseconds.

' Background subtracted flux densities from the HST image, in units of $10^{-20} \mathrm{ergs} \mathrm{cm}^{-2} \mathrm{~s}^{-1} \AA^{-1}$. The HST rest wavelength for each object is given in Table 2. Flux densities have been corrected for galactic extinction, but not normalized to $z=1$.

${ }^{d}$ Background subtracted flux densities from the $K^{\prime}$ image, in units of $10^{-20} \mathrm{ergs}_{\mathrm{cm}}^{-2} \mathrm{~s}^{-1} \AA^{-1}$. Fluxes have not been normalized to $z=1$.

eSpectral index from $0.33 \mu \mathrm{m}$ to $1 \mu \mathrm{m} ; F_{\nu} \propto \nu^{-\alpha}$

'Cutoff level is in Gaussian-smoothed HST image. 
TABLE 6. 3C 2 Component Photometry

\begin{tabular}{|c|c|c|c|c|c|c|c|c|}
\hline \multirow[b]{2}{*}{ Component } & \multirow[b]{2}{*}{$\Delta \mathrm{RA}$} & \multirow[b]{2}{*}{$\triangle \mathrm{DEC}$} & \multirow[b]{2}{*}{ Aperture $^{\mathrm{a}}$} & \multirow[b]{2}{*}{$\lambda_{0}(\AA)^{\mathbf{b}}$} & \multicolumn{2}{|c|}{ Subtracted $^{c}$} & \multicolumn{2}{|c|}{ Unsubtracted $^{\circ}$} \\
\hline & & & & & $F_{\lambda}$ & $\sigma\left(F_{\lambda}\right)$ & $F_{\lambda}$ & $\sigma\left(F_{\lambda}\right)$ \\
\hline \multirow[t]{4}{*}{$a$ (NE “lobe") } & 0.17 & $0,: 80$ & $C(0.75)$ & 3299 & 101.3 & 5. & 125.0 & 5. \\
\hline & & & & 4401 & 60.2 & 12. & 75.7 & 12. \\
\hline & & & & 8100 & 57.5 & 9. & 90.3 & 9. \\
\hline & & & & 10309 & 48.4 & 5. & 77.6 & 5. \\
\hline \multirow[t]{4}{*}{$b$ (NW Extension) } & $-0 ! 53$ & 0 ".13 & $\mathrm{R}(0.75,0.75)$ & 3299 & 15.6 & 2.7 & 29.2 & 2.7 \\
\hline & & & & 4401 & 6.7 & 3.6 & 12.8 & 3.6 \\
\hline & & & & 8100 & 3.1 & 6.4 & 17.3 & 6.4 \\
\hline & & & & 10309 & 10.7 & 2.7 & 24.6 & 2.7 \\
\hline \multirow[t]{4}{*}{$c$ (SE Companion) } & 0.80 & -1.06 & $C(0.75)$ & 3299 & 21.0 & 2.7 & 30.1 & 1.8 \\
\hline & & & & 4401 & 45.6 & 9.1 & 85.8 & 9.1 \\
\hline & & & & 8100 & 57.5 & 6.4 & 80.3 & 6.4 \\
\hline & & & & 10309 & 55.7 & 4.6 & 94.9 & 4.6 \\
\hline \multirow[t]{4}{*}{ Symmetric Nebulosity } & $\cdots$ & $\cdots$ & $\mathrm{A}(1.75,3.0)$ & 3299 & 76.6 & 6.4 & $\cdots$ & $\cdots$ \\
\hline & & & & 4401 & 95.8 & 31.9 & $\ldots$ & $\cdots$ \\
\hline & & & & 8100 & 147.8 & 41.1 & $\cdots$ & $\cdots$ \\
\hline & & & & 10309 & 104.9 & 7.3 & $\cdots$ & $\cdots$ \\
\hline \multirow[t]{4}{*}{ QSO (without extensions) } & 0.00 & $0 . \prime 00$ & $\mathrm{C}(4)$ & 3299 & 1579.4 & 18 & $\cdots$ & $\cdots$ \\
\hline & & & & 4401 & 1367.0 & 59. & $\cdots$ & $\cdots$ \\
\hline & & & & 8100 & 609.5 & 46. & $\cdots$ & $\cdots$ \\
\hline & & & & 10309 & 514.0 & 27. & $\cdots$ & $\cdots$ \\
\hline
\end{tabular}

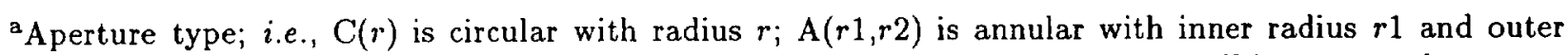
radius $r 2 ; \mathrm{R}(x, y)$ is rectangular with dimensions $x$ and $y$; and $r, r 1, r 2, x$, and $y$ are all in arcseconds.

b This is the rest wavelength in $\AA$, assuming each component has the same redshift as the narrow-line $3 \mathrm{C} 2$ redshift of 1.037 .

cFor the components $a, b$, and $c$, these fluxes have had the best estimate of the local background due to the nebulosity subtracted; see the text for details. For the QSO itself, all extensions have been subtracted. Flux densities are in units of $10^{-20}$ ergs cm${ }^{-2} \mathrm{~s}^{-1} \AA^{-1}$ and have been corrected for galactic extinction.

$d$ These fluxes have had no local background subtracted (other than standard sky subtraction). Flux densities are in units of $10^{-20} \mathrm{ergs} \mathrm{cm}^{-2} \mathrm{~s}^{-1} \AA^{-1}$ and have been corrected for galactic extinction. 


\section{TABle 7. Quasar Optical Photometry}

\begin{tabular}{|c|c|c|c|c|c|}
\hline Source $^{a}$ & Aperture $^{b}$ & Normalized Cutoff ${ }^{c}$ & $F_{\lambda}(\operatorname{mon})^{\mathrm{d}}$ & $F_{\lambda}\left(\chi^{2} \min \right)^{\mathbf{e}}$ & Notes $^{f}$ \\
\hline $3 \mathrm{C} 2$ & $\mathrm{~A}(0.3,3.75)$ & $\mathrm{I}(4.56)$ & 243.1 & 230.2 & $c$ included; $\Delta F=23$ \\
\hline $3 \mathrm{C} 2$ & $\mathrm{~A}(0.3,3.75)$ & None & 484.8 & 469.3 & $c$ included; $\Delta F=21$ \\
\hline $3 \mathrm{C} 2$ (int) & $\mathrm{A}(0,3.75)$ & $\mathrm{I}(4.56)$ & 275.5 & 253.8 & $c$ included; $\Delta F=23$ \\
\hline $3 \mathrm{C} 2$ (int) & $\mathrm{A}(0,3.75)$ & None & 528.7 & 505.2 & $c$ included; $\Delta F=21$ \\
\hline $3 \mathrm{C} 196$ & $\mathrm{~A}(0.45,3.75)$ & $\mathrm{I}(4.56)$ & 457.2 & 352.1 & foreground $c$ excluded \\
\hline $3 \mathrm{C} 196$ & $\mathrm{~A}(0.45,3.75)$ & None & 578.2 & 504.7 & foreground $c$ excluded \\
\hline 3C 196 (int) & $\mathrm{A}(0,3.75)$ & $\mathrm{I}(4.56)$ & 535.9 & 427.3 & foreground $c$ excluded \\
\hline 3C 196 (int) & $\mathrm{A}(0,3.75)$ & None & 699.5 & 579.9 & foreground $c$ excluded \\
\hline $3 \mathrm{C} 212$ & $\mathrm{~A}(0.45,3.75)$ & $\mathrm{I}(4.56)$ & 108.7 & $\cdots$ & $h$ included; $\Delta F=5$ \\
\hline $3 \mathrm{C} 212$ & $\mathrm{~A}(0.45,3.75)$ & None & 338.2 & $\cdots$ & $h$ included; $\Delta F=21$ \\
\hline $3 \mathrm{C} 212$ (int) & $\mathrm{A}(0,3.75)$ & $\mathrm{I}(4.56)$ & 136.4 & $(86.9)^{\mathrm{g}}$ & $h$ included; $\Delta F=5$ \\
\hline $3 \mathrm{C} 212$ (int) & $\mathrm{A}(0,3.75)$ & None & 371.1 & $(362.2)^{\mathrm{g}}$ & $h$ included; $\Delta F=21$ \\
\hline $3 \mathrm{C} 245$ & $\mathrm{~A}(0.45,3.75)$ & $\mathrm{I}(4.56)$ & 138.6 & $(88.9)^{h}$ & $e$ excluded \\
\hline $3 \mathrm{C} 245$ & $\mathrm{~A}(0.45,3.75)$ & None & 325.6 & $(272.1)^{\mathrm{h}}$ & $e$ excluded \\
\hline $3 \mathrm{C} 245$ (int) & $\mathrm{A}(0,3.75)$ & $\mathrm{I}(4.56)$ & 202.4 & $\ldots$ & $e$ excluded \\
\hline $3 \mathrm{C} 245$ (int) & $\mathrm{A}(0,3.75)$ & None & 389.4 & $(313.5)^{f}$ & $e$ excluded \\
\hline $3 \mathrm{C} 336$ & $\mathrm{~A}(0.55,3.75)$ & $I(4.56)$ & 84.6 & 43.9 & foreground $d$ excluded \\
\hline 3C 336 & $\mathrm{~A}(0.55,3.75)$ & None & 263.5 & 140.6 & foreground $d$ excluded \\
\hline $3 C 336$ (int) & $\mathrm{A}(0.55,3.75)$ & None & 291.7 & $\cdots$ & foreground $d$ excluded \\
\hline
\end{tabular}

${ }^{a}$ Quasar name; if followed by (int), this indicates that the central region of the quasar has been interpolated with a low-order polynomial fit.

${ }^{\mathrm{b}}$ Aperture size; $\mathrm{A}(r 1, r 2)$ signifies annular aperture with inner radius $r 1$ and outer radius $r 2$, where $r 1$ and $r 2$ are in arcseconds.

'Isophotal cutoff level; $\mathrm{I}(\mu)$ signifies a surface brightness cutoff level of $\mu$ in $10^{-19} \mathrm{ergs} \mathrm{cm}^{-2} \mathrm{~s}^{-1} \AA^{-1}$ $\operatorname{arcsec}^{-2}$, at the wavelength of the HST image. This surface brightness value has been normalized to $z=1$.

dFlux densities from the monotonic PSF subtraction limit, in units of $10^{-20}$ ergs $\mathrm{cm}^{-2} \mathrm{~s}^{-1} \AA^{-1}$. Flux densities have been corrected for galactic extinction, and normalized to $z=1$.

'Flux densities from the $\chi^{2}$ minimum PSF subtraction limit, in units of $10^{-20} \mathrm{ergs}^{\mathrm{cm}} \mathrm{cm}^{-2} \mathrm{~s}^{-1} \AA^{-1}$. Flux densities have been corrected for galactic extinction, and normalized to $z=1$. Where the $\chi^{2}$ minimum and the monotonic subtraction limit agree, this value is placed in the monotonic-limit column only. Values in parentheses are "lower limit" flux densities calculated by another method, and are placed here for convenience. See notes associated with individual values.

'We note here whether nearby companions were included or not. We included all companions except the foreground spiral $c$ in the field 3C 196, the elliptical $e$ in $3 \mathrm{C} 245$, and the foreground $d$ in the field of 3C 336. For those objects in which we included a nearby (perhaps discrete) companion, we give the change in flux density that would result from subtracting that companion's contribution.

${ }^{8}$ This lower-limit value was derived by replacing the central 1 ".25 $\times 0$ ".95 region with a constant; this excluded high surface brightness regions immediately exterior to the PSF subtraction annulus.

h This lower-limit value was derived by excluding the central $0^{\prime \prime} 65$ instead of the standard 0 . 45 ; this certainly excludes real flux, but also excludes PSF residuals near the edge of the PSF subtraction region. 
Table 8. Radio Galaxy Optical Photometry

\begin{tabular}{|c|c|c|c|c|}
\hline Source & Aperture $^{a}$ & $F_{\lambda}(\text { isophotal })^{\mathbf{b}}$ & $F_{\lambda}(\text { total })^{c}$ & Notes \\
\hline \multirow[t]{2}{*}{$3 \mathrm{C} 175.1$} & $\mathrm{~A}(0,3.0)$ & 154.5 & 355.3 & d \\
\hline & $\mathrm{A}(0.45,3.0)$ & 38.1 & 236.4 & d \\
\hline \multirow[t]{4}{*}{$3 \mathrm{C} 217$} & $\mathrm{~A}(0,3.75)$ & 211.9 & 373.7 & \\
\hline & $\mathrm{A}(0.45,3.75)$ & 83.9 & 244.8 & \\
\hline & $\mathrm{A}(0,3.75)$ & 191.9 & 343.1 & e \\
\hline & $\mathrm{A}(0.45,3.75)$ & 63.8 & 215.0 & e \\
\hline \multirow[t]{2}{*}{$3 \mathrm{C} 237$} & $\mathrm{~A}(0,3.0)$ & 472.5 & 607.2 & \\
\hline & $\mathrm{A}(0.45,3.0)$ & 183.6 & 316.5 & \\
\hline \multirow[t]{3}{*}{$3 \mathrm{C} 280$} & $\mathrm{~A}(0,6.0)$ & 581.9 & 915.9 & f \\
\hline & $\mathrm{A}(0,3.75)$ & 513.5 & 682.0 & $\mathrm{fg}_{\mathrm{g}}$ \\
\hline & $\mathrm{A}(0.45,3.75)$ & 280.3 & 494.7 & $\mathrm{fg}_{\mathrm{g}}$ \\
\hline \multirow[t]{3}{*}{$3 \mathrm{C} 289$} & $\mathrm{~A}(0,3.75)$ & 169.8 & 391.6 & \\
\hline & $\mathrm{A}(0,3.75)$ & 160.8 & 374.2 & h \\
\hline & $\mathrm{A}(0.45,3.75)$ & 36.1 & 247.4 & $\mathbf{h}$ \\
\hline
\end{tabular}

${ }^{a}$ Aperture size; $\mathrm{A}(r 1, r 2)$ signifies annular aperture with inner radius $r 1$ and outer radius $r 2$, where $r 1$ and $r 2$ are in arcseconds.

${ }^{b}$ Flux densities in units of $10^{-20}$ ergs $\mathrm{cm}^{-2} \mathrm{~s}^{-1} \AA^{-1}$, above an isophotal cutoff level of $4.56 \times 10^{-19} \mathrm{ergs} \mathrm{cm}^{-2} \mathrm{~s}^{-1} \AA^{-1} \operatorname{arcsec}^{-2}$, at the wavelength of the HST image, normalized to $\mathrm{E}(B-V)=0$, $z \overline{\bar{c}}$ Total flux densities in aperture, with no isophotal cutoff, in units of $10^{-20}$ ergs $\mathrm{cm}^{-2} \mathrm{~s}^{-1} \AA^{-1}$. Flux densities have been corrected for galactic extinction, and normalized to $z=1$.

${ }^{\mathrm{d}}$ Foreground star removed.

${ }^{\mathrm{e}}$ Components $a$ and $c$ excluded.

${ }^{f}$ Foreground galaxy removed.

${ }^{g}$ Companions, eastern lobe region excluded.

hComponent $d$ excluded. 


\section{TABLE 9. Quasar Near-Infrared Photometry}

\begin{tabular}{lccccl}
\hline \hline \multicolumn{1}{c}{ Source $^{\mathrm{a}}$} & Aperture $^{\mathrm{b}}$ & Normalized Cutoff $^{\mathrm{c}}$ & $F_{\lambda}(\mathrm{mon})^{\mathrm{d}}$ & $F_{\lambda}\left(\chi^{2} \mathrm{~min}\right)^{\mathrm{e}}$ & Notes $^{\mathrm{f}}$ \\
\hline 3C 2 & $\mathrm{A}(0.4,3.75)$ & $\mathrm{I}(1.40)$ & 427.3 & 391.3 & $c$ included; $\Delta F=39$ \\
3C 2 & $\mathrm{A}(0.4,3.75)$ & None & 515.6 & 470.1 & $c$ included; $\Delta F=39$ \\
3C 2 (int) & $\mathrm{A}(0,3.75)$ & None & 554.1 & $\ldots$ & $c$ included; $\Delta F=39$ \\
3C 2 (int) & $\mathrm{A}(0,3.75)$ & $\mathrm{I}(1.40)$ & 465.8 & $\ldots$ & $c$ included; $\Delta F=39$ \\
3C 196 & $\mathrm{A}(0.9,3.75)$ & $\mathrm{I}(1.40)$ & 73.5 & 67.8 & foreground $c$ excluded \\
3C 196 & $\mathrm{A}(0.9,3.75)$ & None & 131.8 & 123.9 & foreground $c$ excluded \\
3C 196 (int) & $\mathrm{A}(0,3.75)$ & $\mathrm{I}(1.40)$ & 75.1 & 67.8 & foreground $c$ excluded \\
3C 196 (int) & $\mathrm{A}(0,3.75)$ & None & 148.8 & 142.3 & foreground $c$ excluded \\
3C 212 & $\mathrm{A}(0.8,3.75)$ & $\mathrm{I}(1.40)$ & 143.6 & 130.0 & $h$ included; $\Delta F=9$ \\
3C 212 & $\mathrm{A}(0.8,3.75)$ & None & 278.7 & 265.7 & $h$ included; $\Delta F=9$ \\
3C 336 & $\mathrm{A}(0.85,3.75)$ & $\mathrm{I}(1.40)$ & 26.4 & 25.4 & foreground $d$ excluded \\
3C 336 & $\mathrm{A}(0.85,3.75)$ & None & 84.5 & 82.1 & foreground $d$ excluded \\
3C 336 (int) & $\mathrm{A}(0,3.75)$ & None & 93.7 & $\cdots$ & foreground $d$ excluded
\end{tabular}

${ }^{a}$ Quasar name; if followed by (int), this indicates that the central region of the quasar has been interpolated with a low-order polynomial fit.

${ }^{b}$ Aperture size; $\mathrm{A}(r 1, r 2)$ signifies annular aperture with inner radius $r 1$ and outer radius $r 2$, where $r 1$ and $r 2$ are in arcseconds.

${ }^{c}$ Isophotal cutoff level; $\mathrm{I}(\mu)$ signifies a surface brightness cutoff level of $\mu$ in $10^{-19} \mathrm{ergs} \mathrm{cm}^{-2} \mathrm{~s}^{-1} \AA^{-1}$ $\operatorname{arcsec}^{-2}$. This surface brightness value has been normalized to $z=1$.

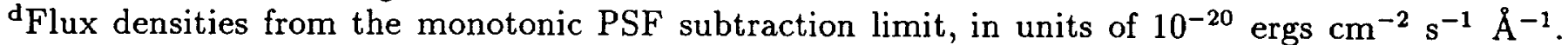
Flux densities have been corrected for galactic extinction, and normalized to $z=1$.

eFlux densities from the $\chi^{2}$ minimum PSF subtraction limit, in units of $10^{-20} \mathrm{ergs} \mathrm{cm}^{-2} \mathrm{~s}^{-1} \AA^{-1}$. Flux densities have been corrected for galactic extinction, and normalized to $z=1$.

${ }^{f}$ We note here whether nearby companions were included or not. We included all companions except the foreground spiral $c$ in the field 3C 196 and the foreground $d$ in the field of 3C 336. For those objects in which we included a nearby (perhaps discrete) companion, we give the change in flux density that would result from subtracting that companion's contribution. 


\section{TABLE 10. Radio Galaxy Near-Infrared Photometry}

\begin{tabular}{ccccc}
\hline \hline Source & Aperture $^{\mathbf{a}}$ & $F_{\lambda}$ (isophotal $^{\mathbf{b}}$ & $F_{\lambda}$ (total) $^{\mathbf{c}}$ & Notes \\
\hline \multirow{2}{*}{ 3C 175.1 } & $\mathrm{A}(0,3.5)$ & 325.7 & 447.0 & $\mathrm{~d}$ \\
& $\mathrm{~A}(0.85,3.5)$ & 128.3 & 249.6 & $\mathrm{~d}$ \\
$3 \mathrm{C} 217$ & $\mathrm{~A}(0,3.75)$ & 177.5 & 253.1 & \\
& $\mathrm{~A}(0.85,3.75)$ & 66.2 & 141.5 & \\
& $\mathrm{~A}(0,3.75)$ & 136.6 & 212.0 & $\mathrm{e}$ \\
& $\mathrm{A}(0.85,3.75)$ & 25.2 & 100.9 & $\mathrm{e}$ \\
$3 \mathrm{C} 237$ & $\mathrm{~A}(0,3.75)$ & 294.9 & 342.0 & \\
& $\mathrm{~A}(0.85,3.75)$ & 99.2 & 146.0 & \\
$3 \mathrm{C} 280$ & $\mathrm{~A}(0,3.75)$ & 598.5 & 711.2 & $\mathrm{f}$ \\
& $\mathrm{A}(0,3.75)$ & 493.6 & 605.9 & $\mathrm{fg}$ \\
& $\mathrm{A}(0.85,3.75)$ & 272.0 & 384.7 & $\mathrm{f}$ \\
& $\mathrm{A}(0.85,3.75)$ & 167.1 & 279.4 & $\mathrm{fg}$ \\
$3 \mathrm{C} 289$ & $\mathrm{~A}(0,3.75)$ & 569.0 & 735.0 & \\
& $\mathrm{~A}(0.85,3.75)$ & 422.4 & 589.0 & \\
& & & & \\
\hline \hline
\end{tabular}

${ }^{a}$ Aperture size; $\mathrm{A}(r 1, r 2)$ signifies annular aperture with inner radius $r 1$ and outer radius $r 2$, where $r 1$ and $r 2$ are in arcseconds.

${ }^{b}$ Flux densities in units of $10^{-20} \mathrm{ergs} \mathrm{cm}^{-2} \mathrm{~s}^{-1} \AA^{-1}$, above an isophotal cutoff level of $4.56 \times 10^{-19} \mathrm{ergs} \mathrm{cm}^{-2} \mathrm{~s}^{-1} \AA^{-1} \mathrm{arcsec}^{-2}$, normalized to $\mathrm{E}(B-V)=0, z=1$.

'Total flux densities in aperture, with no isophotal cutoff, in units of $10^{-20}$ ergs cm-2 $\mathrm{s}^{-1} \AA^{-1}$. Flux densities have been corrected for galactic extinction and normalized to $z=1$.

${ }^{d}$ Foreground star removed. Companions to NE included; their contribution to $F_{\lambda}($ total $)=30$, and to $F_{\lambda}($ isophotal $)=6$.

${ }^{\circ}$ Components $a$ and $c$ excluded.

${ }^{\mathrm{f}}$ Foreground galaxy removed.

${ }^{\mathrm{g}}$ Companions, eastern lobe region excluded. 


\section{PSF Subtraction Tests}

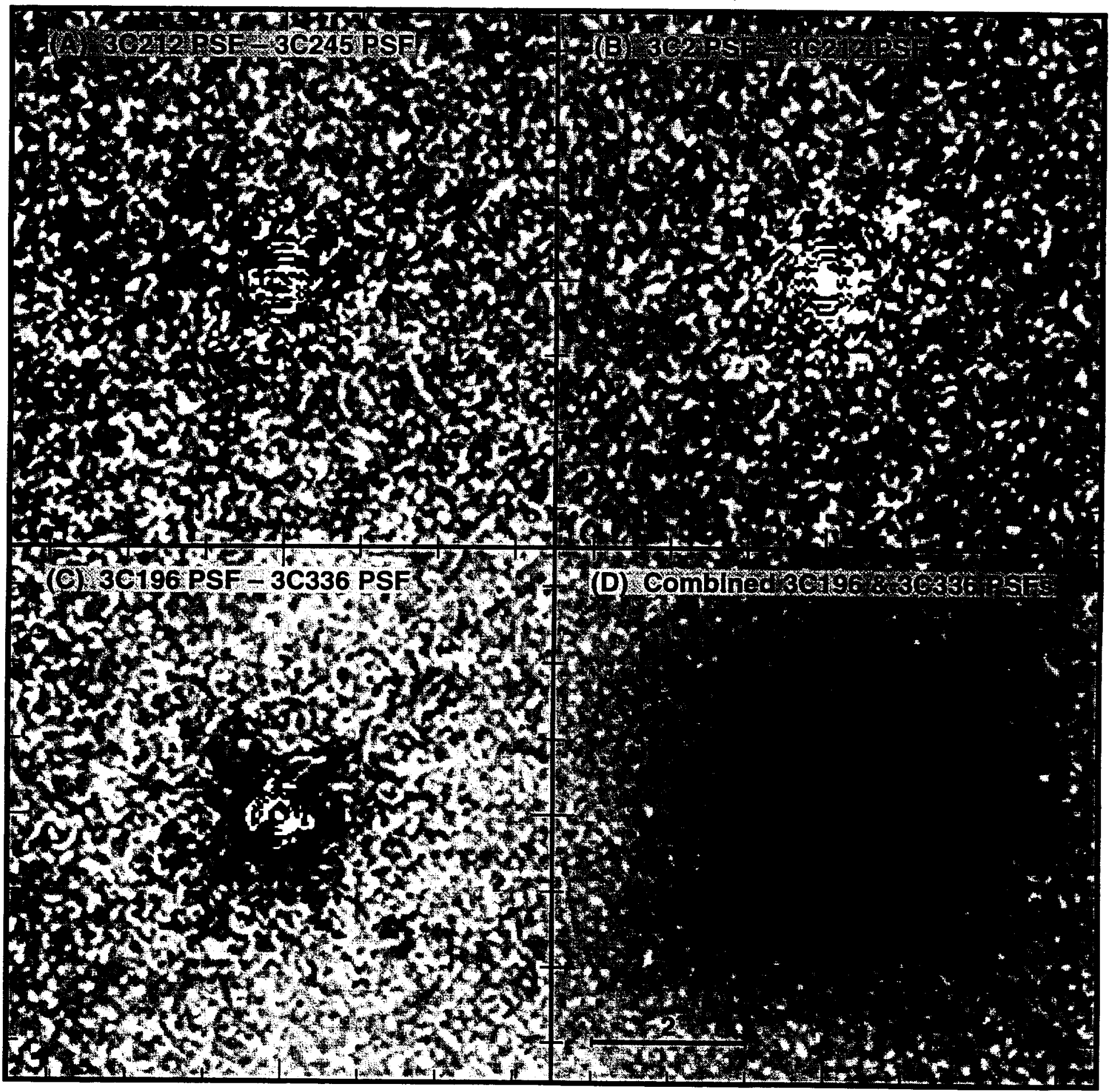




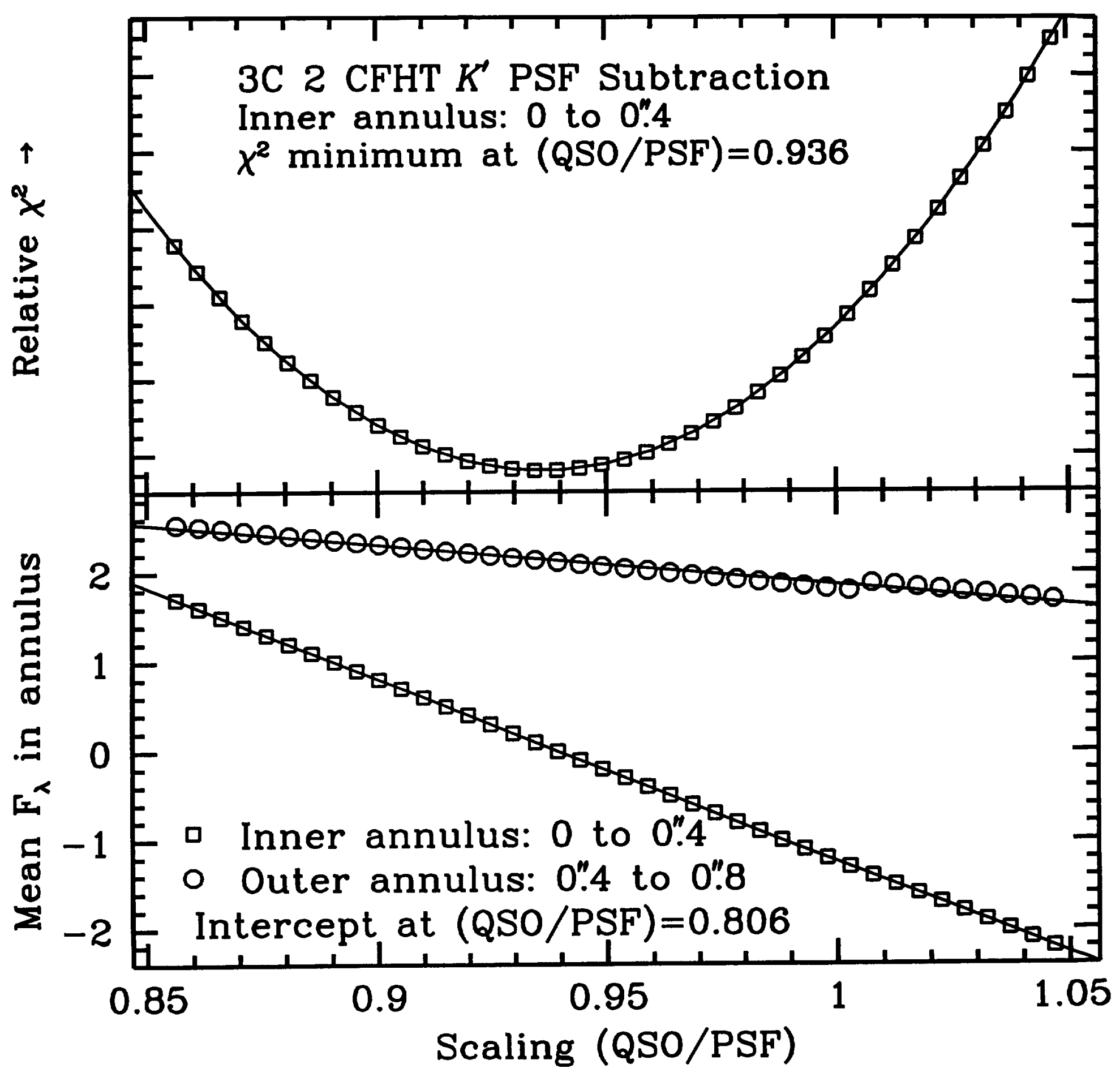


3C 2

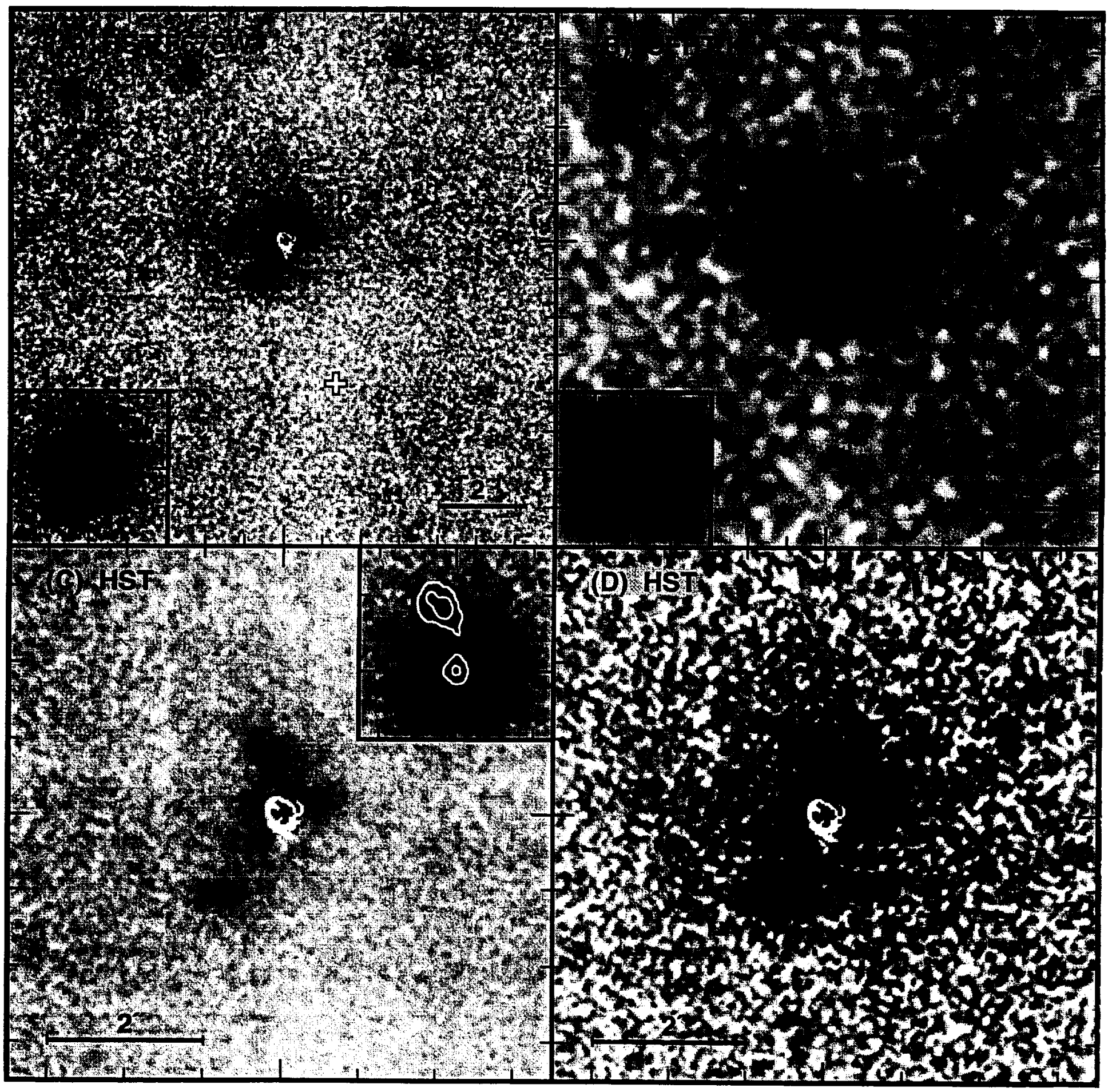




\section{C 175.1}

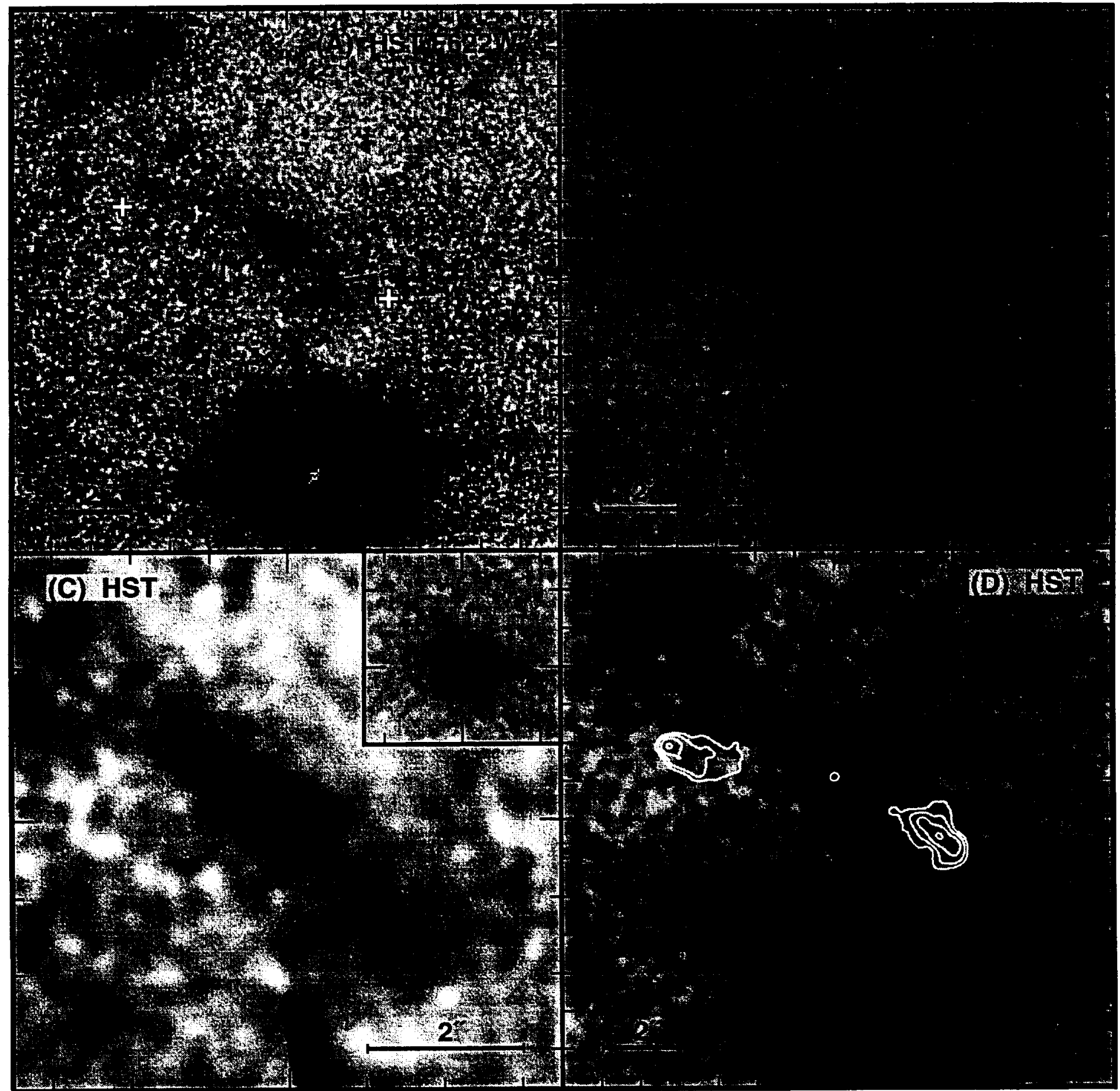




\section{C 196}

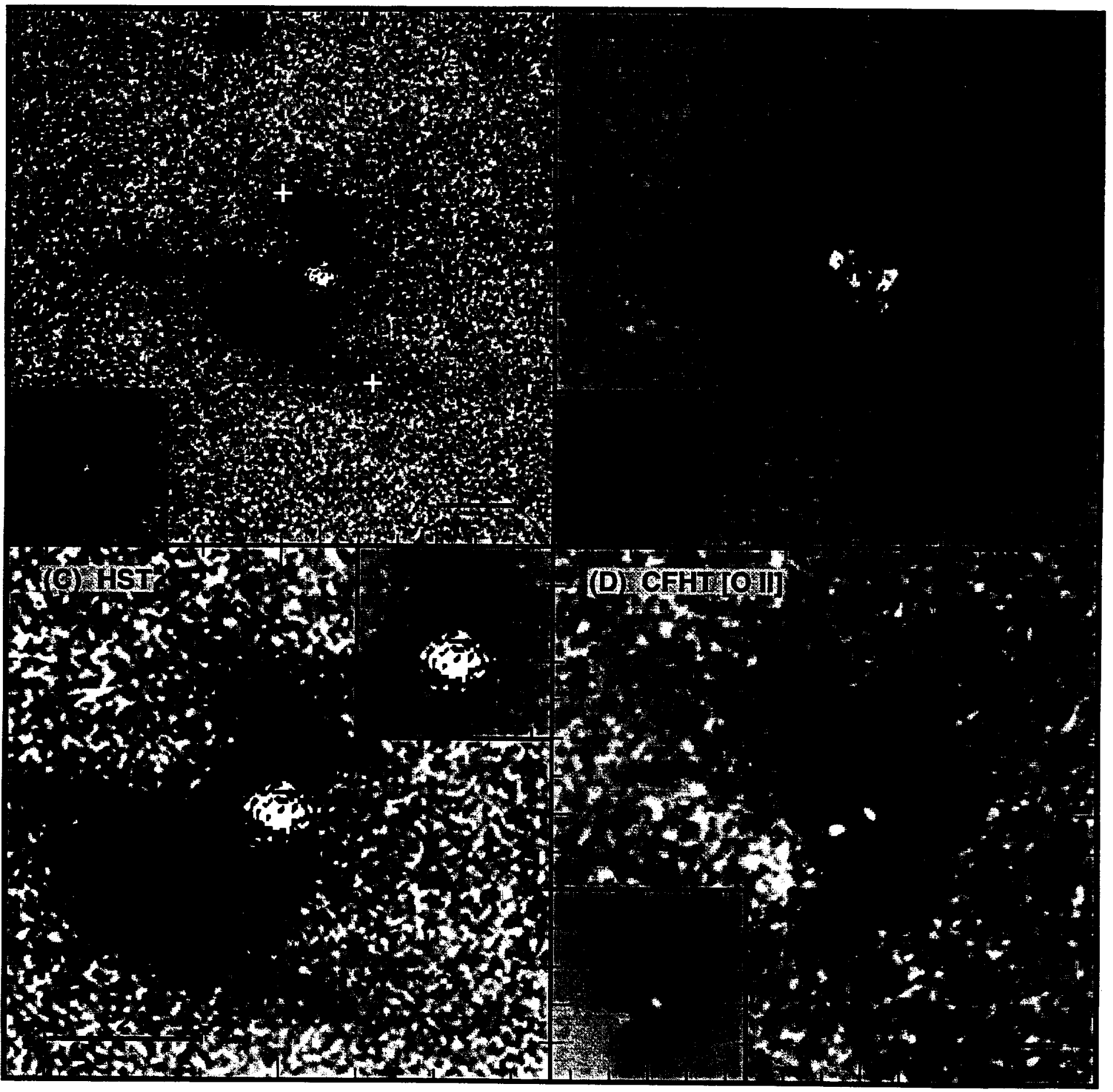


3C 212

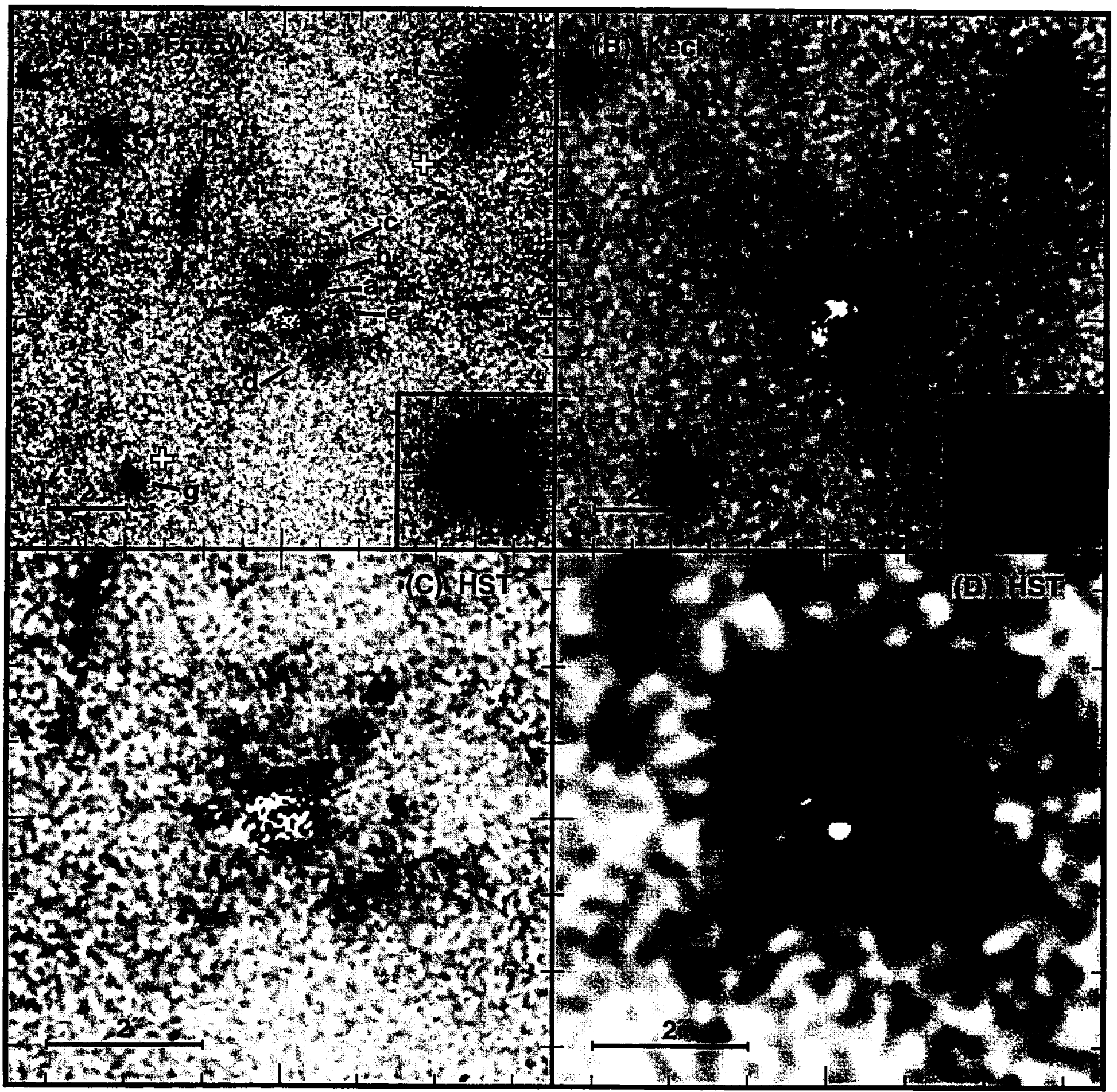




\section{C 217}

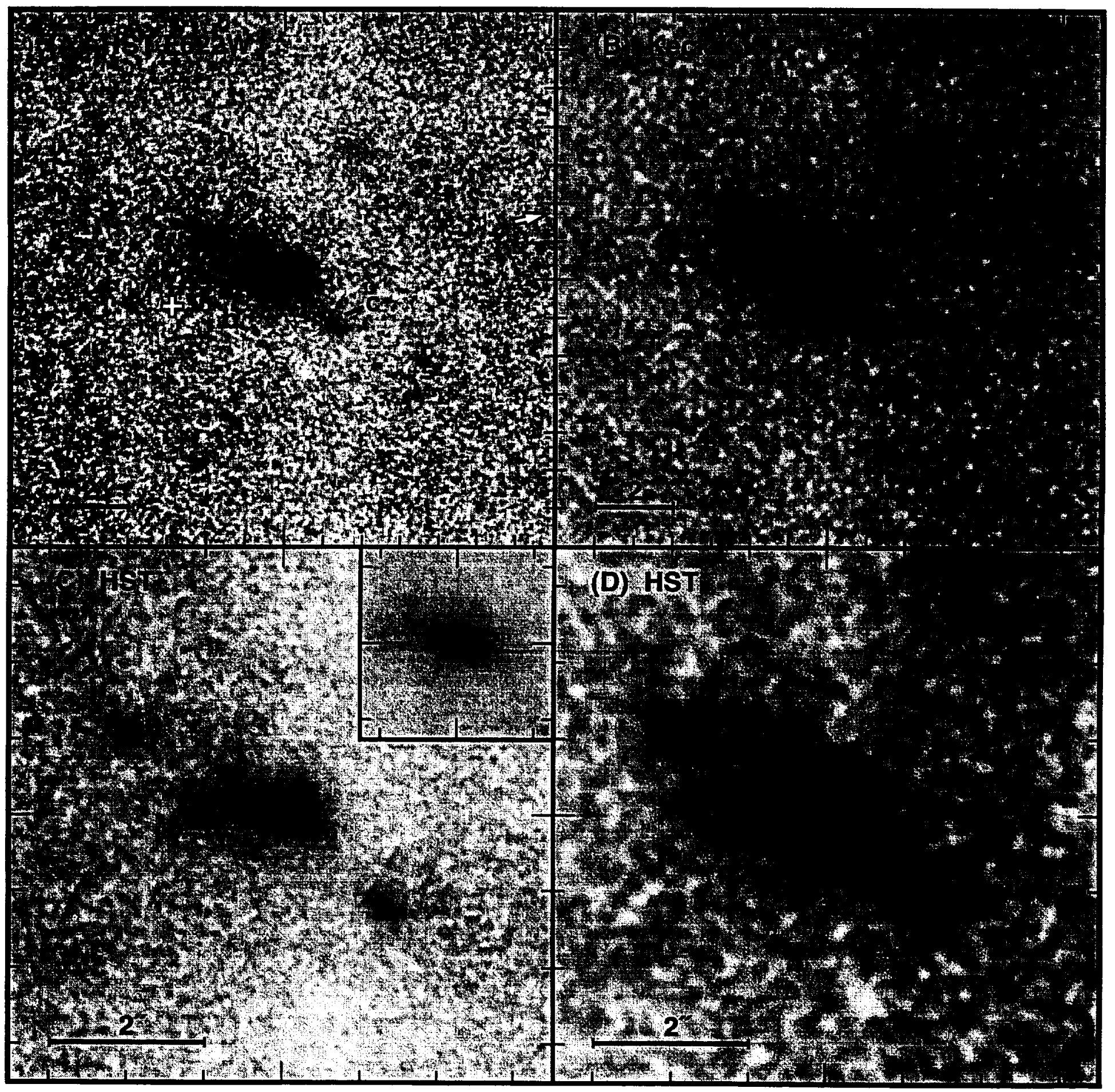




\section{C 237}

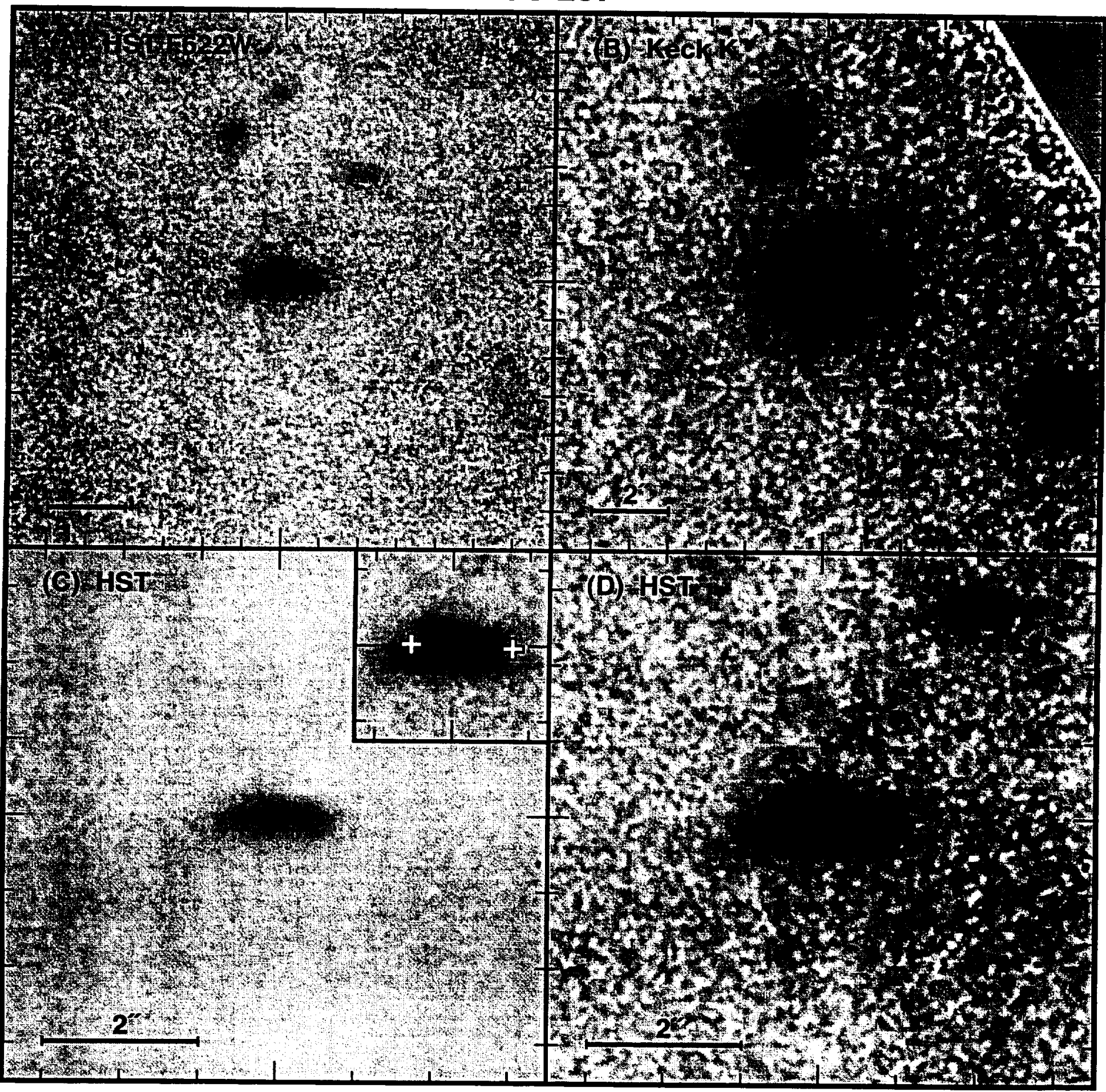




\section{C 245}

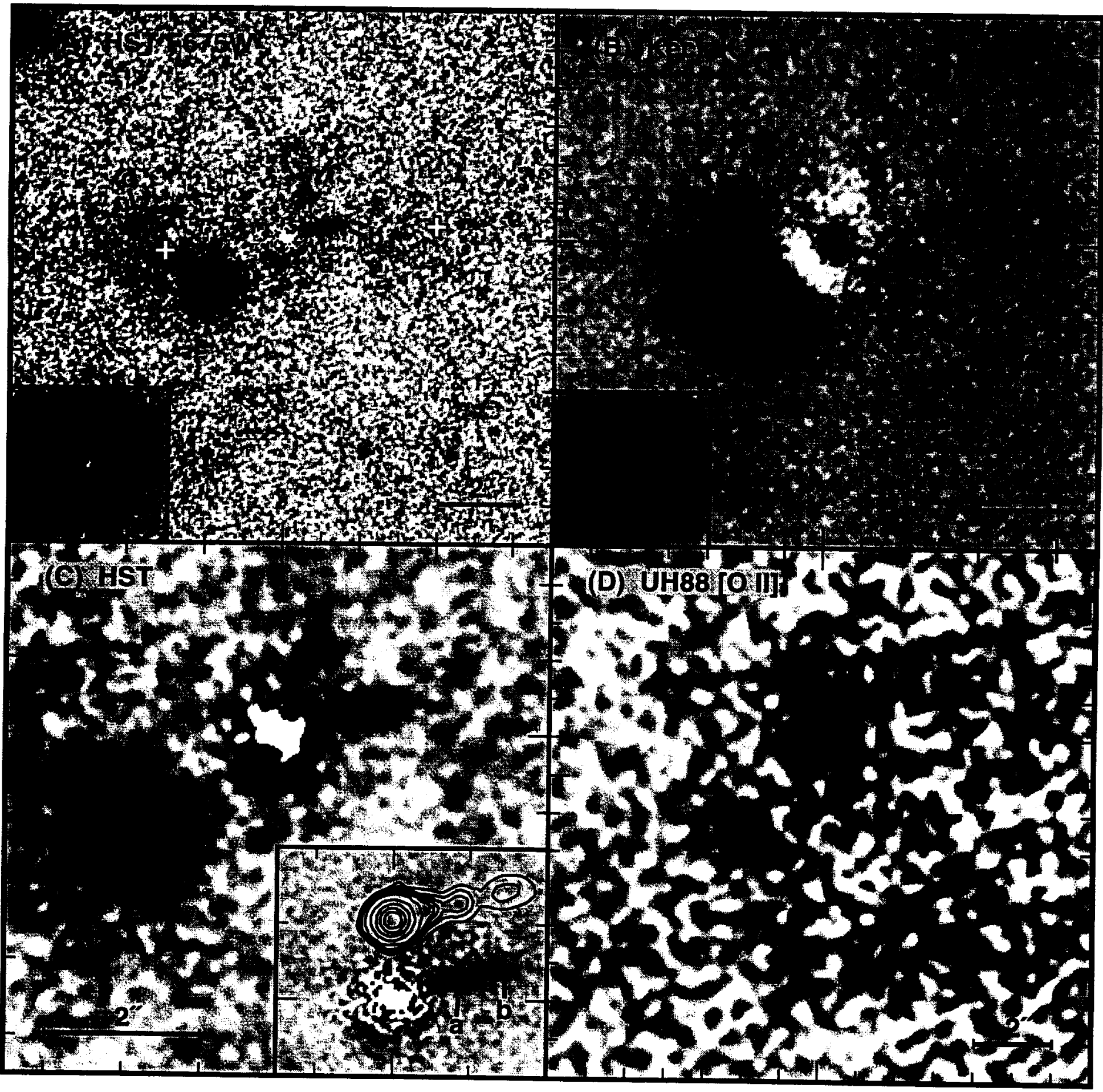


3C 280

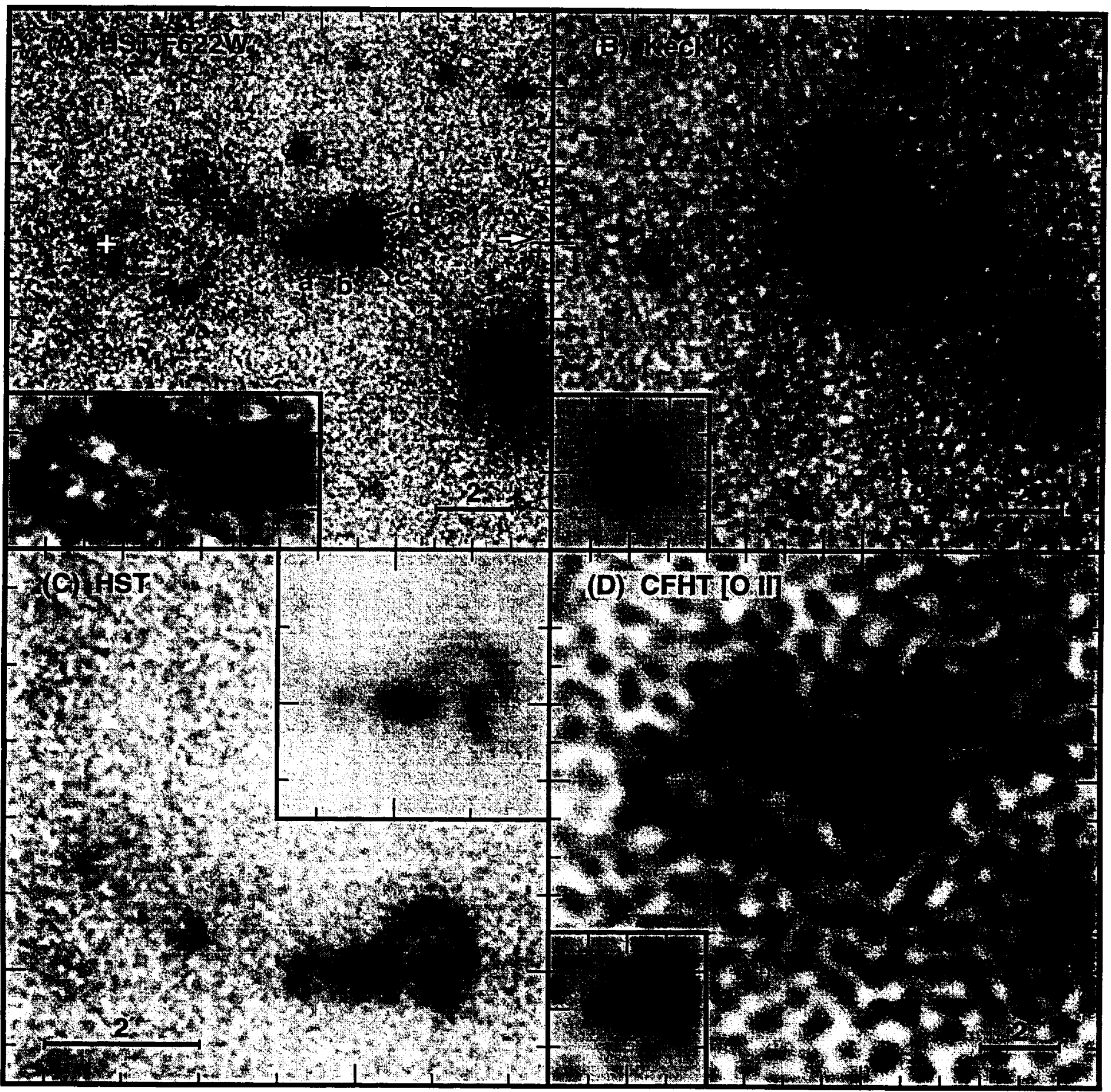




\section{C 289}

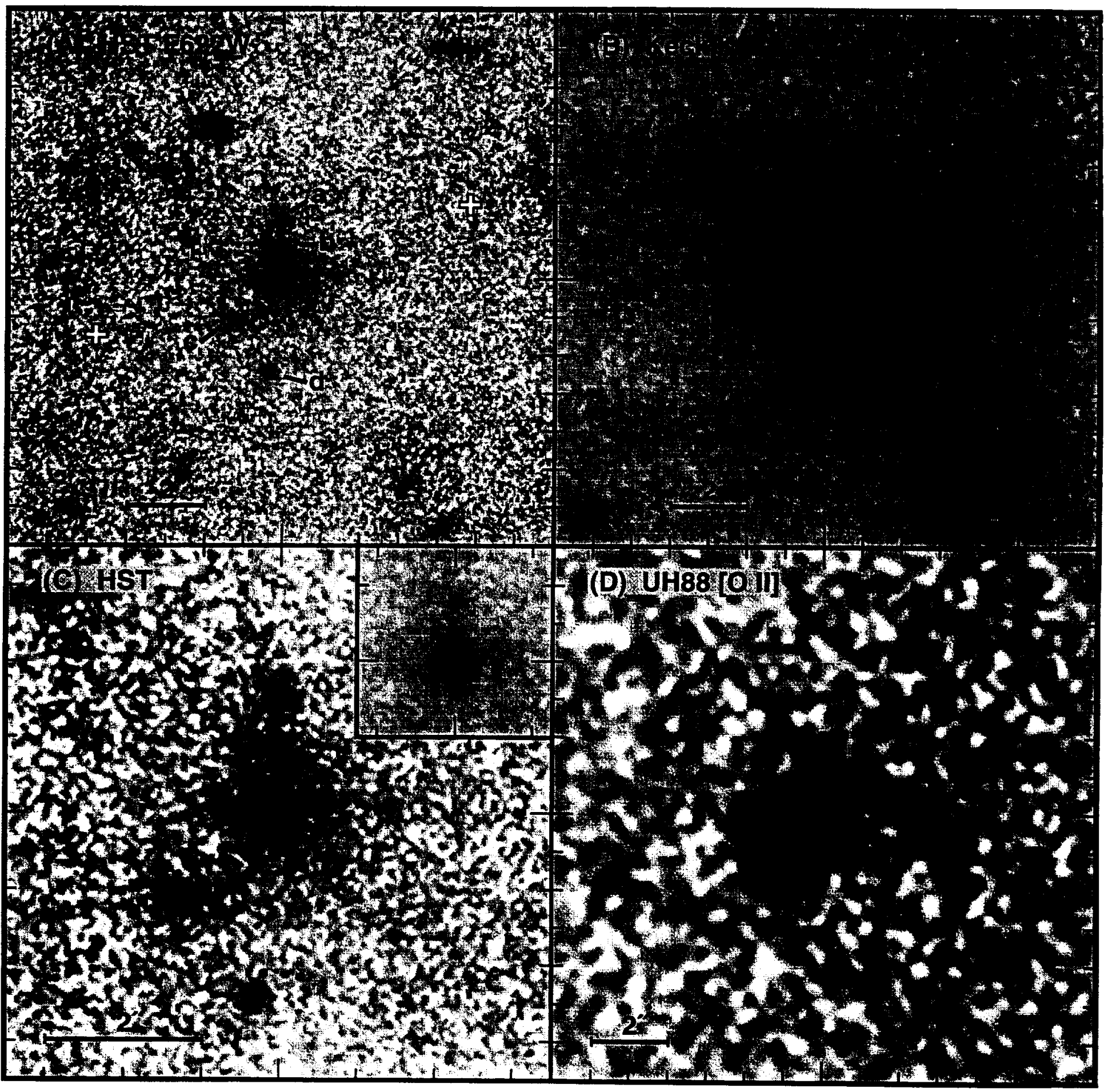




\section{C 336}

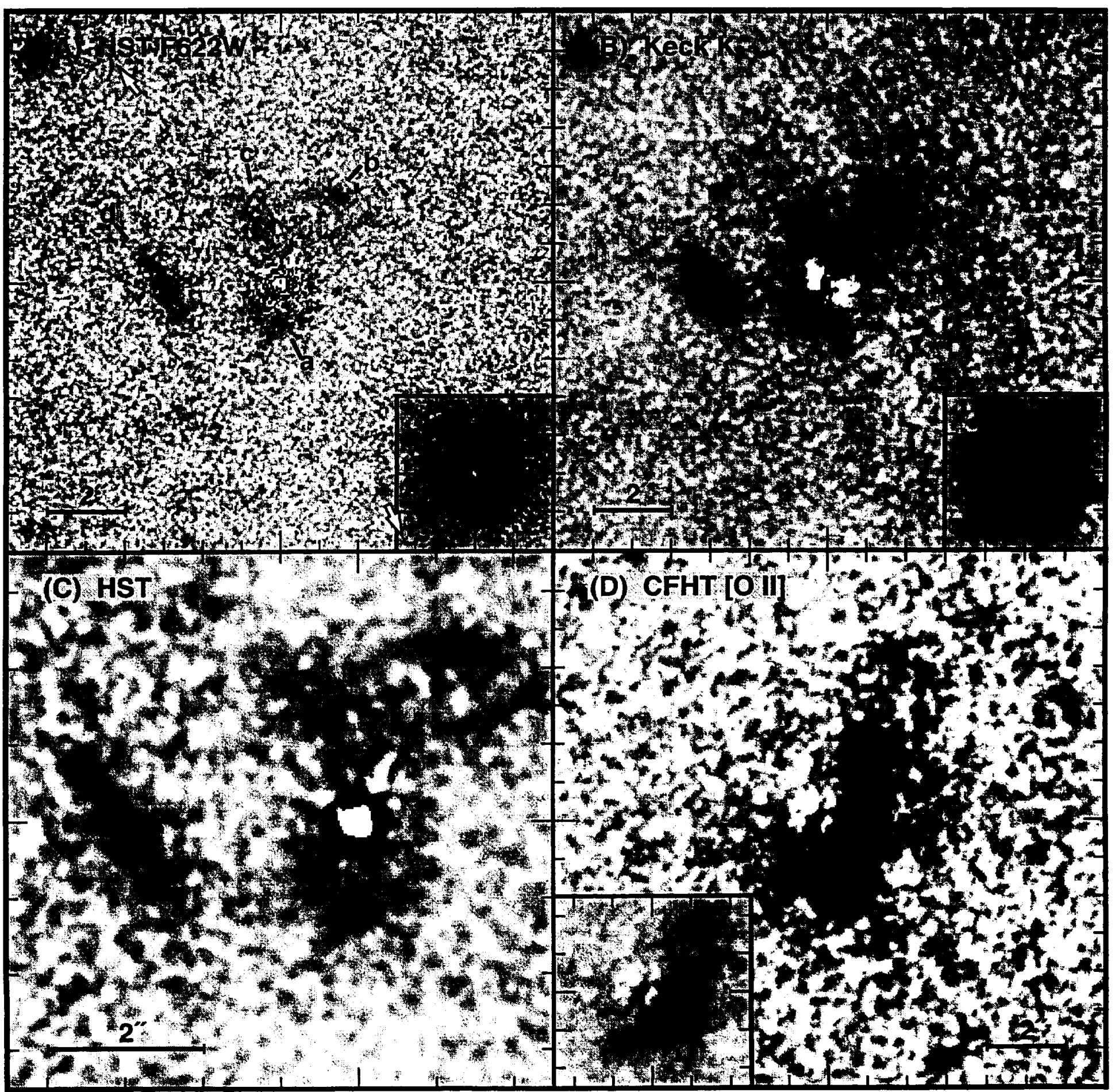




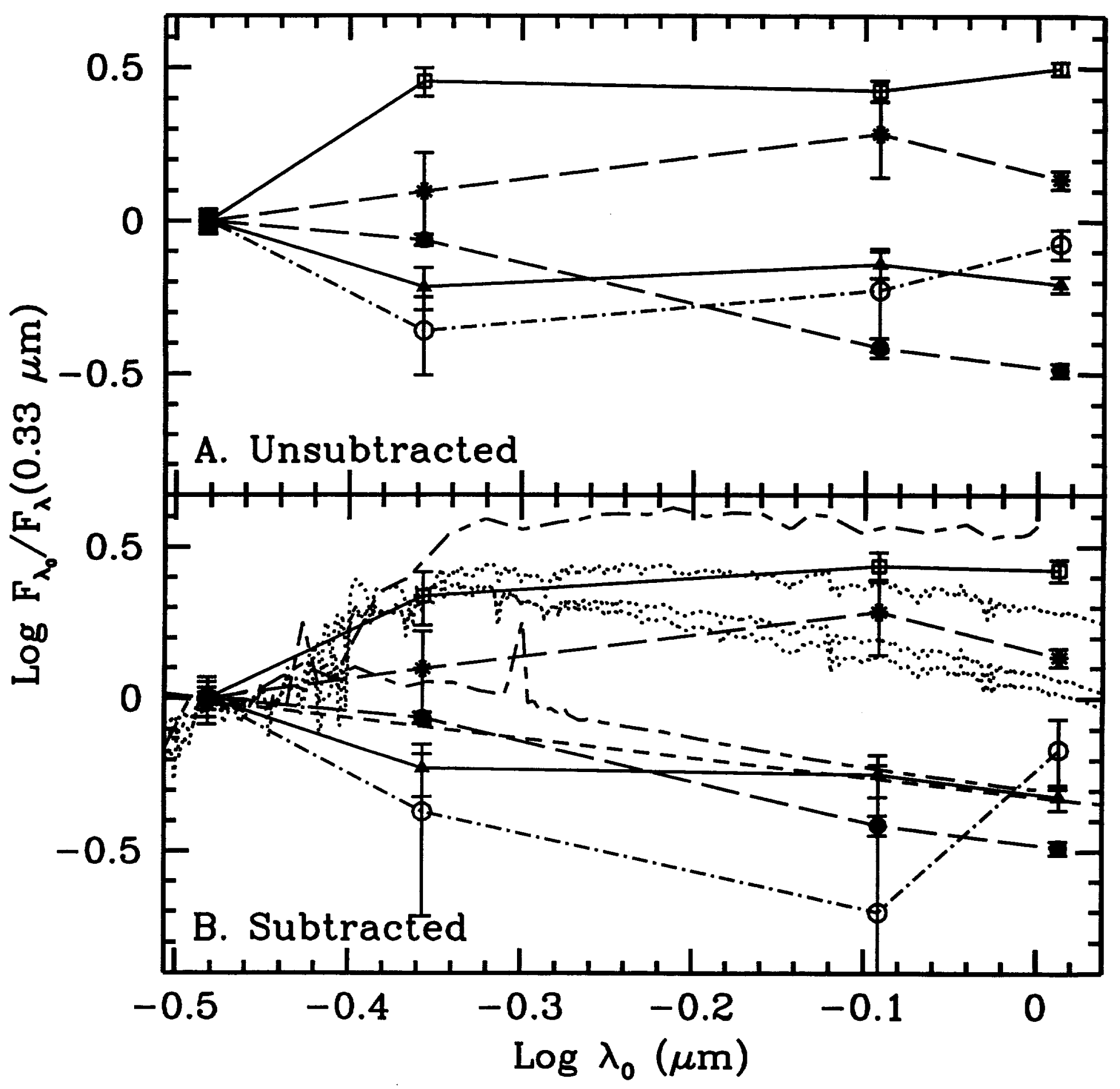




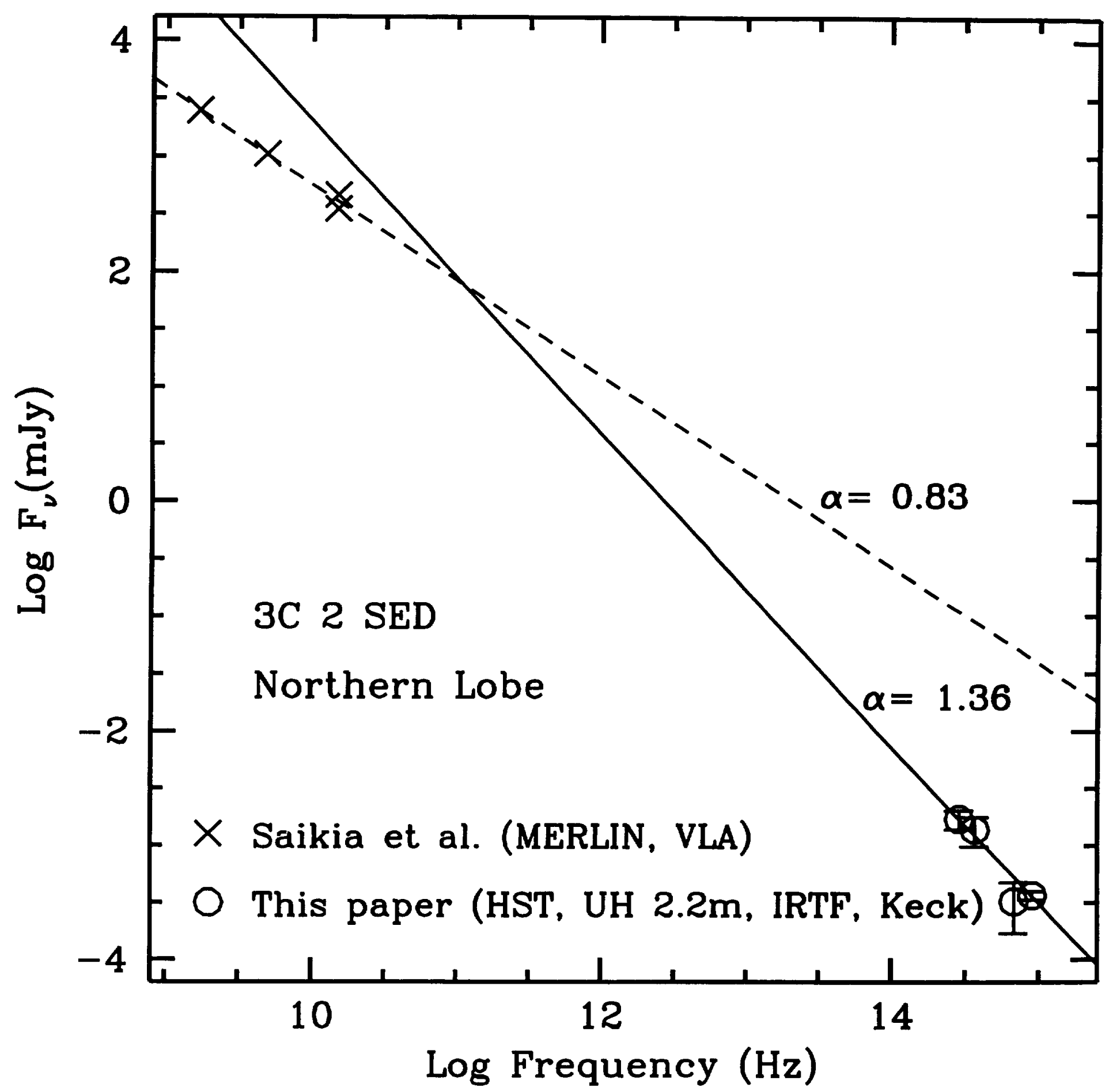




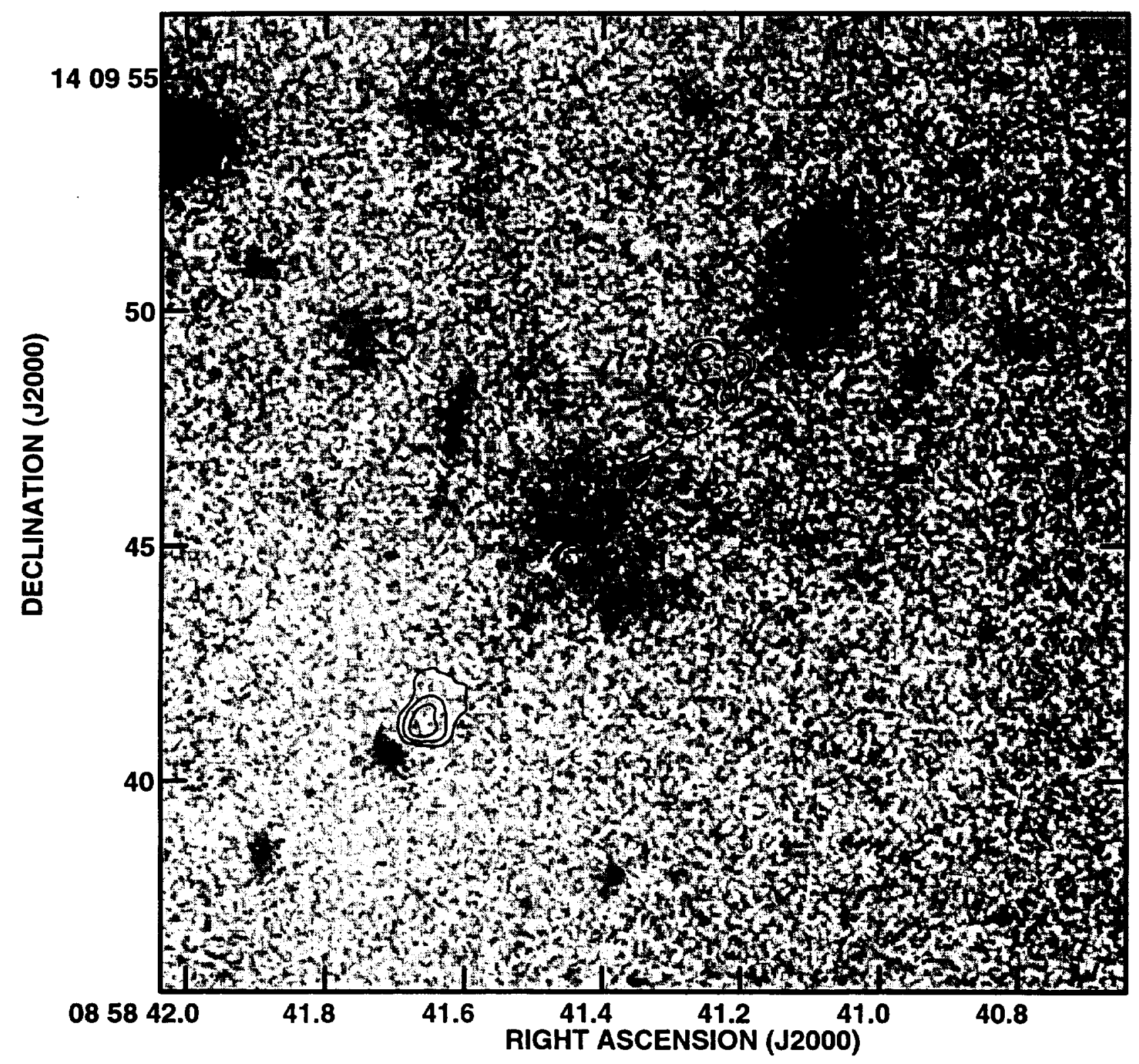




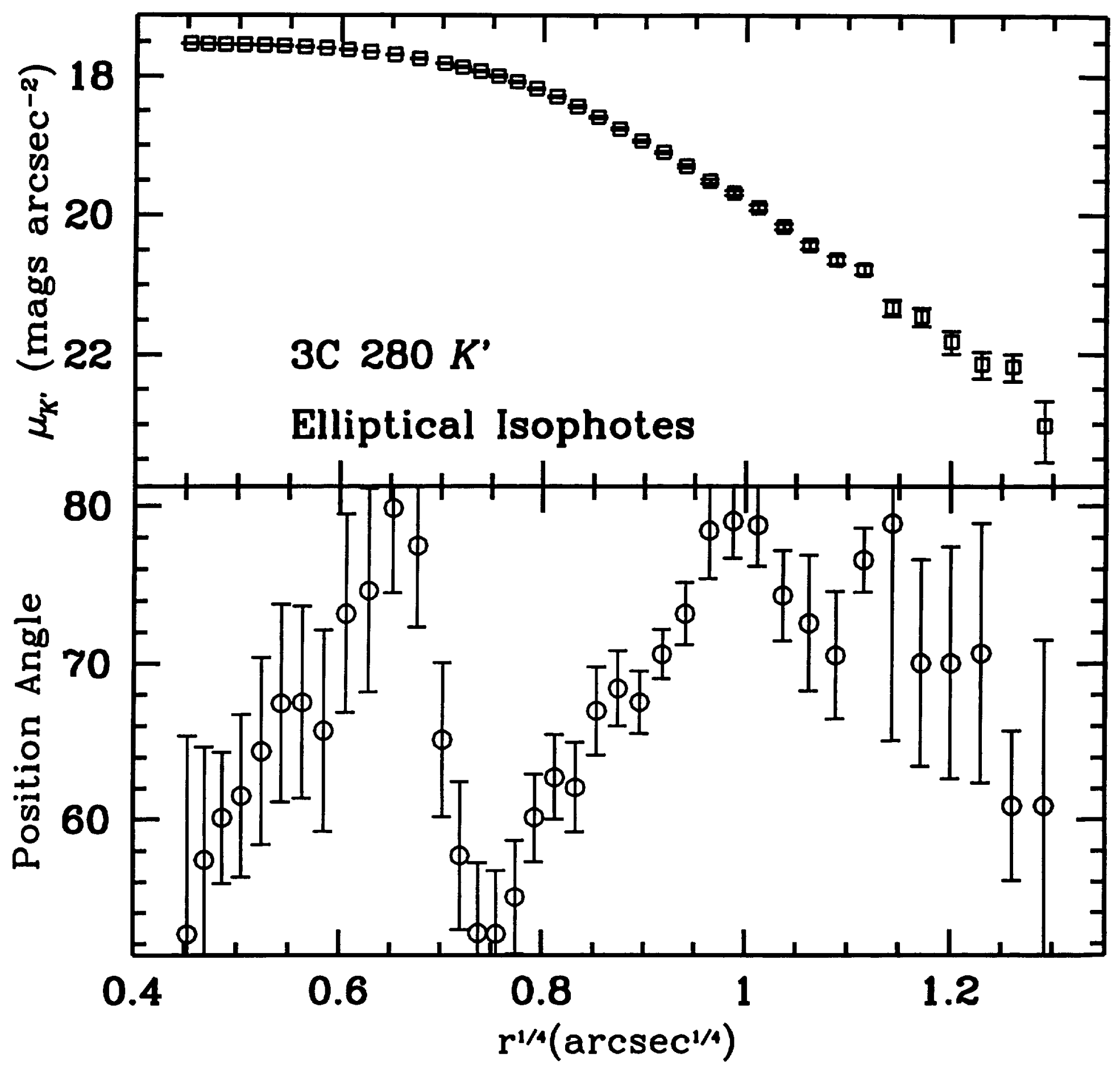

Figure 16 


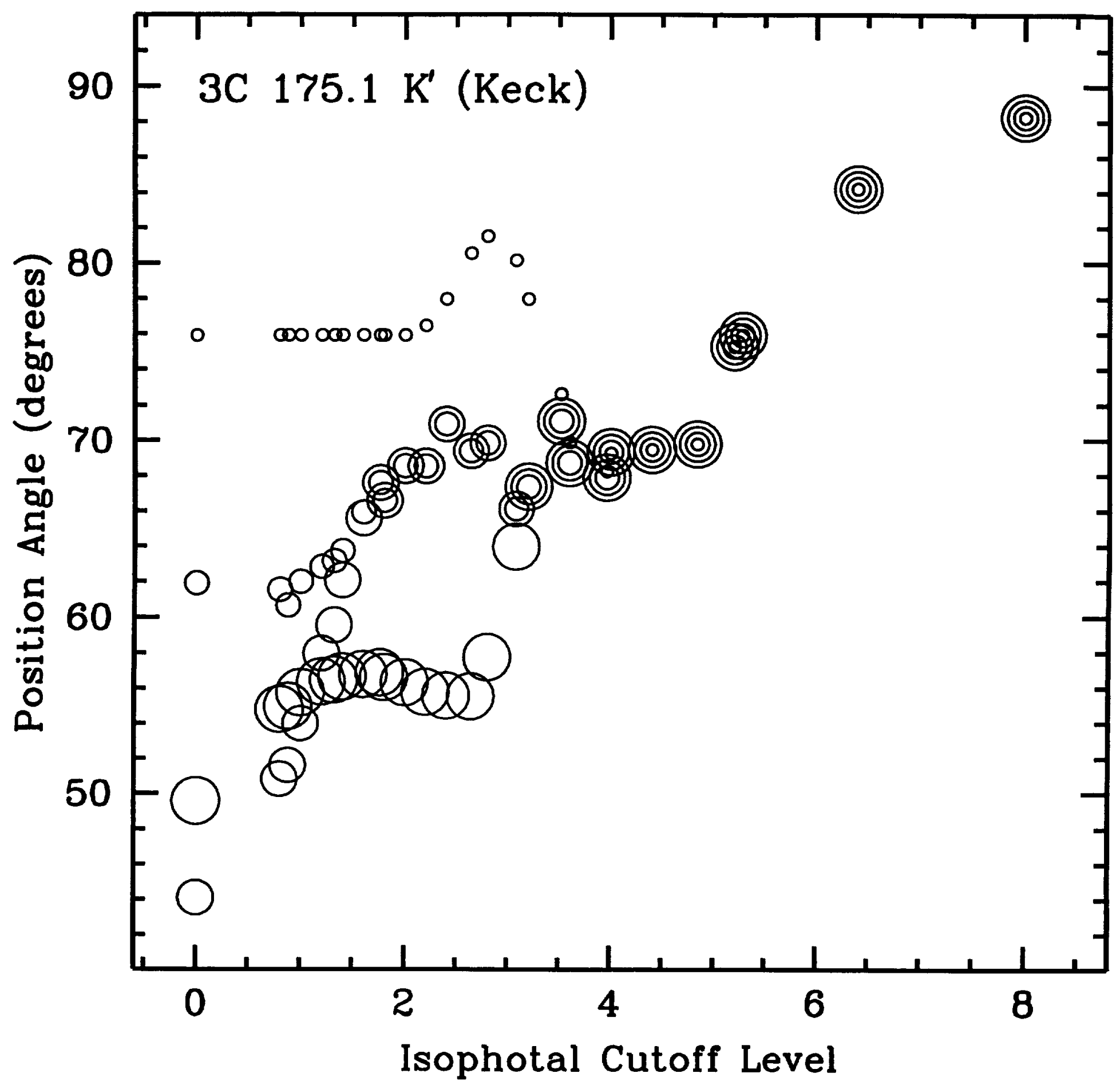




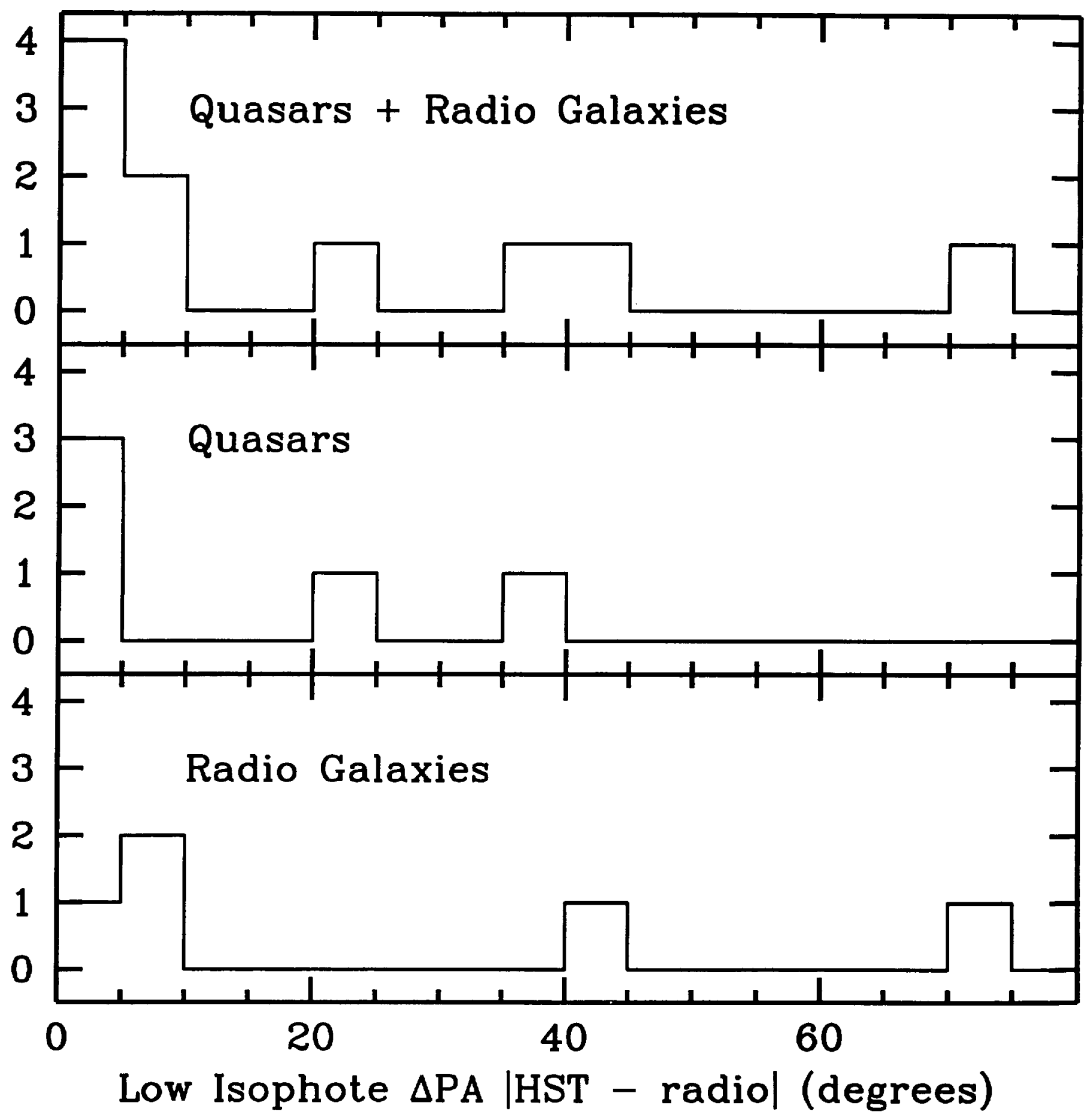

Figure 18 


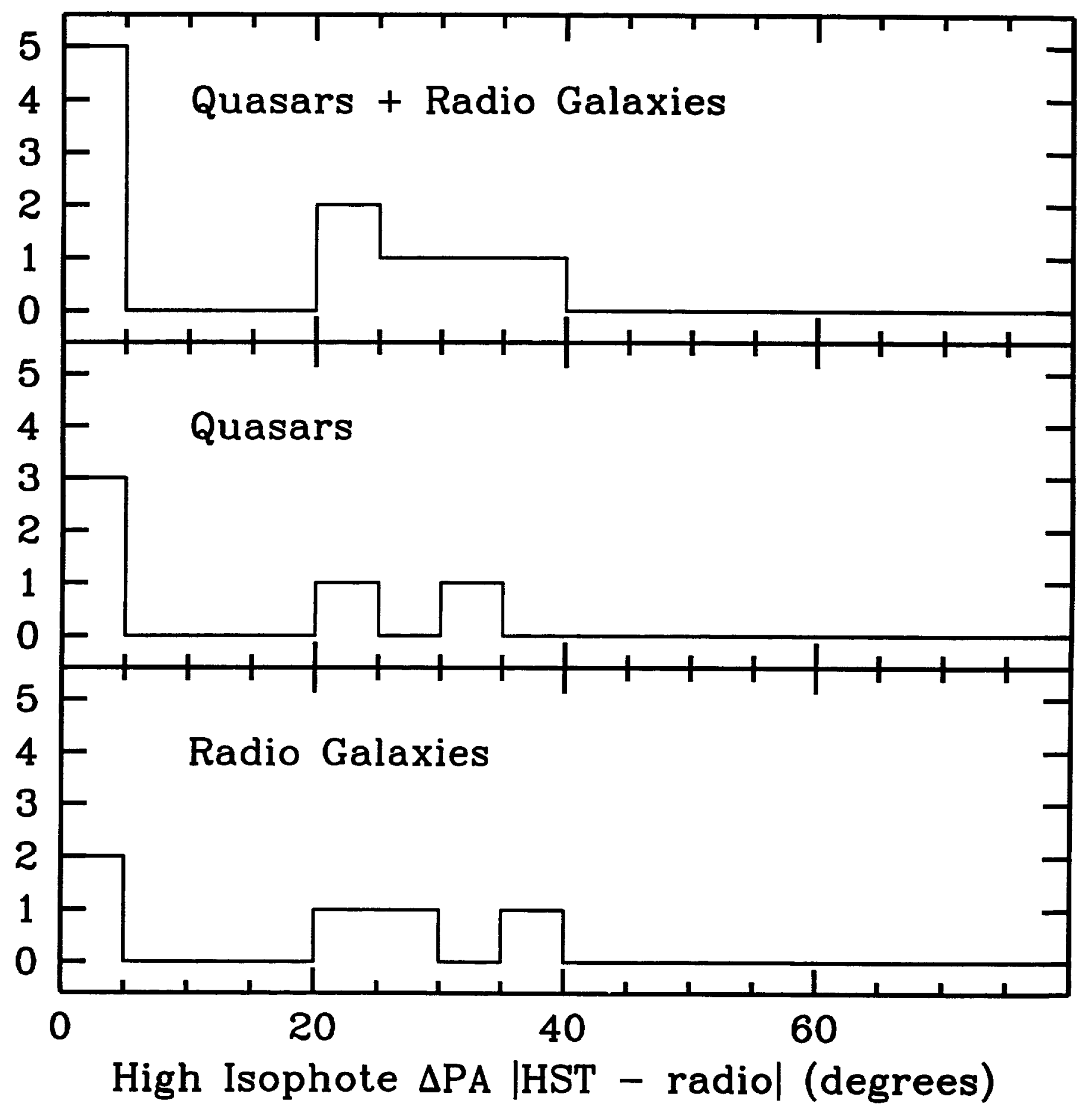




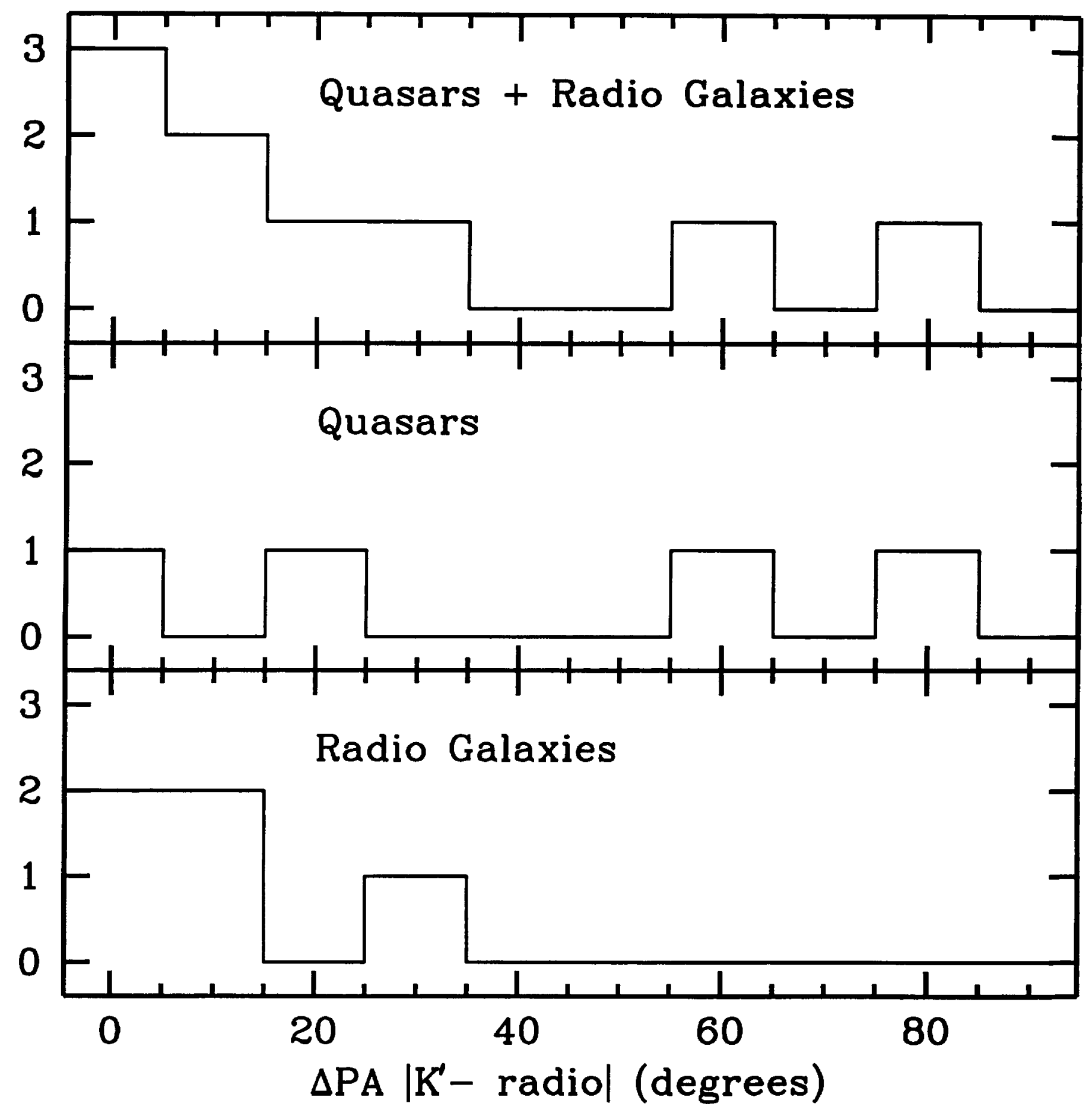




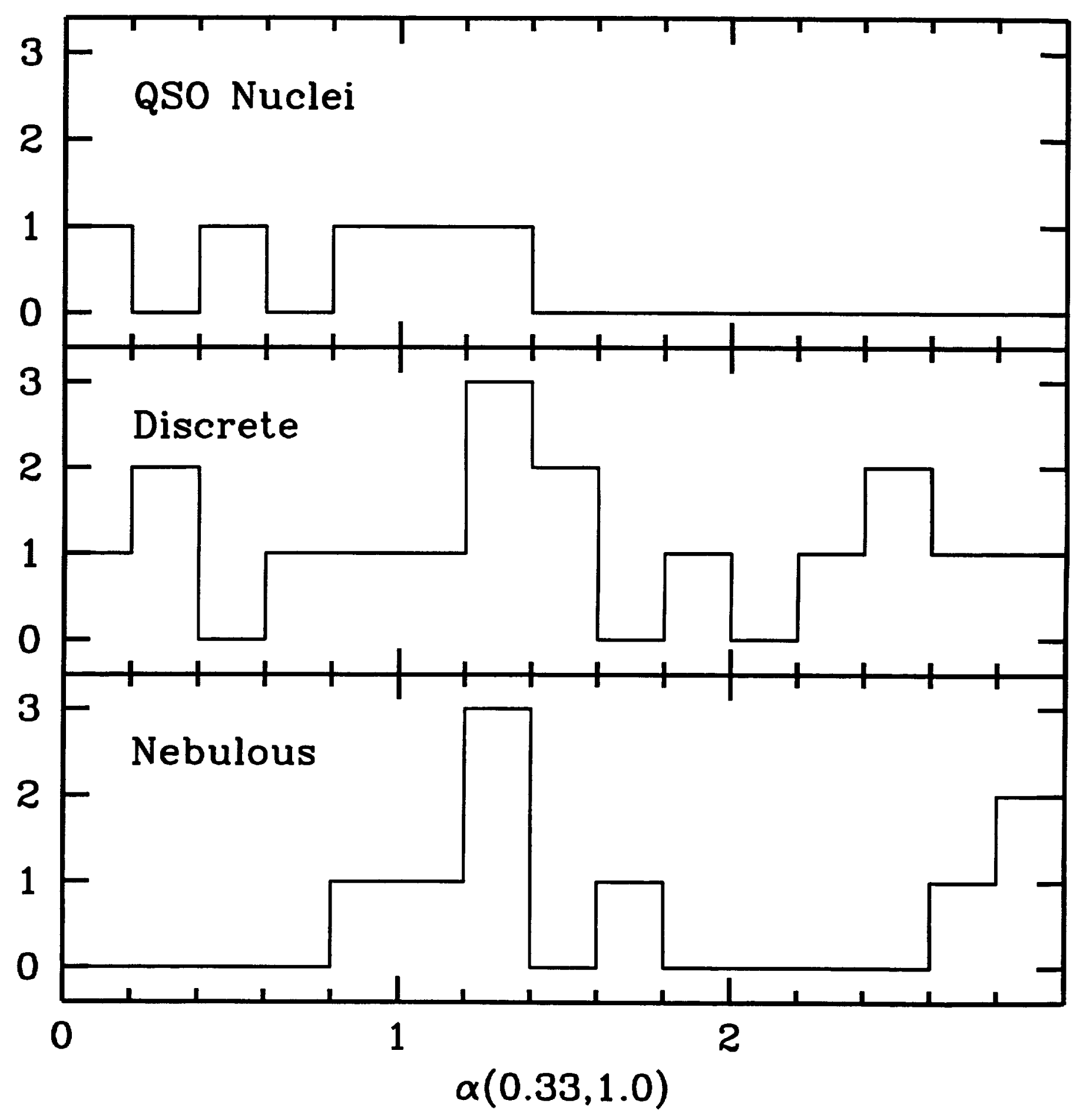




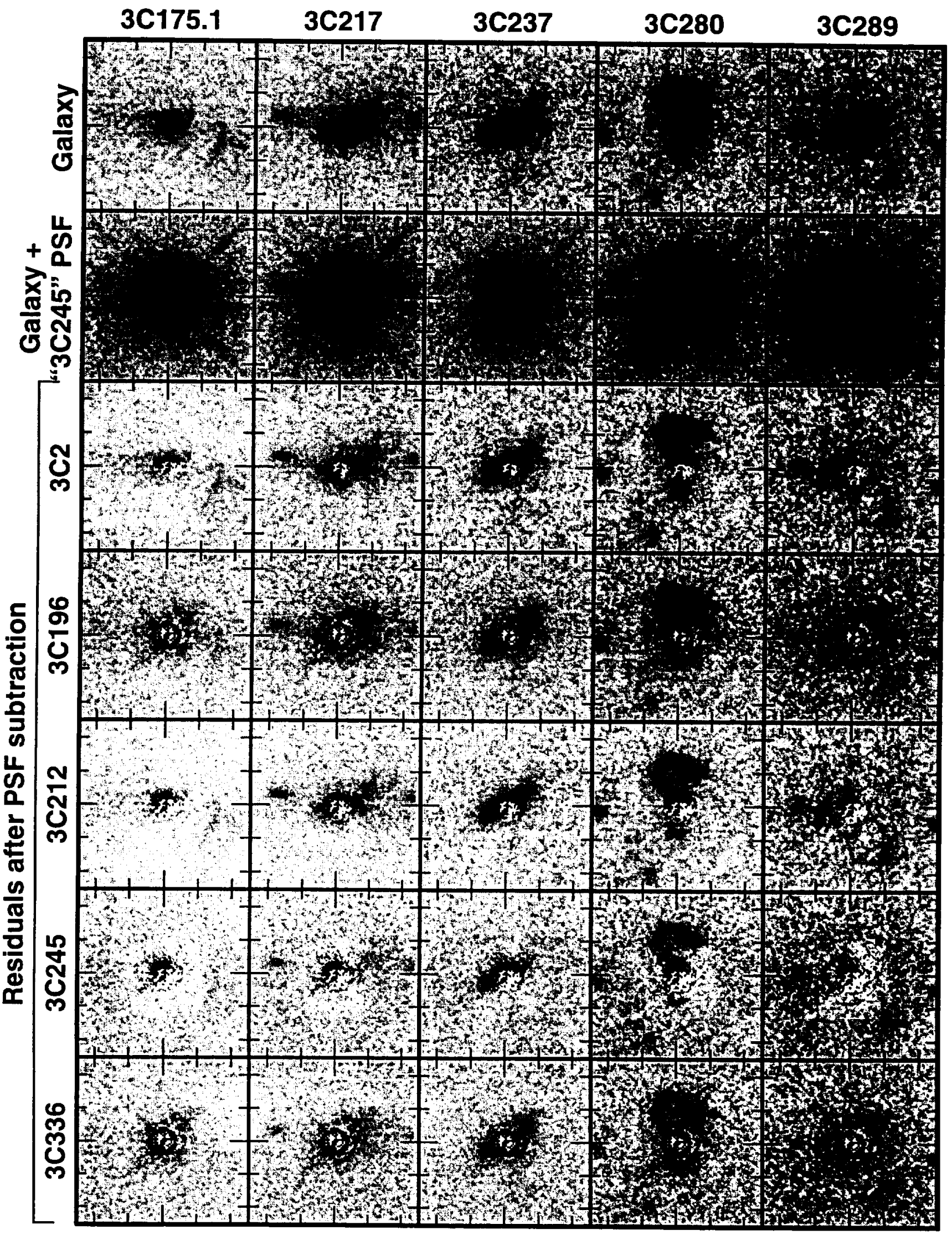


